ESAIM: M2AN 49 (2015) 875-919

DOI: $10.1051 / \mathrm{m} 2 \mathrm{an} / 2014061$
ESAIM: Mathematical Modelling and Numerical Analysis

www.esaim-m2an.org

\title{
SMALL-TIME SOLVABILITY OF PRIMITIVE EQUATIONS FOR THE OCEAN WITH SPATIALLY-VARYING VERTICAL MIXING
}

\author{
HiROTADA HONDA ${ }^{1}$
}

\begin{abstract}
The small-time existence of a strong solution to the free surface problem of primitive equations for the ocean with variable turbulent viscosity terms is shown in this paper. In this model, the turbulent viscosity coefficients, which include the Richardson number depending on unknown variables, are explicitly formulated. In addition, following the formulation of practical models, the kinematic condition is assumed on the free ocean surface. As in preceding works, we consider the problem in the three-dimensional strip-like region, and assume the $f$-approximation. Under some conditions on the initial and boundary data and the topography of the bottom of the ocean, we construct a strong local-in-time solution in Sobolev-Slobodetskil spaces. The boundedness of the temperature and salinity is also shown in the present paper.
\end{abstract}

Mathematics Subject Classification. 35M10, 35Q35, 35R35.

Received March 1st, 2012. Revised September 6, 2014.

Published online April 16, 2015.

\section{INTRODUCTION}

In the present paper, we investigate a free surface problem of primitive equations for the ocean while taking vertical mixing into account, and show the unique existence of a strong local-in-time solution, which is a new result developed from our earlier results [23-26].

Since the memorable contributions to the mathematical argument of primitive equations by Lions et al. [33,34], there have been a number of works concerning primitive equations for the ocean, the atmosphere, and the coupled model of the ocean and the atmosphere [35-39] in the mathematical literature. Here we summarize a part of these, with a particular focus on the ocean model.

As for weak solutions in an ocean domain without sidewalls, we have had two results to date. Azerad and Guillén-González [1] discussed the non-stationary, and Besson and Laydi [5] the stationary case. In [1], they showed the existence of a weak solution of the Navier-Stokes equations with anisotropic viscosity terms and its convergence with a weak solution of primitive equations, as the aspect ratio of the depth to width of the domain tends to zero. As far as we know, no contributions have been made with results concerning strong solutions in this type of domain.

For two-dimensional primitive equations, we have some results concerning a strong global-in-time solution. Guillén-González and Rodríguez-Bellido [19] showed the existence and uniqueness of the strong solution to

\footnotetext{
Keywords and phrases. Primitive equations, Sobolev-Slobodetskiǔ space, strong solution.

1 Department of Mathematics, Keio University, 3-14-1 Hiyoshi, 223-8522 Yokohama, Japan. t.asc2904@gmail.com
} 
primitive equations for the ocean in the two-dimensional region with a sidewall under the smallness of data. Bresch et al. [8] also showed the existence and uniqueness of a strong solution with large data, and also showed the uniqueness of the weak solution in a similar region. Hu et al. [27] proved the existence and uniqueness of a strong solution to the primitive equations on thin domains with a non-flat bottom. Following that, Hu [28] discussed primitive equations for the ocean under a small depth assumption, and proved that the solution is represented asymptotically as a sum of barotropic flow both in the horizontal and vertical direction as $\epsilon$ goes to zero. However, in these cases, Coriolis force does not make sense.

Concerning a strong solution of primitive equations for the ocean in the three-dimensional region, we largely have two types of results in past arguments, with and without sidewalls (the vertically flat lateral boundary). Guillén-González et al. [20,21] discussed the initial boundary value problem for primitive equations for the ocean in the domain surrounded by a rigid lid, sidewalls and bottom. They showed the existence of a global strong solution with small data and a local strong solution with any data in $L^{\infty}\left(0, T ; H^{1}(\Omega)\right) \bigcap L_{2}\left(0, T ; H^{2}(\Omega)\right) \cap W_{2}^{1}\left(0, T ; L_{2}(\Omega)\right)$. In a similar situation, Temam and Ziane [51] verified the existence and uniqueness of a strong local-in-time solution of primitive equations for the ocean in $C\left(0, T ; H^{1}(\Omega)\right) \cap L_{2}\left(0, T ; H^{2}(\Omega)\right)$. Their results are based on Ziane's preceding results $[55,56]$ concerning the elliptic problem in a domain with corners. Later, Cao and Titi [11] showed the existence and uniqueness of a global solution in $C\left(0, T ; H^{1}(\Omega)\right) \bigcap L_{2}\left(0, T ; H^{2}(\Omega)\right) \bigcap W_{1}^{1}\left(0, T ; L_{2}(\Omega)\right)$. As far as we know, however, a formulation with a free ocean surface, which is frequently used in the practical model, has not been discussed in the mathematical literature.

We will now summarize past arguments in weather prediction and oceanography. Following the proposals of Bjerknes [4] and the pioneering numerical modeling of the atmosphere by Richardson [43], Bryan's work [9] was the first to model ocean circulation, which applied the rigid lid hypothesis, that is, the ocean surface was assumed to be flat and fixed. This is because they preferred to remove the effect of gravitational waves of low frequencies, in order to take long as an interval length of numerical integration as possible. However, the barotropic component in ocean movement was not removed, which requires an additional calculation for the stream function, called the relaxation method [30]. Based upon his formulation Semtner [44] proposed the general circulation model and numerically studied it in detail. In his model, the Boussinesq approximation and the rigid lid hypothesis were used. After that, a trial of the free ocean surface model followed. Crowley $[13,14]$ was the first person that conceived the free ocean surface model for numerical calculation. In that model, integration with respect to the vertical coordinate system was introduced, and the problem was reduced to that in the two-dimensional framework. Later, Blumberg and Mellor [7], Dukowicz and Smith [15], and Killworth [30] proposed the free surface model modifying those developed by Bryan [9] and Cox [12]. For the rationale of the model by Killworth, see [30] and the references therein ([6], for example). Combining these models, the modular ocean model (MOM) $[40,41]$ became one of the most popular methods for modeling of the ocean. Although a number of model options be selected, it includes the free ocean surface model as a default.

In a series of our works [23-26], we considered the free surface problems of primitive equations. There, we formulated the original problem in the Cartesian coordinate first, and then transformed it into the $p$-coordinate system by making use of the hydrostatic relationship $\partial p / \partial x_{3}=-\varrho g$. Then, we also made the problem into that in the known fixed region by another coordinate transform in order to consider the coupled model. The kinematic condition $\frac{\mathrm{D}}{\mathrm{D} t} F(x, t)=0$, with $F$ the graph of the free surface, was not assumed. This is because we rigorously consider the effect of evaporation and condensation, while the kinematic condition assumes that a particle on the ocean surface adheres to it. In addition, the effect of ocean surface tension was taken into account in the boundary condition on the free ocean surface, which was the original one in the literature on the mathematical analysis of primitive equations. Another feature in our preceding models is the stress tensor in the conditions on the ocean surface, which is usually not considered in atmospheric science and oceanography. On the other hand, in the practical models, some empirical formulations such as the kinematic condition and the bulk formulae for the flux of momentum and heat, are applied in the conditions on the ocean surface. The effect of ocean surface tension is not taken into account even in the free ocean surface model. Nevertheless, since 
there are no works concerning these models in the mathematical literature, it is uncertain if the practical model is mathematically well-posed.

In the present paper, we discuss the ocean model with boundary conditions subject to MOM3 [40] and MOM4 [41]. The kinematic condition, which our preceding works did not adopt, was applied in this paper. We do not apply a $p$-coordinate system or the Lagrangian coordinate system in this paper.

The second feature of the present work is taking the parameterization of vertical mixing into account. All of the existing results in the mathematical literature were found by regarding the turbulent viscosity and diffusivity coefficients as positive constants, while it is known that modeling them as functions of the Richardson number sometimes yields better suitability $[29,31,42]$. Mathematically, this requires a higher regularity of the obtained velocity under the appropriate assumptions of the regularity of data. Following Pacanowski and Philander [42], and Washington and Parkinson [53], the representation of the Richardson number in this paper is provided as $\mathcal{R}\left(\frac{\partial \mathbf{v}}{\partial x_{3}}, \varrho, \frac{\partial \varrho}{\partial x_{3}}\right)=g \varrho^{-1} \frac{\partial \varrho}{\partial x_{3}}\left|\frac{\partial \mathbf{v}}{\partial x_{3}}\right|^{-2}$, which yields some difficulty in the estimation of the principal terms.

Third, for the equation of state, there have been a number of arguments in oceanography. A polynomial fit was first proposed by Bryan and Cox [10], which is still one of the effective models. In that work, they proposed that the density is represented as a polynomial of temperature, salinity and height at each point. In UNESCO [52], they determined a model for the equation of state for the ocean, in which the density is provided as a function of the temperature, salinity and pressure. Therefore, we adopt in the present paper a general form of the equation of state $\varrho=\varrho(p, T, S)$, while all of the existing results in mathematical arguments have adopted the polynomial model for the equation of state (see, for instance, [34]). The fourth feature of our model is that it describes the balances of the heat flux at the ocean surface and bottom as those for the potential temperature. Since the equation is described for the in situ temperature, we have to translate the boundary conditions into those for the in situ temperature. As in the preceding works, we construct a strong local-in-time solution in the anisotropic Sobolev-Slobodetskiu spaces.

This paper is organized as follows: in the first section, we formulate the problem. In Section 2, we define the function spaces used throughout this paper. The main result of this paper is stated in Section 3. Auxiliary lemmas, which are important in proving the main theorem, are prepared in Section 4. Section 5 concerns the linear problem, followed by the proof of the main theorem, provided in Section 6 .

\section{Formulation OF THE PROBLEM}

\subsection{Mathematical formulation}

There exist numerous formulations of the vertical coordinate systems of the ocean model, such as those with $z_{-}, \sigma_{-}$, and $\varrho$-coordinates. Although there exist some contributions applicable to the free ocean surface model in $\sigma$-coordinates like COCO [22], Griffies [16] pointed out that the $z$-coordinate system is well suited to representing the topography of the ocean bottom, as well as the movement of the free ocean surface.

Nowadays, the $z$-coordinate system is employed with numerous free ocean surface models, such as the ocean circulation and climate advanced modelling (OCCAM) project, the océan parallélisé (OPA) model, and so on.

Therefore, our problem is formulated in the three-dimensional strip-like region in the $z$-coordinate system mainly based on the formulations in MOM3 [40] and MOM4 [41].

For the temperature in the model equation, however, we adopt the in situ temperature, since the parameterization of the diffusivity coefficient is investigated using it, rather than the potential temperature $[29,31,42]$. On the other hand, the boundary conditions are formulated by using the potential temperature, as we will show later.

Hereafter, by $x=\left(x_{1}, x_{2}, x_{3}\right)=\left(x^{\prime}, x_{3}\right)$, we denote an orthogonal Cartesian coordinate system with $x_{3}$ being the vertical direction. Let the unknown free surface and the known bottom of the ocean be represented by the equations $x_{3}=F\left(x^{\prime}, t\right)$ and $x_{3}=b\left(x^{\prime}\right)$, respectively. The initial value $F_{0}\left(x^{\prime}\right)$ of $F\left(x^{\prime}, t\right)$ is assumed to satisfy $F_{0}\left(x^{\prime}\right)-b\left(x^{\prime}\right)>c_{0}$ with a positive constant $c_{0}$ for any $x^{\prime} \in \mathbf{R}^{2}$. Then the domain $\Omega(t)$ of the ocean at time $t$ is represented as $\left\{\left(x^{\prime}, x_{3}\right) \mid x^{\prime} \in \mathbf{R}^{2}, b\left(x^{\prime}\right)<x_{3}<F\left(x^{\prime}, t\right)\right\}$. Making use of Boussinesq and hydrostatic 
approximations,

$$
\left\{\begin{array}{l}
\frac{\partial \mathbf{v}}{\partial t}+(\mathbf{v} \cdot \nabla) \mathbf{v}+w \frac{\partial \mathbf{v}}{\partial x_{3}}-\left[\mu_{1} \Delta \mathbf{v}+\mu_{2} \frac{\partial^{2} \mathbf{v}}{\partial x_{3}^{2}}\right]+f \mathbf{A v}=-\frac{1}{\varrho_{0}} \nabla p \\
\frac{\partial p}{\partial x_{3}}=-\varrho g \\
\nabla \cdot \mathbf{v}+\frac{\partial w}{\partial x_{3}}=0, \\
\frac{\partial T}{\partial t}+(\mathbf{v} \cdot \nabla) T+w \frac{\partial T}{\partial x_{3}}-\left[\mu_{3} \Delta T+\mu_{4} \frac{\partial^{2} T}{\partial x_{3}^{2}}\right]=0 \\
\frac{\partial S}{\partial t}+(\mathbf{v} \cdot \nabla) S+w \frac{\partial S}{\partial x_{3}}-\left[\mu_{5} \Delta S+\mu_{6} \frac{\partial^{2} S}{\partial x_{3}^{2}}\right]=0 \\
\varrho=\varrho(p, T, S) \quad x \in \Omega(t), t>0 .
\end{array}\right.
$$

Here, $f \mathbf{A v}$ is a Coriolis force with $\mathbf{A}=\left(\begin{array}{cc}0 & -1 \\ 1 & 0\end{array}\right)$ and the Coriolis parameter $f$ is a positive constant due to the $f$-approximation; $\nabla$ and $\Delta$ are two-dimensional gradient and Laplacian, respectively. The horizontal component of the velocity is represented by $\mathbf{v}=\left(v_{1}, v_{2}\right)^{\mathrm{T}}$ and the vertical component $w ; p$ is the pressure; $\varrho=\varrho\left(z_{1}, z_{2}, z_{3}\right)$ is the density; $\varrho_{0}$ is a positive constant; $g$ is the gravity force (a positive constant); $T$ is the temperature; $S$ is the salinity; $\mu_{1}$ and $\mu_{2}$ are the coefficients of turbulent viscosity; and $\left(\mu_{3}, \mu_{4}\right)$ and $\left(\mu_{5}, \mu_{6}\right)$ are given by scaling the sums of the turbulent and molecular diffusivity, respectively. Note that the equation of state is provided in a general form in $(1.1)_{6}$ (hereafter, we represent the $i$ th equation of (a.b) by $(a . b)_{i}$ ). The basic form of this formulation was first derived by Bryan [9], and the discretized code has been widely used in numerical calculations.

On the other hand, numerous arguments carried on him largely from two viewpoints: one is the formulation of the ocean surface, and the other, the parameterization of turbulent viscosity and diffusivity coefficients.

As for ocean surface modeling, Bryan [9] adopted the so-called rigid lid hypothesis, which assumes that the ocean surface is flat and fixed. This makes it easy to separate the behavior of the barotropic and baroclinic flows in the numerical calculation as well as removing the effect of the gravitational wave. However, it imposes the assumption $w=0$ on the ocean surface and the vanishing of fresh water flux, which is a strict restriction.

Later, Crowley [13,14], Blumberg and Mellor [7], Dukowicz and Smith [15] and Killworth [30] developed the free surface model for the ocean. As stated in MOM3 [40], the main advantage of applying the free surface model is the presence of the fresh water flux in the model [16]. In it, the kinematic condition is described as follows [18]:

$$
\frac{\mathrm{D}}{\mathrm{D} t}\left(x_{3}-F\left(x^{\prime}, t\right)\right)=-q_{w}\left(x^{\prime}, F\left(x^{\prime}, t\right), t\right),
$$

where $\frac{\mathrm{D}}{\mathrm{D} t} \equiv \frac{\partial}{\partial t}+\mathbf{v} \cdot \nabla+w \frac{\partial}{\partial x_{3}}$ is an operator known as the material derivative, and $q_{w}$, a function defined on $\mathbf{R}^{3} \times(0, \infty)$, which represents the flux of the fresh water passing across the free ocean surface. It is also represented as

$$
q_{w}=P-E+R,
$$

where $P, E$ and $R$ are the flux of the fresh water entering and leaving the ocean, and river run off, respectively [18]. The process on the ocean surface $\Gamma(t) \equiv\left\{\left(x^{\prime}, F\left(x^{\prime}, t\right)\right) \mid x^{\prime} \in \mathbf{R}^{2}\right\}$ is represented as the balance of the fluxes. The momentum stress on the ocean surface arises from the wind stress and the fresh water momentum in the ocean, and the balance is represented as follows (see (7.60) in MOM3 [40]):

$$
\mu_{1} \nabla F \cdot \nabla \mathbf{v}-\mu_{2} \frac{\partial \mathbf{v}}{\partial x_{3}}-q_{w} \mathbf{v}=\tau_{1},
$$


where $\tau_{1} \in \mathbf{R}^{2}$ is the vertical turbulent momentum flux in the atmosphere-ocean boundary layer, which consists of turbulent stress from winds and the momentum due to fresh water entrained in the winds. $\nabla F \cdot \nabla \mathbf{v}$ in $(1.3)$ stands for a vector whose $i$ th component is $\nabla F \cdot \nabla v_{i}(i=1,2)$. The boundary conditions of the temperature and salinity are described as the conservation of the tracer flux. Representing the tracer concentration of a substance by $U$, the total concentration flux across the ocean surface is the sum of the change in the tracer concentration due to the behavior of the fresh water and the diffusivity [17]:

$$
U q_{w}+\mathbf{F}_{U} \cdot \mathbf{N}_{F},
$$

where $\mathbf{F}_{U}$ is the diffusive tracer flux, and $\mathbf{N}_{F}=(-\nabla F, 1)^{\mathrm{T}} \in \mathbf{R}^{3}$ is the upward normal vector to the free ocean surface.

It is well known that, as for the temperature, the potential temperature rather than the in situ temperature satisfies $(1.4)[18,40]$. In general, the potential temperature $\theta$ depends on the pressure, in situ temperature and salinity:

$$
\theta=\theta(p, T, S) .
$$

Griffies [18] also pointed out that, as for the potential temperature, the first term of (1.4) does not differ so much with respect to depth, which he calls the neutral tracer. From these considerations, the boundary conditions for the temperature and salinity are described as follows [3,18]:

$$
\mathbf{F}_{T} \cdot \mathbf{N}_{F}=\tau_{2}, \quad \mathbf{F}_{S} \cdot \mathbf{N}_{F}-q_{w} S=0, \quad p=p_{0} \quad x \in \Gamma(t), t>0,
$$

where

$$
\mathbf{F}_{T}=\mathbf{K}_{2} \nabla_{3} \theta, \quad \mathbf{F}_{S}=\mathbf{K}_{3} \nabla_{3} S,
$$

with the three-dimensional gradient operator $\nabla_{3}$ and

$$
\mathbf{K}_{i}=\left[\begin{array}{lll}
\mu_{2 i-1} & 0 & 0 \\
0 & \mu_{2 i-1} & 0 \\
0 & 0 & \mu_{2 i}
\end{array}\right] \quad(i=1,2,3) .
$$

In $(1.5)_{1}, \tau_{2}$ is the outward heat fluxes on the ocean surface, and $p_{0}\left(x^{\prime}, t\right)$, the pressure on the ocean surface, formalized as a function defined on the two-dimensional Euclid region and time.

Remark 1.1. In Bryan's model, although it applies the rigid-lid hypothesis, Dirichlet boundary conditions are also admitted for temperature and salinity (see [9], p. 156) in place of the Neumann type condition on the ocean surface:

$$
(T, S)=\left(T_{e}, S_{e}\right) \quad x \in \Gamma(t) .
$$

In the present paper, we concentrate on Neumann-type conditions, (1.3) and (1.5), on the ocean surface. Actually, under appropriate assumptions, it is possible to obtain a local-in-time solution for both types of conditions. This holds for the linear problem that we will discuss in Section 5.

At the bottom of the ocean, stress arises from those conditions associated with the bottom topography and sub-grid scale effects [40]. In MOM3, the latter is ignored in its default configuration, and we adopt here the formulation (see (7.51) in MOM3)

$$
\mu_{1} \nabla b \cdot \nabla \mathbf{v}+\mu_{2} \frac{\partial \mathbf{v}}{\partial x_{3}}=\tau_{3}
$$

Here $\tau_{3}$ is the bottom stress, and the term $\nabla b \cdot \nabla \mathbf{v}$ is interpreted as the term $\nabla F \cdot \nabla \mathbf{v}$ in (1.3) is.

It is also necessary to impose the bottom kinematic boundary condition (see MOM3 [40] (4.24), (7.15)):

$$
w=-\mathbf{v} \cdot \nabla b .
$$


As for the temperature and salinity, the tracer flux is set to zero (see Sect. 4.3.4 in MOM3 [40]):

$$
\mathbf{F}_{T} \cdot \mathbf{N}_{b}=0, \quad \mathbf{F}_{S} \cdot \mathbf{N}_{b}=0 \quad x \in \Gamma_{b}, t>0,
$$

where $\mathbf{N}_{b}=(-\nabla b, 1)^{\mathrm{T}}$.

Next, let us discuss the parameterization of the turbulent viscosity and diffusivity coefficients in the vertical direction, $\mu_{i}(i=2,4,6)$. Based on measurements, it has been pointed out that these depend on the Richardson number $[29,31]$, especially where the vertical mixing of seawater is vigorous. In the present paper, following the parameterization by Pacanowski and Philander $[42,53]$, the vertical turbulent viscosity and diffusivity coefficients are represented as follows:

$$
\left\{\begin{array}{l}
\mu_{2}=\mu_{2}\left(\frac{\partial \mathbf{v}}{\partial x_{3}}, \varrho, \frac{\partial \varrho}{\partial x_{3}}\right)=\mu_{2 a}\left(1+\alpha_{2} \mathcal{R}\left(\frac{\partial \mathbf{v}}{\partial x_{3}}, \varrho, \frac{\partial \varrho}{\partial x_{3}}\right)\right)^{-2}+\mu_{2 b}, \\
\mu_{i}=\mu_{i}\left(\frac{\partial \mathbf{v}}{\partial x_{3}}, \varrho, \frac{\partial \varrho}{\partial x_{3}}\right)=\mu_{i a}\left(1+\alpha_{i} \mathcal{R}\left(\frac{\partial \mathbf{v}}{\partial x_{3}}, \varrho, \frac{\partial \varrho}{\partial x_{3}}\right)\right)^{-1}+\mu_{i b} \quad(i=4,6), \\
\mathcal{R}=\mathcal{R}\left(\frac{\partial \mathbf{v}}{\partial x_{3}}, \varrho, \frac{\partial \varrho}{\partial x_{3}}\right)=g \varrho^{-1} \frac{\partial \varrho}{\partial x_{3}}\left|\frac{\partial \mathbf{v}}{\partial x_{3}}\right|^{-2},
\end{array}\right.
$$

where $\mu_{i a}, \mu_{i b}(i=2,4,6)$ are positive constants, and $\mathcal{R}$ is the Richardson number. On the other hand, $\mu_{i}(i=1,3,5)$ are formulated as positive constants. It is worth noting that formulations of $\mu_{4}$ and $\mu_{6}$ are provided as the diffusivity of the in situ temperature, and therefore we have to rewrite the boundary conditions $(1.5)_{1}$ and $(1.8)_{1}$ as those for the in situ temperature.

Finally, the initial conditions are provided by

$$
(\mathbf{v}, T, S)(x, 0)=\left(\mathbf{v}_{0}, T_{0}, S_{0}\right)(x) \quad x \in \Omega \equiv \Omega(0), \quad F\left(x^{\prime}, 0\right)=F_{0}\left(x^{\prime}\right) \quad x^{\prime} \in \mathbf{R}^{2} .
$$

In order to consider the differentiation of the current and initial value of the horizontal velocity, we first extend $\mathbf{v}_{0}=\left(v_{01}, v_{02}\right)^{\mathrm{T}}$ into the whole space $\mathbf{R}^{3}$ preserving the regularity $[32,54]$, which is denoted by $\overline{\mathbf{v}}_{0}$, and then introduce the notation $\mathbf{v}^{\prime}=\mathbf{v}-\overline{\mathbf{v}}_{0}$. Hereafter we also use a notation $\mathcal{V}_{x} \equiv(p, T, S)^{\mathrm{T}}$, for simplicity.

Then, the original problem of (1.1)-(1.9) becomes as follows:

$$
\left\{\begin{array}{l}
\frac{\partial \mathbf{v}^{\prime}}{\partial t}+\left(\left(\mathbf{v}^{\prime}+\overline{\mathbf{v}}_{0}\right) \cdot \nabla\right) \mathbf{v}^{\prime}+w \frac{\partial \mathbf{v}^{\prime}}{\partial x_{3}}-\left(\mu_{1} \triangle \mathbf{v}^{\prime}+\mu_{2} \frac{\partial^{2} \mathbf{v}^{\prime}}{\partial x_{3}^{2}}\right) \\
=-f \mathbf{A}\left(\mathbf{v}^{\prime}+\overline{\mathbf{v}}_{0}\right)-\frac{1}{\varrho_{0}} \nabla p-\left(\left(\mathbf{v}^{\prime}+\overline{\mathbf{v}}_{0}\right) \cdot \nabla\right) \overline{\mathbf{v}}_{0}-w \frac{\partial \overline{\mathbf{v}}_{0}}{\partial x_{3}}+\left(\mu_{1} \triangle \overline{\mathbf{v}}_{0}+\mu_{2} \frac{\partial^{2} \overline{\mathbf{v}}_{0}}{\partial x_{3}^{2}}\right) \\
\frac{\partial T}{\partial t}+\left(\left(\mathbf{v}^{\prime}+\overline{\mathbf{v}}_{0}\right) \cdot \nabla\right) T+w \frac{\partial T}{\partial x_{3}}-\left[\mu_{3} \Delta T+\mu_{4} \frac{\partial^{2} T}{\partial x_{3}^{2}}\right]=0, \\
\frac{\partial S}{\partial t}+\left(\left(\mathbf{v}^{\prime}+\overline{\mathbf{v}}_{0}\right) \cdot \nabla\right) S+w \frac{\partial S}{\partial x_{3}}-\left[\mu_{5} \Delta S+\mu_{6} \frac{\partial^{2} S}{\partial x_{3}^{2}}\right]=0 \\
\frac{\partial w}{\partial x_{3}}=-\nabla \cdot\left(\mathbf{v}^{\prime}+\overline{\mathbf{v}}_{0}\right), \\
\frac{\partial p}{\partial x_{3}}=-g \varrho(p, T, S) \quad x \in \Omega(t), t>0
\end{array}\right.
$$




$$
\begin{aligned}
& \frac{\mathrm{D}}{\mathrm{D} t}\left(x_{3}-F\left(x^{\prime}, t\right)\right)=-q_{w}\left(x^{\prime}, F\left(x^{\prime}, t\right), t\right) \quad x^{\prime} \in \mathbf{R}^{2}, t>0, \\
& \left\{\begin{array}{l}
\mu_{1} \nabla \mathbf{v}^{\prime} \cdot \nabla F-\mu_{2} \frac{\partial \mathbf{v}^{\prime}}{\partial x_{3}}-q_{w} \mathbf{v}^{\prime}=\tau_{1}-\mu_{1} \nabla \overline{\mathbf{v}}_{0} \cdot \nabla F+\mu_{2} \frac{\partial \overline{\mathbf{v}}_{0}}{\partial x_{3}}+q_{w} \overline{\mathbf{v}}_{0}, \\
\mu_{3} \sum_{i=1}^{2} \nabla_{3} \theta\left(\mathcal{V}_{x}\right) \cdot \frac{\partial \mathcal{V}_{x}}{\partial x_{i}} \frac{\partial F}{\partial x_{i}}-\mu_{4} \nabla_{3} \theta\left(\mathcal{V}_{x}\right) \cdot \frac{\partial \mathcal{V}_{x}}{\partial x_{3}}=\tau_{2}, \\
\mu_{5} \nabla S \cdot \nabla F-\mu_{6} \frac{\partial S}{\partial x_{3}}-q_{w} S=0, \quad p=p_{0} \quad x \in \Gamma(t), t>0,
\end{array}\right. \\
& \left\{\begin{array}{l}
\mu_{1} \nabla \mathbf{v}^{\prime} \cdot \nabla b-\mu_{2} \frac{\partial \mathbf{v}^{\prime}}{\partial x_{3}}=\tau_{3}-\mu_{1} \nabla \overline{\mathbf{v}}_{0} \cdot \nabla b+\mu_{2} \frac{\partial \overline{\mathbf{v}}_{0}}{\partial x_{3}}, \\
w=-\left(\mathbf{v}^{\prime}+\mathbf{v}_{0}\right) \cdot \nabla b, \\
\mu_{3} \sum_{i=1}^{2} \nabla_{3} \theta\left(\mathcal{V}_{x}\right) \cdot \frac{\partial \mathcal{V}_{x}}{\partial x_{i}} \frac{\partial b}{\partial x_{i}}-\mu_{4} \nabla_{3} \theta\left(\mathcal{V}_{x}\right) \cdot \frac{\partial \mathcal{V}_{x}}{\partial x_{3}}=0, \\
\mu_{5} \nabla S \cdot \nabla b-\mu_{6} \frac{\partial S}{\partial x_{3}}=0 \quad x \in \Gamma_{b}, t>0,
\end{array}\right. \\
& \left(\mathbf{v}^{\prime}, T, S\right)(x, 0)=\left(\mathbf{0}, T_{0}, S_{0}\right)(x) \quad x \in \Omega \equiv \Omega(0), \quad F\left(x^{\prime}, 0\right)=F_{0}\left(x^{\prime}\right) \quad x^{\prime} \in \mathbf{R}^{2} .
\end{aligned}
$$

Hereafter, we will consider the problem of (1.10)-(1.14). Before solving it, we introduce a coordinate transform to make the region into the fixed one in the next subsection.

\subsection{Coordinate transform}

The problem stated in the previous subsection is considered by applying the transform $\Phi_{F}:(x, t) \longmapsto\left(y, t^{*}\right)$ of a coordinate system similar to the one used in preceding papers $[25,26]$ to $(1.10)-(1.14)$. This enables us to consider the problem in a fixed region.

$$
y^{\prime}=x^{\prime}, \quad y_{3}=\left(b\left(x^{\prime}\right)-F_{0}\left(x^{\prime}\right)\right) \frac{x_{3}-F\left(x^{\prime}, t\right)}{b\left(x^{\prime}\right)-F\left(x^{\prime}, t\right)}+F_{0}\left(x^{\prime}\right), \quad t^{*}=t .
$$

This transform is also similar to those used in $[2,47]$. It is clear that for arbitrary $T_{1}>0$, the regions

$$
\bigcup_{0<t<T_{1}}(\Omega(t) \times\{t\}), \bigcup_{0<t<T_{1}}\left(\Gamma_{b} \times\{t\}\right), \bigcup_{0<t<T_{1}}(\Gamma(t) \times\{t\})
$$

are transformed onto the regions $\Omega_{T_{1}} \equiv \Omega \times\left(0, T_{1}\right), \Gamma_{b T_{1}} \equiv \Gamma_{b} \times\left(0, T_{1}\right), \Gamma_{T_{1}} \equiv \Gamma(0) \times\left(0, T_{1}\right)$, respectively. In the following, we use the representation

$$
\left(x^{\prime}, x_{3}, t\right)=\left(y^{\prime}, \frac{\left(y_{3}-F_{0}\left(y^{\prime}\right)\right)\left(b\left(y^{\prime}\right)-F\left(y^{\prime}, t\right)\right)}{b\left(y^{\prime}\right)-F_{0}\left(y^{\prime}\right)}+F\left(y^{\prime}, t\right), t\right) \equiv\left(y^{\prime}, X_{3}^{(F)}(y, t), t\right)=\Phi_{F}^{-1}(y, t),
$$

and denote the inverse of the transposed matrix of the Jacobian matrix by

$$
\left(J[(x / y)]^{\mathrm{T}}\right)^{-1}=\left(a^{i j}\right)=\left(a^{i j}(F)\right) \quad(i, j=1,2,3) .
$$


Then one can easily derive

$$
\begin{aligned}
& \mathbf{a}^{3}(F) \equiv\left(a^{13}(F), a^{23}(F)\right)^{\mathrm{T}}= \frac{\left(F_{0}\left(y^{\prime}\right)-F\left(y^{\prime}, t\right)\right)\left(y_{3}-F_{0}\left(y^{\prime}\right)\right)}{\left(b\left(y^{\prime}\right)-F\left(y^{\prime}, t\right)\right)\left(b\left(y^{\prime}\right)-F_{0}\left(y^{\prime}\right)\right)} \nabla b\left(y^{\prime}\right) \\
&+\left(b\left(y^{\prime}\right)-y_{3}\right)\left\{\frac{\nabla F_{0}\left(y^{\prime}\right)}{b\left(y^{\prime}\right)-F_{0}\left(y^{\prime}\right)}-\frac{\nabla F\left(y^{\prime}, t\right)}{b\left(y^{\prime}\right)-F\left(y^{\prime}, t\right)}\right\}, \\
& A_{1}(y, t) \equiv \frac{\partial y}{\partial t}=\frac{y_{3}-b\left(y^{\prime}\right)}{b\left(y^{\prime}\right)-F\left(y^{\prime}, t\right)} \frac{\partial F}{\partial t}\left(y^{\prime}, t\right), \quad a^{33}(F)=\frac{b\left(y^{\prime}\right)-F_{0}\left(y^{\prime}\right)}{b\left(y^{\prime}\right)-F\left(y^{\prime}, t\right)}, \quad a^{i j}=\delta_{i j} \quad(i=1,2,3, j=1,2) .
\end{aligned}
$$

In the following, we use the notation

$$
\left(\begin{array}{c}
\nabla_{F} \\
\nabla_{F, 3}
\end{array}\right)=\left(J[(x / y)]^{\mathrm{T}}\right)^{-1}\left(\begin{array}{c}
\nabla_{y^{\prime}} \\
\frac{\partial}{\partial y_{3}}
\end{array}\right)
$$

where $\nabla_{y^{\prime}}$ is the derivative with respect to $y^{\prime}$. We also use the notation

$$
\tilde{f}^{(F)}(y, t) \equiv f\left(\Phi_{F}^{-1}(y, t)\right)=f\left(y^{\prime}, X_{3}^{(F)}(y, t), t\right)
$$

in order to represent the dependency of the coordinate transform on $F$. For a function defined in the whole space $\mathbf{R}^{3}$, we use the same notation to the one restricted on $\Omega(t)$ at each $t$ and then transformed into the new coordinate system.

\section{FUnCTION SPACES}

Before proceeding to the main theorem, let us introduce some function spaces used in this paper. Let $G$ be a simply connected domain in $\mathbf{R}^{n}(n=2,3)$ in general. In the following, we mean the derivative of function $f$ with multi-index $\alpha$ by $D^{\alpha} f$. The $\chi_{1}$ th order derivative with respect to $t$ and $\alpha$ with respect to $y$ is denoted by $D_{t}^{\chi_{1}} D_{y}^{\alpha} f$. For notations of function spaces, we basically follow those used in preceding contributions from Solonnikov [45], Tani [46], and Wloka [54]. By $W_{2}^{l}(G)$, we mean a space of functions $u(x), x \in G$ equipped with the norm $\|u\|_{W_{2}^{l}(G)}^{2}=\sum_{|\alpha|<l}\left\|D^{\alpha} u\right\|_{L_{2}(G)}^{2}+\|u\|_{\dot{W}_{2}^{l}(G)}^{2}$, where

$$
\left\{\begin{array}{l}
\|u\|_{\dot{W}_{2}^{l}(G)}^{2}=\sum_{|\alpha|=l}\left\|D^{\alpha} u\right\|_{L_{2}(G)}^{2}=\sum_{|\alpha|=l} \int_{G}\left|D^{\alpha} u(x)\right|^{2} \mathrm{~d} x \quad \text { if } l \text { is an integer, } \\
\|u\|_{\dot{W}_{2}^{l}(G)}^{2}=\sum_{|\alpha|=[l]} \int_{G} \int_{G} \frac{\left|D^{\alpha} u(x)-D^{\alpha} u(y)\right|^{2}}{|x-y|^{n+2\{l\}}} \mathrm{d} x \mathrm{~d} y \quad \text { if } l \text { is a non-integer, } l=[l]+\{l\}, 0<\{l\}<1 .
\end{array}\right.
$$

We also define the following function spaces for $m>1$ :

$$
\bar{W}_{2}^{m}(G)=\left\{u(x)=\left.o\left(\mathrm{e}^{x^{2}}\right)\left|\|u\|_{\bar{W}_{2}^{m}(G)}^{2} \equiv \sup _{x \in G}\right| u(x)\right|^{2}+\|u\|_{\dot{W}_{2}^{m-[m]}(G)}^{2}+\sum_{|\alpha|=1}\left\|D^{\alpha} u\right\|_{W_{2}^{m-1}(G)}^{2}<\infty\right\},
$$


and for $m>2$,

$$
\begin{aligned}
\bar{W}_{2, c}^{m}(G)= & \left\{u(x)=\left.o\left(\mathrm{e}^{x^{2}}\right)\left|\|u\|_{\bar{W}_{2}^{m}(G)}^{2} \equiv \sup _{x \in G,|\alpha| \leq 1}\right| D^{\alpha} u(x)\right|^{2}+\sum_{|\alpha| \leq 1}\left\|D^{\alpha} u\right\|_{\dot{W}_{2}^{m-[m]}(G)}^{2}\right. \\
& \left.+\sum_{|\alpha|=2}\left\|D^{\alpha} u\right\|_{W_{2}^{m-2}(G)}^{2}<\infty\right\} .
\end{aligned}
$$

Next, we introduce anisotropic Sobolev-Slobodetskiı̌ spaces [54] $W_{2}^{l, \frac{l}{2}}\left(G_{T_{1}}\right) \equiv W_{2}^{l, 0}\left(G_{T_{1}}\right) \cap W_{2}^{0, \frac{l}{2}}\left(G_{T_{1}}\right)\left(G_{T_{1}} \equiv\right.$ $\left.G \times\left(0, T_{1}\right)\right)$, whose norms are defined by

$$
\|u\|_{W_{2}^{l, \frac{l}{2}}\left(G_{T_{1}}\right)}^{2}=\int_{0}^{T_{1}}\|u(\cdot, t)\|_{W_{2}^{l}(G)}^{2} \mathrm{~d} t+\int_{G}\|u(x, \cdot)\|_{W_{2}^{\frac{l}{2}\left(0, T_{1}\right)}}^{2} \mathrm{~d} x \equiv\|u\|_{W_{2}^{l, 0}\left(G_{T_{1}}\right)}^{2}+\|u\|_{W_{2}^{0, \frac{l}{2}}\left(G_{T_{1}}\right)}^{2} .
$$

We also define function spaces for $m>2$,

$$
\begin{aligned}
& \bar{W}_{2}^{m, \frac{m}{2}}\left(G_{T_{1}}\right)=\left\{u(x, t)=\left.o\left(\mathrm{e}^{x^{2}}\right) \forall t \in\left(0, T_{1}\right)\left|\|u\|_{\bar{W}_{2}^{m, \frac{m}{2}}\left(G_{T_{1}}\right)}^{2} \equiv \sup _{G_{T_{1}}}\right| u(x, t)\right|^{2}\right.
\end{aligned}
$$

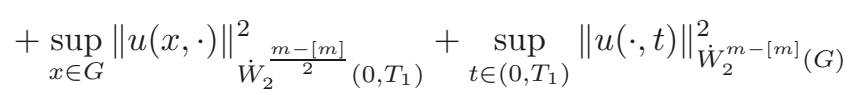

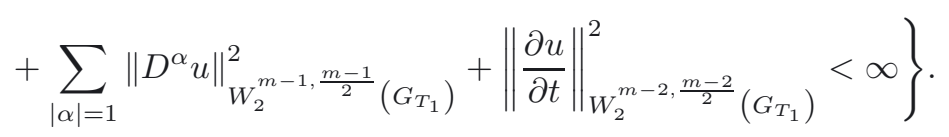

$$
\begin{aligned}
& \bar{W}_{2, c}^{m, \frac{m}{2}}\left(G_{T_{1}}\right)=\left\{u(x, t)=\left.o\left(\mathrm{e}^{x^{2}}\right) \forall t \in\left(0, T_{1}\right)\left|\|u\|_{\bar{W}_{2, c}^{m, \frac{m}{2}}\left(G_{T_{1}}\right)}^{2} \equiv \sup _{\substack{G_{T_{1}} \\
|\alpha| \leq 1}}\right| D^{\alpha} u(x, t)\right|^{2}\right.
\end{aligned}
$$

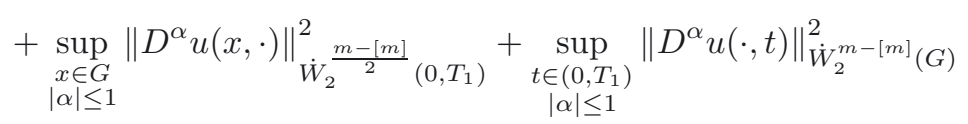

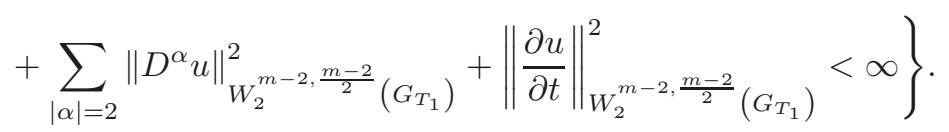

The $n$ times product of a function space $W_{0}$ is denoted by $W_{0}^{n}$, and we also use notations like $\prod_{i=1}^{2} W_{i} \equiv W_{1} \times W_{2}$ hereafter. Norms of the vector and the product spaces are defined by the standard vector norm and the sum of the norms of each space, respectively.

\section{MAin RESUlT}

In this section, we first rewrite the problem shown in Section 1 in the new coordinate system, and then state the local-in-time solvability of it, which is the main result of the present paper. Since we define numerous functions spaces throughout this paper, we list them in the appendix.

\subsection{Problem in the new coordinate system}

We introduce the problem after the coordinate transform. By noting that the coordinate system does not vary at $t=0$, we rewrite the problem from (1.10)-(1.14) in $y$-coordinates and denote $\left(\mathbf{v}^{\prime}, w, T, S, \mathcal{V}_{x}\right)$ after the 
coordinate transform with $\left(\mathbf{u}^{\prime}, u_{3}, \tilde{T}, \tilde{S}, \mathcal{V}\right)$. We also use notations $\mathbf{u}=\mathbf{u}^{\prime}+\tilde{\mathbf{v}}_{0}^{(F)}, \mathbf{u}=\left(u_{1}, u_{2}\right)^{\mathrm{T}}, \mathcal{U} \equiv\left(\mathbf{u}^{\prime}, \tilde{T}, \tilde{S}\right)^{\mathrm{T}}$, $\mathcal{M} \equiv(\tilde{T}, \tilde{S})^{\mathrm{T}}, \mathcal{U}_{0} \equiv\left(\mathbf{0}, T_{0}, S_{0}\right)^{\mathrm{T}}$ and $\mathbf{P}=\nabla_{F} \tilde{p}^{(F)}$ hereafter. Note that, in the following, we consider that the unknown variables are $\mathbf{u}^{\prime}, \tilde{T}, \tilde{S}, \mathbf{P}$ and $F$, since $u_{3}$ is represented by using $\mathbf{u}^{\prime}$ and $F$, as we will see later:

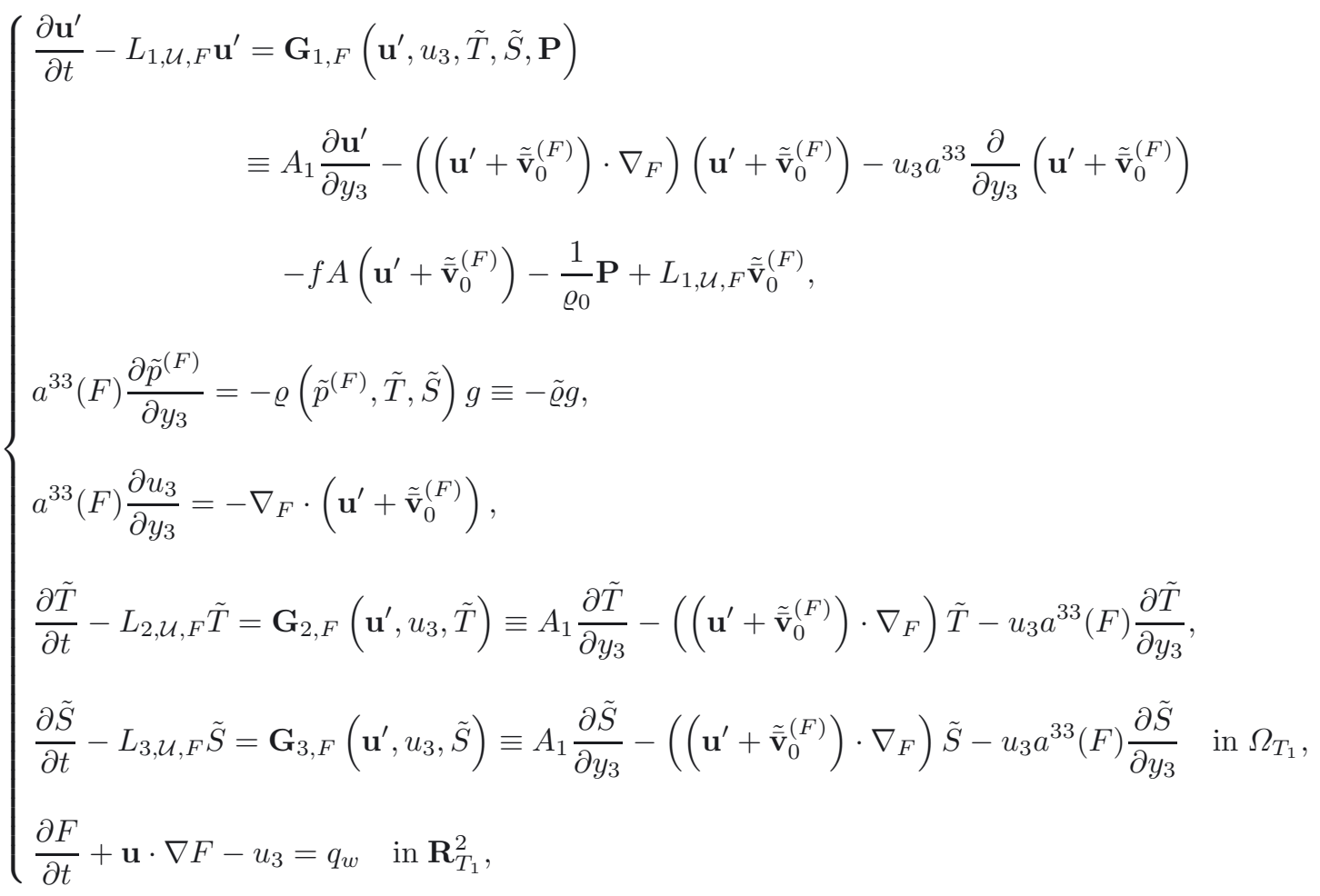

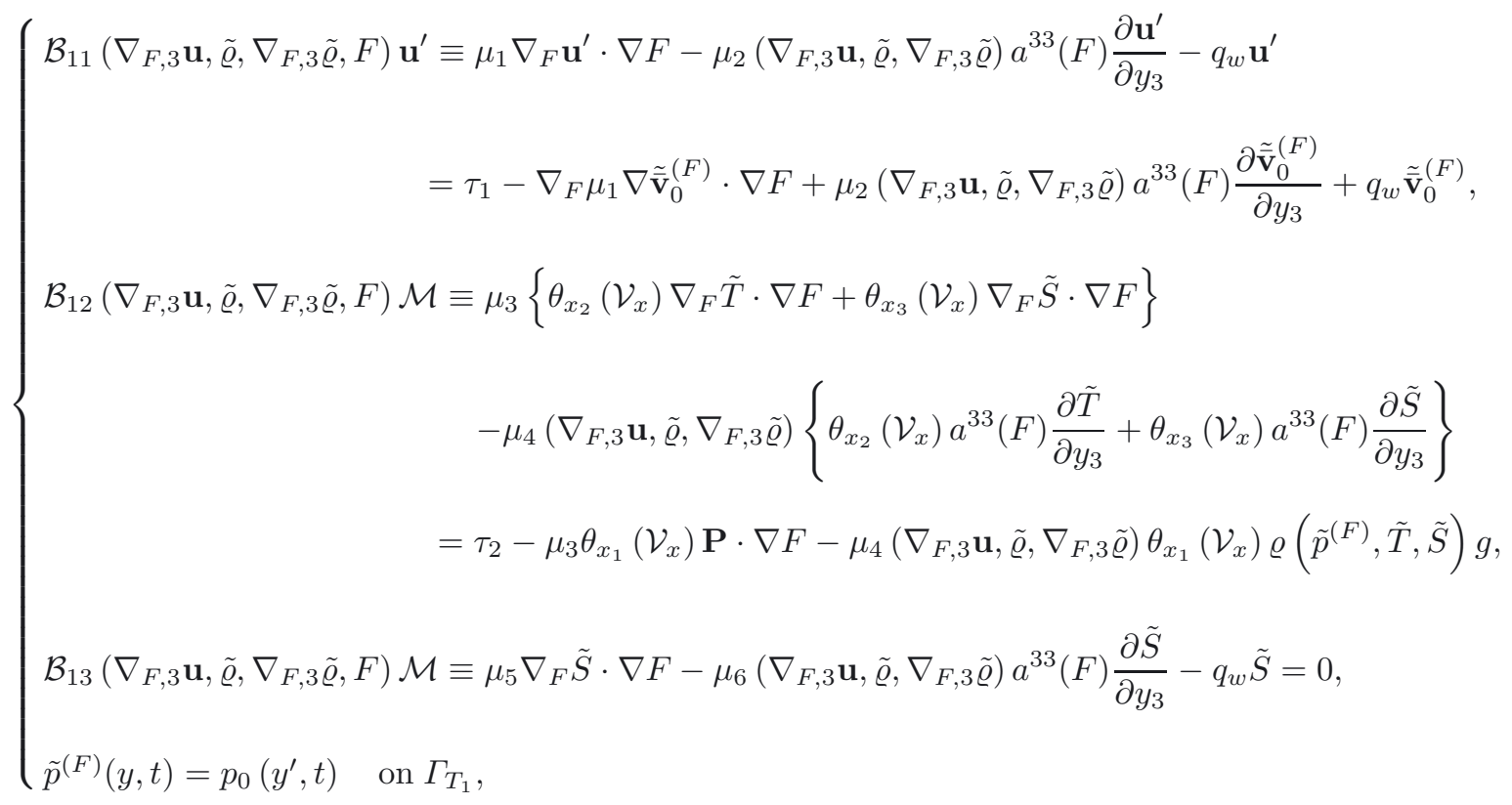




$$
\begin{aligned}
& \left(\mathcal{B}_{21}\left(\nabla_{F, 3} \mathbf{u}, \tilde{\varrho}, \nabla_{F, 3} \tilde{\varrho}, F\right) \mathbf{u}^{\prime} \equiv \mu_{1} \nabla_{F} \mathbf{u}^{\prime} \cdot \nabla b-\mu_{2}\left(\nabla_{F, 3} \mathbf{u}, \tilde{\varrho}, \nabla_{F, 3} \tilde{\varrho}\right) a^{33}(F) \frac{\partial \mathbf{u}^{\prime}}{\partial y_{3}}\right. \\
& =\tau_{3}-\mu_{1} \nabla_{F} \tilde{\overline{\mathbf{v}}}_{0}^{(F)} \cdot \nabla b+\mu_{2}\left(\nabla_{F, 3} \mathbf{u}, \tilde{\varrho}, \nabla_{F, 3} \tilde{\varrho}\right) a^{33}(F) \frac{\partial \tilde{\mathbf{v}}_{0}^{(F)}}{\partial y_{3}}, \\
& \mathcal{B}_{22}\left(\nabla_{F, 3} \mathbf{u}, \tilde{\varrho}, \nabla_{F, 3} \tilde{\varrho}, F\right) \mathcal{M} \equiv \mu_{3}\left\{\theta_{x_{2}}\left(\mathcal{V}_{x}\right) \nabla_{F} \tilde{T} \cdot \nabla b+\theta_{x_{3}}\left(\mathcal{V}_{x}\right) \nabla_{F} \tilde{S} \cdot \nabla b\right\} \\
& -\mu_{4}\left(\nabla_{F, 3} \mathbf{u}, \tilde{\varrho}, \nabla_{F, 3} \tilde{\varrho}\right)\left\{\theta_{x_{2}}\left(\mathcal{V}_{x}\right) a^{33}(F) \frac{\partial \tilde{T}}{\partial y_{3}}+\theta_{x_{3}}\left(\mathcal{V}_{x}\right) a^{33}(F) \frac{\partial \tilde{S}}{\partial y_{3}}\right\} \\
& =-\mu_{3} \theta_{x_{1}}\left(\mathcal{V}_{x}\right) \mathbf{P} \cdot \nabla b-\mu_{4}\left(\nabla_{F, 3} \mathbf{u}, \tilde{\varrho}, \nabla_{F, 3} \tilde{\varrho}\right) \theta_{x_{1}}\left(\mathcal{V}_{x}\right) \varrho\left(\tilde{p}^{(F)}, \tilde{T}, \tilde{S}\right) g \\
& u_{3}=-\left(\mathbf{u}^{\prime}+\mathbf{v}_{0}\right) \cdot \nabla b \\
& \mathcal{B}_{23}\left(\nabla_{F, 3} \mathbf{u}, \tilde{\varrho}, \nabla_{F, 3} \tilde{\varrho}, F\right) \mathcal{M} \equiv \mu_{5} \nabla_{F} \tilde{S} \cdot \nabla b-\mu_{6}\left(\nabla_{F, 3} \mathbf{u}, \tilde{\varrho}, \nabla_{F, 3} \tilde{\varrho}\right) a^{33}(F) \frac{\partial \tilde{S}}{\partial y_{3}}=0 \quad \text { on } \Gamma_{b T_{1}}, \\
& \left\{\begin{array}{l}
\left.\mathcal{U}\right|_{t=0}(y)=\mathcal{U}_{0}(y) \equiv\left(\mathbf{0}, T_{0}(y), S_{0}(y)\right)^{\mathrm{T}} \quad \text { on } \Omega \\
\left.F\right|_{t=0}=F_{0}\left(y^{\prime}\right) \quad \text { on } \mathbf{R}^{2}
\end{array}\right.
\end{aligned}
$$

where

$$
L_{i, \mathcal{U}, F} \equiv \mu_{2 i-1} \nabla_{F}^{2}+\mu_{2 i}\left(\nabla_{F, 3} \mathbf{u}, \tilde{\varrho}, \nabla_{F, 3} \tilde{\varrho}\right)\left(a^{33}(F)\right)^{2} \frac{\partial^{2}}{\partial y_{3}^{2}} \quad(i=1,2,3) .
$$

Note that $u_{3}$ is deduced from $(3.1)_{3}$ and $(3.3)_{3}$ :

$$
\left\{\begin{array}{l}
a^{33}(F) \frac{\partial u_{3}}{\partial y_{3}}=-\nabla_{F} \cdot\left(\mathbf{u}^{\prime}+\tilde{\mathbf{v}}_{0}^{(F)}\right) \quad \text { in } \Omega_{T}, \\
u_{3}=-\left(\mathbf{u}^{\prime}+\mathbf{v}_{0}\right) \cdot \nabla b \quad \text { on } \Gamma_{b}
\end{array}\right.
$$

whose solution is provided by

$$
u_{3}(y, t)=-\left(\mathbf{u}^{\prime}+\left.\mathbf{v}_{0}\right|_{\Gamma_{b}}\right) \cdot \nabla b\left(y^{\prime}, t\right)-\frac{1}{a^{33}(F)} \int_{b\left(y^{\prime}\right)}^{y_{3}} \nabla_{F} \cdot \mathbf{u}\left(y^{\prime}, z_{3}, t\right) \mathrm{d} z_{3} .
$$

Hereafter, we substitute (3.6) into other equations in (3.1), and replace

$$
\mathbf{G}_{1, F}\left(\mathbf{u}^{\prime}, u_{3}, \tilde{T}, \tilde{S}, \mathbf{P}\right), G_{2, F}\left(\mathbf{u}^{\prime}, u_{3}, \tilde{T}\right), G_{3, F}\left(\mathbf{u}^{\prime}, u_{3}, \tilde{S}\right),
$$

in the right-hand sides by following notations

$$
\mathbf{G}_{1, F}\left(\mathbf{u}^{\prime}, \tilde{T}, \tilde{S}, \mathbf{P}\right), G_{2, F}\left(\mathbf{u}^{\prime}, \tilde{T}\right), G_{3, F}\left(\mathbf{u}^{\prime}, \tilde{S}\right) .
$$

Then, the problem for $\mathcal{U}$ in (3.1)-(3.4) is written as follows:

$$
\begin{cases}\frac{\partial \mathcal{U}}{\partial t}-\mathcal{L}_{\mathcal{U}, F} \mathcal{U}=\mathcal{G}_{1, F, \mathbf{P}} \mathcal{U} \quad \text { in } \Omega_{T_{1}}, & \\ \mathcal{B}_{1}\left(\nabla_{F, 3} \mathbf{u}, \tilde{\varrho}, \nabla_{F, 3} \tilde{\varrho}, F\right) \mathcal{U}=\bar{\tau}_{1}(\mathbf{u}, \mathcal{M}, F, \mathbf{P}) & \text { on } \Gamma_{T_{1}}, \\ \mathcal{B}_{2}\left(\nabla_{F, 3} \mathbf{u}, \tilde{\varrho}, \nabla_{F, 3} \tilde{\varrho}, F\right) \mathcal{U}=\bar{\tau}_{2}(\mathbf{u}, \mathcal{M}, F, \mathbf{P}) & \text { on } \Gamma_{b T_{1}}, \\ \left.\mathcal{U}\right|_{t=0}=\mathcal{U}_{0} \quad \text { on } \Omega, & \end{cases}
$$


where we have introduced notations

$$
\begin{aligned}
& \bar{\tau}_{1}(\mathbf{u}, \mathcal{M}, F, \mathbf{P})=\left(\tau_{1}-\mu_{1} \nabla_{F} \tilde{\overline{\mathbf{v}}}_{0}^{(F)} \cdot \nabla F+\mu_{2}\left(\nabla_{F, 3} \mathbf{u}, \tilde{\varrho}, \nabla_{F, 3} \tilde{\varrho}\right) a^{33}(F) \frac{\partial \tilde{\overline{\mathbf{v}}}_{0}^{(F)}}{\partial y_{3}}+q_{w} \tilde{\overline{\mathbf{v}}}_{0}^{(F)},\right. \\
& \left.\tau_{2}-\mu_{3} \theta_{x_{1}} \nabla_{F} \tilde{p}^{(F)} \cdot \nabla F-\mu_{4}\left(\nabla_{F, 3} \mathbf{u}, \tilde{\varrho}, \nabla_{F, 3} \tilde{\varrho}\right) \theta_{x_{1}} \varrho\left(\tilde{p}^{(F)}, \tilde{T}, \tilde{S}\right) g, 0\right)^{\mathrm{T}}, \\
& \bar{\tau}_{2}(\mathbf{u}, \mathcal{M}, F, \mathbf{P})=\left(\tau_{3}-\mu_{1} \nabla_{F} \tilde{\overline{\mathbf{v}}}_{0}^{(F)} \cdot \nabla b+\mu_{2}\left(\nabla_{F, 3} \mathbf{u}, \tilde{\varrho}, \nabla_{F, 3} \tilde{\varrho}\right) a^{33}(F) \frac{\partial \tilde{\overline{\mathbf{v}}}_{0}^{(F)}}{\partial y_{3}}\right. \\
& \left.-\mu_{3} \theta_{x_{1}} \nabla_{F} \tilde{p}^{(F)} \cdot \nabla b-\mu_{4}\left(\nabla_{F, 3} \mathbf{u}, \tilde{\varrho}, \nabla_{F, 3} \tilde{\varrho}\right) \theta_{x_{1}} \varrho\left(\tilde{p}^{(F)}, \tilde{T}, \tilde{S}\right) g, 0\right)^{\mathrm{T}},
\end{aligned}
$$

and

$$
\mathcal{L}_{\mathcal{U}, F} \mathcal{U} \equiv\left(L_{1, \mathcal{U}, F} \mathbf{u}^{\prime}, L_{2, \mathcal{U}, F} \tilde{T}, L_{3, \mathcal{U}, F} \tilde{S}\right)^{\mathrm{T}}, \quad \mathcal{G}_{1, F, \mathbf{P}} \mathcal{U} \equiv\left(\mathbf{G}_{1, F}\left(\mathbf{u}^{\prime}, \tilde{T}, \tilde{S}, \mathbf{P}\right), G_{2, F}\left(\mathbf{u}^{\prime}, \tilde{T}\right), G_{3, F}\left(\mathbf{u}^{\prime}, \tilde{S}\right)\right)^{\mathrm{T}}
$$

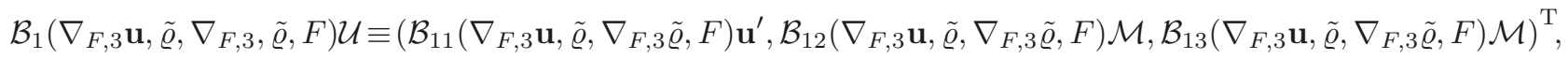

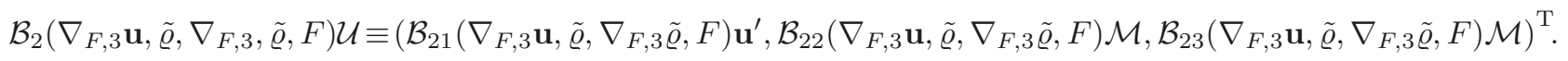
Note that $\mathbf{P}$ is calculated by the relationship

$$
\left\{\begin{array}{l}
a^{33}(F) \frac{\partial \tilde{p}^{(F)}}{\partial y_{3}}=-\varrho\left(\tilde{p}^{(F)}, \tilde{T}, \tilde{S}\right) g \quad \text { in } \Omega_{T_{1}}, \\
\left.\tilde{p}^{(F)}\right|_{y_{3}=F_{0}\left(y^{\prime}\right)}=p_{0} \quad \text { on } \mathbf{R}^{2},
\end{array}\right.
$$

which has a unique solution $\left(\tilde{p}^{(F)}, \tilde{T}, \tilde{S}\right)$ by virtue of the ordinary differential equation if $\tilde{p}^{(F)}$ has sufficient regularity. For $F$, taking the horizontal divergence of $(3.2)_{1}$, and adding it with $(3.1)_{6}$ yields the following problem:

$$
\left\{\begin{aligned}
\frac{\partial F}{\partial t}-\mathcal{L}_{4, \mathcal{U}, F} F= & q_{w}-\mathbf{u} \cdot \nabla F+u_{3}+\mu_{1} \sum_{i=1}^{2} \frac{\partial}{\partial y_{i}}\left(\nabla_{F} u_{i}\right) \cdot \nabla F \\
& -\sum_{i=1}^{2} \frac{\partial}{\partial y_{i}}\left(\mu_{2}\left(\nabla_{F, 3} \mathbf{u}, \tilde{\varrho}, \nabla_{F, 3} \tilde{\varrho}, F\right) a^{33} \frac{\partial u_{i}}{\partial y_{3}}\right)-\nabla \cdot\left(q_{w} \mathbf{u}\right)-\nabla \cdot \tau_{1} \quad \text { in } \mathbf{R}_{T_{1}}^{2} \\
\left.F\right|_{t=0}=F_{0} \quad \text { on } \mathbf{R}^{2} &
\end{aligned}\right.
$$

where $\mathcal{L}_{4, \mathcal{U}, F} F \equiv \mu_{1} \sum_{i=1}^{2} \nabla_{F} u_{i} \cdot \nabla \frac{\partial F}{\partial y_{i}}$. As we have stated, the problem from (3.7)-(3.9) now constitutes a problem for $\left(\mathbf{u}^{\prime}, \tilde{T}, \tilde{S}, F, \mathbf{P}\right)$.

\subsection{Statement of main result}

Hereafter, we denote the value of $p$ at $t=0$ by $p_{u}(x)$, which satisfies the following relationship:

$$
p_{u}(x)=p_{0}\left(x^{\prime}, 0\right)+g \int_{F_{0}\left(x^{\prime}\right)}^{x_{3}} \varrho\left(\left(p_{u}, T_{0}, S_{0}\right)\left(x^{\prime}, z_{3}, 0\right)\right) \mathrm{d} z_{3} .
$$

We also introduce notations $\varrho_{u} \equiv \varrho\left(p_{u}, T_{0}, S_{0}\right), \mathcal{R}_{0} \equiv \mathcal{R}\left(\frac{\partial \mathbf{v}_{0}}{\partial x_{3}}, \varrho_{u}, \frac{\partial \varrho_{u}}{\partial x_{3}}\right)$. 
In addition, we define the following notations:

$$
\begin{aligned}
\mathcal{V}_{0} & \equiv\left(p_{u}, T_{0}, S_{0}\right)^{\mathrm{T}}, \quad \mu_{i 0}=\mu_{i}\left(\frac{\partial \mathbf{v}_{0}}{\partial y_{3}}, \varrho_{u}, \frac{\partial \varrho_{u}}{\partial y_{3}}\right)(i=2,4,6), \\
\mathcal{L}_{j, 0} & \equiv \mathcal{L}_{j, \mathcal{U}_{0}, F_{0}}=\mu_{2 j-1} \nabla^{2}+\mu_{2 j}\left(\frac{\partial \mathbf{v}_{0}}{\partial y_{3}}, \varrho_{u}, \frac{\partial \varrho_{u}}{\partial y_{3}}\right) \frac{\partial^{2}}{\partial y_{3}^{2}} \quad(j=1,2,3),
\end{aligned}
$$

where $\mathcal{U}_{0}=\left(\mathbf{v}_{0}, T_{0}, S_{0}\right)^{\mathrm{T}}$. Then, the main result of this paper concerning the small-time solvability of (3.7)-(3.9), which will be proved in Section 6, is provided as follows:

Theorem 3.1. Let $l \in(1 / 2,1)$, and $T_{1}$ be an arbitrary positive number. Assume that

(i) $\quad\left(\mathbf{v}_{0}, T_{0}, S_{0}\right) \in \mathcal{W}_{0} \equiv \bar{W}_{2, c}^{3+l}(\Omega) \times\left(\bar{W}_{2}^{2+l}(\Omega)\right)^{2}, F_{0} \in W_{2}^{\frac{5}{2}+l}\left(\mathbf{R}^{2}\right), 0<c_{u} \leq \min \left\{\left(1+\alpha_{i} \mathcal{R}_{0}\right)\left|\frac{\partial \mathbf{v}_{0}}{\partial x_{3}}\right|,\left|\frac{\partial \mathbf{v}_{0}}{\partial x_{3}}\right|\right\}$ $(i=2,4,6), \underline{T}_{0} \leq T_{0}(x)<\infty$, and $0<\underline{S}_{0} \leq S_{0}(x)<\infty$ with positive constants $c_{u}, \underline{T}_{0}$ and $\underline{S}_{0}$, respectively;

(ii) $b \in \bar{W}_{2}^{\frac{5}{2}+l}\left(\mathbf{R}^{2}\right)$ and $F_{0}\left(x^{\prime}\right)-b\left(x^{\prime}\right)>c_{0}>0$ on $\mathbf{R}^{2}$ with a positive constant $c_{0}$;

(iii) $\theta \in C^{3+\beta}\left(\mathbf{R}^{3}\right)$ with $1 / 2(l-1 / 2)<\beta<l+1 / 2$;

(iv) With a constant $l^{\prime \prime}>l, \tau_{i} \in \bar{W}_{2}^{\frac{3}{2}+l^{\prime \prime}, \frac{3}{4}+\frac{l^{\prime \prime}}{2}}\left(\mathbf{R}_{T_{1}}^{2}\right)(i=1,2,3), q_{w} \in W_{2}^{\frac{3}{2}+l^{\prime \prime}, \frac{3}{4}+\frac{l^{\prime \prime}}{2}}\left(\mathbf{R}_{T_{1}}^{2}\right),\left.\frac{\partial \mathbf{v}_{0}}{\partial y_{i}}\right|_{\Gamma} \in W_{2}^{\frac{3}{2}+l^{\prime \prime}}\left(\mathbf{R}^{2}\right)$, $\frac{\partial p_{0}}{\partial y_{i}} \in W_{2}^{\frac{3}{2}+l^{\prime \prime}, \frac{3}{4}+\frac{l^{\prime \prime}}{2}}\left(\mathbf{R}_{T_{1}}^{2}\right), p_{0} \in \bar{W}_{2}^{\frac{5}{2}+l, \frac{5}{4}+\frac{l}{2}}\left(\mathbf{R}_{T_{1}}^{2}\right)$. It is also assumed that for the case of $|\alpha|=3$ with a multi-index $\alpha, D_{x}^{\alpha} p_{0}$ satisfy the Hölder condition with exponent $\beta_{p_{0}}>l / 2$ with respect to $x_{3}$;

(v) $\frac{\partial v_{10}}{\partial x_{1}} \frac{\partial v_{20}}{\partial x_{2}}-\frac{\partial v_{10}}{\partial x_{2}} \frac{\partial v_{20}}{\partial x_{1}}(x)>0 \quad \forall x \in \Omega$;

$$
\begin{aligned}
& M_{0}(x) \equiv \mathbf{v}_{0} \cdot \nabla \mathbf{v}_{0}-\left(\mathbf{v}_{0} \cdot \nabla b+\int_{b\left(x^{\prime}\right)}^{x_{3}} \nabla \cdot \mathbf{v}_{0}\left(x^{\prime}, z_{3}\right) \mathrm{d} z_{3}\right) \frac{\partial \mathbf{v}_{0}}{\partial x_{3}}-f \mathbf{A v}_{0} \in W_{2}^{1+l}(\Omega), \\
& \left.M_{1}\left(x^{\prime}\right) \equiv \tau_{1}\right|_{t=0}+\left.\mu_{20} \frac{\partial \mathbf{v}_{0}}{\partial x_{3}}\right|_{\Gamma} \in W_{2}^{\frac{3}{2}+l}\left(\mathbf{R}^{2}\right), \\
& \left.M_{2}\left(x^{\prime}\right) \equiv \tau_{2}\right|_{t=0}-\left.\mu_{40} \theta_{x}\left(\mathcal{V}_{0}\right) \varrho\left(\mathcal{V}_{0}\right) g\right|_{\Gamma} \in W_{2}^{\frac{3}{2}+l}\left(\mathbf{R}^{2}\right), \\
& \left.M_{3}\left(x^{\prime}\right) \equiv \tau_{3}\right|_{t=0}-\left.\mu_{20} \theta_{x}\left(\mathcal{V}_{0}\right) \varrho\left(\mathcal{V}_{0}\right) g\right|_{\Gamma_{b}} \in W_{2}^{\frac{3}{2}+l}\left(\mathbf{R}^{2}\right), \\
& M_{4}\left(x^{\prime}\right) \equiv \int_{b\left(x^{\prime}\right)}^{F_{0}\left(x^{\prime}\right)} \nabla \cdot \mathbf{v}_{0}\left(x^{\prime}, z_{3}\right) \mathrm{d} z_{3}+\sum_{i=1}^{2} \frac{\partial \mu_{20}}{\partial y_{i}} \frac{\partial \mathbf{v}_{0}}{\partial y_{3}} \in W_{2}^{\frac{1}{2}+l}\left(\mathbf{R}^{2}\right) ;
\end{aligned}
$$

(vii) $\varrho \in C^{4+\beta^{\prime}}(\mathcal{G})$ on $\mathcal{G}=\left\{x=\left(x_{1}, x_{2}, x_{3}\right) \in \mathbf{R}^{3} \mid x_{1}>c_{1}, x_{2}>\underline{T}_{0}, x_{3}>\underline{S}_{0}\right\}$ with $l / 2<\beta^{\prime}<1+l / 2$, $\inf _{x \in \mathcal{G}} \varrho(x) \geq c_{1}>0$ and $\sup _{x \in \mathcal{G}}\left|D^{\alpha} \varrho(x)\right| \leq M_{\varrho}$ for $|\alpha| \leq 4$.

Moreover, the compatibility conditions up to the order $1[32,48]$ are satisfied. Then, there exists $T^{*} \in\left(0, T_{1}\right]$ such that the problem (3.7)-(3.9) has a unique solution

$$
(\mathcal{U}, \mathbf{P}, F) \in \mathcal{W}\left(T^{*}\right) \equiv W_{2}^{3+l, \frac{3+l}{2}}\left(\Omega_{T^{*}}\right) \times\left(\bar{W}_{2}^{3+l, \frac{3+l}{2}}\left(\Omega_{T^{*}}\right)\right)^{2} \times W_{2}^{\frac{3}{2}+l, \frac{3}{4}+\frac{l}{2}}\left(\Omega_{T^{*}}\right) \times W_{2}^{\frac{5}{2}+l, \frac{5}{4}+\frac{l}{2}}\left(\mathbf{R}_{T^{*}}^{2}\right)
$$

satisfying $0<\frac{\underline{T}_{0}}{2} \leq \tilde{T}<\infty$ and $0<\frac{\underline{S}_{0}}{2} \leq \tilde{S}<\infty$ on $\Omega_{T^{*}}$. 


\subsection{Overview of proof of Theorem 3.1}

In the following sections, we provide the proof of Theorem 3.1 in several steps. Here we provide an overview of the proof [50].

The next section is devoted to auxiliary lemmas, which will be used later in considering the linear and nonlinear problems. Then, we consider the linear problems for $\mathcal{U}$ and $F$ in Section 5 . There, coefficients of the principal terms and the right-hand side of each problem are assumed to be provided with sufficient regularity. For the problem of $\mathcal{U}$, we first consider the coefficients independent of time, then the original one. This process is achieved by the regularizer method described in the same section. In Section 6, we consider the nonlinear problem by using the iteration method. A sequence of unknown variables is recursively constructed, and we deduce the boundedness of the sequence first by virtue of the results of the linear problem. Next, we show that the sequence forms a Cauchy sequence, which converges to the desired solution. This is achieved owing to Lemmas 4.10 and 6.3. The uniqueness of this solution is proved easily in a similar manner, and we omit it. These are shown to hold on a sufficiently short time interval. Finally, we show that $\tilde{T}$ and $\tilde{S}$ are bounded from below on a sufficiently short time interval. This completes the proof of Theorem 3.1.

\section{Auxiliary lemmas}

In this section, we prepare some lemmas used in the following sections. We first prepare lemmas concerning multiplicative inequalities in Sobolev-Slobodetskil spaces. Lemmas 4.1-4.2 hold on

$$
\Omega_{T_{1}}=\left\{(y, t)=\left(y^{\prime}, y_{3}, t\right) \mid y^{\prime} \in \mathbf{R}^{2}, b\left(y^{\prime}\right) \leq y_{3} \leq F_{0}\left(y^{\prime}\right), t \in\left(0, T_{1}\right]\right\} .
$$

Lemma 4.1. For $T_{1}>0$ arbitrarily provided and functions $f \in W_{2}^{\frac{3}{2}+l, \frac{3}{4}+\frac{l}{2}}\left(\mathbf{R}_{T_{1}}^{2}\right), g_{11}, g_{12} \in W_{2}^{1+l, \frac{1+l}{2}}\left(\Omega_{T_{1}}\right)$, and $g_{2} \in W_{2}^{2+l, \frac{2+l}{2}}\left(\Omega_{T_{1}}\right)$, in general, the following estimates hold:

$$
\begin{aligned}
\left\|f g_{11}\right\|_{W_{2}^{1+l, \frac{1+l}{2}}\left(\Omega_{T_{1}}\right)} & \leq C_{1}\|f\|_{W_{2}^{\frac{3}{2}+l, \frac{3}{4}+\frac{l}{2}}\left(\mathbf{R}_{T_{1}}^{2}\right)}\left\|g_{11}\right\|_{W_{2}^{1+l, \frac{1+l}{2}}}\left(\Omega_{T_{1}}\right)^{\prime} \\
\left\|f g_{2}\right\|_{W_{2}^{\frac{3}{2}+l, \frac{3}{4}+\frac{l}{2}}\left(\Omega_{T_{1}}\right)} & \leq C_{2}\|f\|_{W_{2}^{\frac{3}{2}+l, \frac{3}{4}+\frac{l}{2}}\left(\mathbf{R}_{T_{1}}^{2}\right)}\left\|g_{2}\right\|_{W_{2}^{2+l, \frac{2+l}{2}}}\left(\Omega_{T_{1}}\right)^{\prime} \\
\left\|f g_{11} g_{12}\right\|_{W_{2}^{l, \frac{l}{2}}}\left(\Omega_{T_{1}}\right) & \leq C_{3}\|f\|_{W_{2}^{\frac{3}{2}+l, \frac{3}{4}+\frac{l}{2}}\left(\mathbf{R}_{T_{1}}^{2}\right)}\left\|g_{11}\right\|_{W_{2}^{1+l, \frac{1+l}{2}}}\left(\Omega_{T_{1}}\right)
\end{aligned}
$$

with positive constants $C_{i}(i=1,2,3)$ depending on $\|b\|_{\bar{W}_{2}^{\frac{5}{2}+l}\left(\mathbf{R}^{2}\right)}$ and $\left\|F_{0}\right\|_{W_{2}^{\frac{5}{2}+l}\left(\mathbf{R}^{2}\right)}$.

The proof of this lemma is similar to that of Lemma 4.3 in [26], and we omit it here. Hereafter, $C$ stands for positive constants depending on $\|b\|_{\bar{W}_{2}^{\frac{5}{2}+l}\left(\mathbf{R}^{2}\right)},\left\|F_{0}\right\|_{W_{2}^{\frac{5}{2}+l}\left(\mathbf{R}^{2}\right)}$, and $\left\|p_{0}\right\|_{\bar{W}_{2}^{\frac{5}{2}+l, \frac{4}{5}+\frac{l}{2}}\left(\mathbf{R}_{T_{1}}^{2}\right)}$, and $\phi(\cdot)$ for increasing positive functions of their arguments.

Lemma 4.2. For $T_{1}>0$ arbitrarily provided and function $f \in W_{2}^{l, \frac{l}{2}}\left(\Omega_{T_{1}}\right)$ in general, we have the following estimate:

$$
\left\|\frac{f}{b-F_{0}}\right\|_{W_{2}^{l, \frac{l}{2}}\left(\Omega_{T_{1}}\right)}^{2} \leq C\|f\|_{W_{2}^{l, \frac{l}{2}}\left(\Omega_{T_{1}}\right)}^{2} .
$$

For Lemmas 4.3 and 4.5-4.7, below, we assume the following assumption: a number $T_{1}>0$ and a function $F_{*}$ satisfy $F_{*} \in W_{2}^{\frac{5}{2}+l, \frac{5}{4}+\frac{l}{2}}\left(\mathbf{R}_{T_{1}}^{2}\right)$ and $F_{*}\left(y^{\prime}, t\right)-b\left(y^{\prime}\right)>c_{0}$ for $t \in\left[0, T_{1}\right]$, and $F_{*}\left(y^{\prime}, 0\right)=F_{0}\left(y^{\prime}\right)$. We shall call this assumption $\left(A_{F_{*}}\right)$. The coordinate transform (1.15) is executed with $F$ replaced by $F_{*}$. The functions $b$ and $F_{0}$ are assumed to satisfy the assumptions imposed in the statement of Theorem 3.1, that is, $b \in \bar{W}_{2}^{\frac{5}{2}+l}\left(\mathbf{R}^{2}\right)$, $F_{0} \in W_{2}^{\frac{5}{2}+l}\left(\mathbf{R}^{2}\right)$ and $F_{0}\left(y^{\prime}\right)-b\left(y^{\prime}\right)>c_{0}$. The following lemma is proved with some elementary calculations, and we introduce it without proof. 
Lemma 4.3. For $T_{1}>0, F_{*} \in W_{2}^{\frac{5}{2}+l, \frac{5}{4}+\frac{l}{2}}\left(\mathbf{R}_{T_{1}}^{2}\right)$ and function $f \in \bar{W}_{2}^{3+l, \frac{3+l}{2}}\left(\mathbf{R}_{T_{1}}^{3}\right)$ in general, we have the following estimate:

$$
\begin{aligned}
\phi\left(\left\|F_{*}\right\|_{W_{2}^{i-\frac{1}{2}+l, \frac{i}{2}-\frac{1}{4}+\frac{l}{2}}\left(\mathbf{R}_{T_{1}}^{2}\right)}\right)\|f\|_{W_{2}^{i+l, \frac{i+l}{2}}\left(\mathbf{R}_{T_{1}}^{3}\right)} & \leq\left\|\tilde{f}^{\left(F_{*}\right)}\right\|_{W_{2}^{i+l, \frac{i+l}{2}}\left(\Omega_{T_{1}}\right)} \\
& \leq C \phi\left(\left\|F_{*}\right\|_{W_{2}^{i-\frac{1}{2}+l, \frac{i}{2}-\frac{1}{4}+\frac{l}{2}}\left(\mathbf{R}_{T_{1}}^{2}\right)}\right)\|f\|_{W_{2}^{i+l, \frac{i+l}{2}}\left(\mathbf{R}_{T_{1}}^{3}\right)}(i=2,3) .
\end{aligned}
$$

For the estimate of $p$, we prepare the following lemma:

Lemma 4.4. For arbitrary $l>1 / 2$ and $F_{0} \in W_{2}^{\frac{5}{2}+l}\left(\mathbf{R}^{2}\right)$, let us introduce a notation of an interval

$$
I_{b, F_{0}}=\left(-\sup _{\mathbf{R}^{2}}|b|, \sup _{\mathbf{R}^{2}}\left|F_{0}\right|\right)
$$

and let function $g=g\left(y, z_{3}\right)$, defined on $\Omega \times I_{b, F_{0}}$, satisfy

$$
\int_{I_{b, F_{0}}}\left\|g\left(\cdot, z_{3}\right)\right\|_{\dot{W}_{2}^{l-\frac{1}{2}}(\Omega)}^{2} \mathrm{~d} z_{3}+\int_{\Omega}\left|g\left(y, z_{3}\right)\right|^{2} \mathrm{~d} y<\infty \quad \forall z_{3} \in I_{b, F_{0}} .
$$

We also introduce a function defined on $\Omega, \Phi_{g}(y) \equiv \int_{F_{0}\left(y^{\prime}\right)}^{y_{3}} g\left(y, z_{3}\right) \mathrm{d} z_{3}$. Then, we have the following estimate with a certain $y_{30} \in I_{b, F_{0}}$ :

$$
\left\|\Phi_{g}\right\|_{\dot{W}_{2}^{l-\frac{1}{2}}(\Omega)}^{2} \leq C\left\{\int_{I_{b, F_{0}}}\left\|g\left(\cdot, z_{3}\right)\right\|_{\dot{W}_{2}^{l-\frac{1}{2}}(\Omega)}^{2} \mathrm{~d} z_{3}+\int_{\Omega}\left|g\left(y, y_{30}\right)\right|^{2} \mathrm{~d} y\right\} .
$$

Similarly, if we take function $f \in W_{2}^{l}(\Omega)$ and define function $\Phi_{f}(y) \equiv \int_{F_{0}\left(y^{\prime}\right)}^{y_{3}} f\left(y^{\prime}, z_{3}\right) \mathrm{d} z_{3}$, defined on $\Omega$, the following estimate holds:

$$
\left\|\Phi_{f}\right\|_{\dot{W}_{2}^{l}(\Omega)}^{2} \leq C\|f\|_{W_{2}^{l}(\Omega)}^{2} .
$$

Proof. The proofs of both inequalities are similar, so we only show the first one. For two points, $y^{(i)}=$ $\left(y^{(i) \prime}, y_{3}^{(i)}\right) \in \Omega(i=1,2)$, the following inequality holds:

$$
\begin{aligned}
\left|\Phi_{g}\left(y^{(1)}\right)-\Phi_{g}\left(y^{(2)}\right)\right|^{2} \leq & 3\left\{\left|\int_{F_{0}\left(y^{(1) \prime}\right)}^{y_{3}^{(1)}} g\left(y^{(1)}, z_{3}\right) \mathrm{d} z_{3}-\int_{F_{0}\left(y^{(1) \prime}\right)}^{y_{3}^{(2)}} g\left(y^{(1)}, z_{3}\right) \mathrm{d} z_{3}\right|^{2}\right. \\
& +\left|\int_{F_{0}\left(y^{(1) \prime}\right)}^{y_{3}^{(2)}} g\left(y^{(1)}, z_{3}\right) \mathrm{d} z_{3}-\int_{F_{0}\left(y^{(2) \prime}\right)}^{y_{3}^{(2)}} g\left(y^{(1)}, z_{3}\right) \mathrm{d} z_{3}\right|^{2} \\
& \left.+\left|\int_{F_{0}\left(y^{(2) \prime}\right)}^{y_{3}^{(2)}}\left\{g\left(y^{(1)}, z_{3}\right)-g\left(y^{(2)}, z_{3}\right)\right\} \mathrm{d} z_{3}\right|^{2}\right\} \equiv 3 \sum_{i=1}^{3} I_{i} .
\end{aligned}
$$

In accordance with the mean value theorem, there exists a certain $y_{30} \in I_{b, F_{0}}$, such that

$$
\int_{\Omega} \int_{\Omega} \frac{I_{1}}{\left|y^{(1)}-y^{(2)}\right|^{2+2 l}} \mathrm{~d} y^{(1)} \mathrm{d} y^{(2)} \leq \int_{\Omega} \int_{\Omega} \frac{\left|y_{3}^{(1)}-y_{3}^{(2)}\right|^{2}\left|g\left(y^{(1)}, y_{30}\right)\right|^{2}}{\left|y^{(1)}-y^{(2)}\right|^{2+2 l}} \mathrm{~d} y^{(1)} \mathrm{d} y^{(2)} .
$$


Letting $\tilde{y}=\left(\tilde{y}^{\prime}, \tilde{y}_{3}\right) \equiv y^{(1)}-y^{(2)}$, it is easily seen that

$$
\int_{\mathbf{R}^{2}} \frac{\left|y_{3}^{(1)}-y_{3}^{(2)}\right|^{2}}{|\tilde{y}|^{2+2 l}} \mathrm{~d} \tilde{y}^{\prime}=\left|\tilde{y}_{3}\right|^{2-2 l} \int_{0}^{\infty} \frac{\mathrm{d} \chi}{(1+\chi)^{\frac{2+2 l}{2}}}
$$

and therefore,

$$
\int_{\Omega} \int_{\Omega} \frac{I_{1}}{\left|y^{(1)}-y^{(2)}\right|^{2+2 l}} \mathrm{~d} y^{(1)} \mathrm{d} y^{(2)} \leq C \int_{\Omega}\left|g\left(y^{(1)}, y_{30}\right)\right|^{2} \mathrm{~d} y^{(1)}
$$

Next, we have

$$
\begin{aligned}
& \int_{\Omega} \int_{\Omega} \frac{I_{2}}{\left|y^{(1)}-y^{(2)}\right|^{2+2 l}} \mathrm{~d} y^{(1)} \mathrm{d} y^{(2)} \\
& \leq \int_{\Omega} \int_{\Omega} \frac{\left|F_{0}\left(y^{(1) \prime}\right)-F_{0}\left(y^{(2) \prime}\right)\right| \int_{I_{g}}\left|g\left(y^{(1)}, z_{3}\right)\right|^{2} \mathrm{~d} z_{3}}{\left|y^{(1)}-y^{(2)}\right|^{2+2 l}} \mathrm{~d} y^{(1)} \mathrm{d} y^{(2)} \\
& \leq \int_{I_{g}}\left\|g\left(\cdot, z_{3}\right)\right\|_{L_{2}(\Omega)}^{2} \mathrm{~d} z_{3} \int_{\Omega} \frac{\left|F_{0}\left(y^{(1) \prime}\right)-F_{0}\left(y^{(1) \prime}-\tilde{y}^{\prime}\right)\right|}{|\tilde{y}|^{2+2 l}} \mathrm{~d} \tilde{y} .
\end{aligned}
$$

Then, noting the fact $W_{2}^{\frac{5}{2}+l}\left(\mathbf{R}^{2}\right) \subset C^{\frac{3}{2}+l}\left(\mathbf{R}^{2}\right)$,

$$
\int_{\Omega} \frac{\left|F_{0}\left(y^{(1) \prime}\right)-F_{0}\left(y^{(1) \prime}-\tilde{y}^{\prime}\right)\right|}{|\tilde{y}|^{2+2 l}} \mathrm{~d} \tilde{y} \leq C\left\{\int_{|\tilde{y}| \leq 1} \frac{\left|\tilde{y}^{\prime}\right|^{\frac{3}{2}+l}}{|\tilde{y}|^{2+2 l}} \mathrm{~d} \tilde{y}+\int_{|\tilde{y}|>1} \frac{\left|\tilde{y}^{\prime}\right|}{|\tilde{y}|^{2+2 l}} \mathrm{~d} \tilde{y}\right\}<\infty .
$$

The estimate of $I_{3}$ is obtained in a similar manner, and we arrive at the first statement. The second statement (4.1) is proved more easily, and we omit the proof of it here.

The next lemma enables the estimate of $F$ later.

Lemma 4.5. For arbitrary $T_{1}>0, F_{*} \in W_{2}^{\frac{5}{2}+\frac{l}{2}, \frac{5}{4}+\frac{l}{2}}\left(\mathbf{R}_{T_{1}}^{2}\right)$, such that $\left.F_{*}\right|_{t=0}=F_{0} \in W_{2}^{\frac{5}{2}+l}\left(\mathbf{R}^{2}\right), \mathbf{w}=\mathbf{w}^{\prime}+\tilde{\mathbf{v}}_{0}^{\left(F_{*}\right)}$, with $\tilde{\mathbf{v}}_{0}^{\left(F_{*}\right)}$ being defined earlier, the following estimates hold:

$$
\left\|\nabla_{F_{*}} \cdot \mathbf{w}\right\|_{W_{2}^{i+l, \frac{i+l}{2}}\left(\Omega_{T_{1}}\right)}^{2} \leq\left(\phi_{4 i}\left(\left\|F_{*}\right\|_{W_{2}^{\frac{5}{2}+l, \frac{5}{4}+\frac{l}{2}}\left(\mathbf{R}_{T_{1}}^{2}\right)}\right)+C\right)\|\mathbf{w}\|_{W_{2}^{i+l, \frac{i+l}{2}}\left(\Omega_{T_{1}}\right)}^{2}(i=1,2),
$$

where $\phi_{4 i}(\cdot)(i=1,2)$ stand for homogeneous polynomials of their arguments, with coefficients depending on $\|b\|_{\bar{W}_{2}^{\frac{5}{2}+l}\left(\mathbf{R}^{2}\right)}$ and $\left\|F_{0}\right\|_{W_{2}^{\frac{5}{2}+l}\left(\mathbf{R}^{2}\right)}$.

In addition, the following lemma is useful in the iteration process in the final section.

Lemma 4.6. For arbitrary $T_{1}>0, F_{*} \in W_{2}^{\frac{5}{2}+l, \frac{5}{4}+\frac{l}{2}}\left(\mathbf{R}_{T_{1}}^{2}\right)$ such that $\left.F_{*}\right|_{t=0}=F_{0} \in W_{2}^{\frac{5}{2}+l}\left(\mathbf{R}^{2}\right)$,

$$
\left\|\tilde{\mathbf{v}}_{0}^{\left(F_{*}\right)}-\mathbf{v}_{0}\right\|_{W_{2}^{i+l, \frac{i+l}{2}}\left(\Omega_{T_{1}}\right)} \leq\left(\epsilon+\epsilon T_{1}\right)\left\|F_{*}-F_{0}\right\|_{W_{2}^{\frac{5}{2}+l, \frac{5}{4}+\frac{l}{2}}\left(\mathbf{R}_{T_{1}}^{2}\right)}\left\|\mathbf{v}_{0}\right\|_{\bar{W}_{2, c}^{3+l}(\Omega)} \quad(i=1,2)
$$

holds with a small positive constant $\epsilon$ and $C_{\epsilon}$ depending on $\epsilon$. 
Proof. By applying the mean value theorem,

$$
\begin{aligned}
\tilde{\mathbf{v}}_{0}^{\left(F_{*}\right)}-\mathbf{v}_{0} & =\overline{\mathbf{v}}_{0}\left(y^{\prime}, X_{3}^{\left(F_{*}\right)}(y, t)\right)-\mathbf{v}_{0}(y) \\
& =\int_{0}^{1} \frac{\mathrm{d}}{\mathrm{d} \chi} \overline{\mathbf{v}}_{0}\left(y^{\prime}, \chi X_{3}^{\left(F_{*}\right)}(y, t)+(1-\chi) y_{3}\right) \mathrm{d} \chi \\
& =\frac{\left(F_{*}\left(y^{\prime}, t\right)-F_{0}\left(y^{\prime}\right)\right)\left(b\left(y^{\prime}\right)-y_{3}\right)}{b\left(y^{\prime}\right)-F_{0}\left(y^{\prime}\right)} \int_{0}^{1} \frac{\partial \mathbf{v}_{0}}{\partial y_{3}}\left(y^{\prime}, \chi X_{3}^{\left(F_{*}\right)}(y, t)+(1-\chi) y_{3}\right) \mathrm{d} \chi .
\end{aligned}
$$

This, and by applying the interpolation inequality of Sobolev-Slobodetskil spaces and the estimate of the extended function, lead to the desired result.

Now, we show some estimates concerning the derivatives of $\tilde{p}^{(F)}$.

Lemma 4.7. For $T_{1}>0, p_{0} \in \bar{W}_{2}^{\frac{5}{2}+l, \frac{5}{4}+\frac{l}{2}}\left(\mathbf{R}_{T_{1}}^{2}\right)$ and $F_{*} \in W_{2}^{\frac{5}{2}+l, \frac{5}{4}+\frac{l}{2}}\left(\mathbf{R}_{T_{1}}^{2}\right)$ provided, which satisfies $\left.F_{*}\right|_{t=0}=$ $F_{0} \in W_{2}^{\frac{5}{2}+l}\left(\mathbf{R}^{2}\right)$, the following estimates hold:

$$
\left\|D_{y}^{\alpha} \tilde{p}^{\left(F_{*}\right)}\right\|_{W_{2}^{\frac{5}{2}-|\alpha|+l, \frac{\frac{5}{2}-|\alpha|+l}{2}}\left(\Omega_{T_{1}}\right)} \leq \phi\left(\|\mathcal{M}\|_{W_{2}^{|\alpha|+l, \frac{|\alpha|+l}{2}}\left(\Omega_{T_{1}}\right)}\right)\left\|F_{*}\right\|_{W_{2}^{\frac{5}{2}+l, \frac{5}{4}+\frac{l}{2}}\left(\Omega_{T_{1}}\right)} \quad(|\alpha|=1,2,3),
$$

where $\mathcal{M}=(\tilde{T}, \tilde{S})^{\mathrm{T}}$.

Proof. We first show the following equalities with $i, j, k=1,2$ :

$$
\begin{aligned}
\frac{\partial \tilde{p}^{\left(F_{*}\right)}}{\partial y_{i}} & =\frac{\partial p_{0}}{\partial y_{i}} \Phi_{1}^{\left(\mathcal{M}, F_{*}\right)}+\frac{g}{a_{*}^{33}} \int_{F_{0}\left(y^{\prime}\right)}^{y_{3}} \Psi_{1}^{\left(\mathcal{M}, F_{*}\right)}\left(y^{\prime}, z_{3}, t\right) \Phi_{2}^{\left(\mathcal{M}, F_{*}\right)}\left(y, z_{3}, t\right) \mathrm{d} z_{3}, \\
\frac{\partial^{2} \tilde{p}^{\left(F_{*}\right)}}{\partial y_{i} \partial y_{j}} & =\frac{\partial^{2} p_{0}}{\partial y_{i} \partial y_{j}} \Phi_{1}^{\left(\mathcal{M}, F_{*}\right)}+\frac{g}{a_{*}^{33}} \int_{F_{0}\left(y^{\prime}\right)}^{y_{3}} \Psi_{2}^{\left(\mathcal{M}, F_{*}\right)}\left(y^{\prime}, z_{3}, t\right) \Phi_{2}^{\left(\mathcal{M}, F_{*}\right)}\left(y, z_{3}, t\right) \mathrm{d} z_{3}, \\
\frac{\partial^{3} \tilde{p}^{\left(F_{*}\right)}}{\partial y_{i} \partial y_{j} \partial y_{k}} & =\frac{\partial^{3} p_{0}}{\partial y_{i} \partial y_{j} \partial y_{k}} \Phi_{1}^{\left(\mathcal{M}, F_{*}\right)}+\frac{g}{a_{*}^{33}} \int_{F_{0}\left(y^{\prime}\right)}^{y_{3}} \Psi_{3}^{\left(\mathcal{M}, F_{*}\right)}\left(y^{\prime}, z_{3}, t\right) \Phi_{2}^{\left(\mathcal{M}, F_{*}\right)}\left(y, z_{3}, t\right) \mathrm{d} z_{3},
\end{aligned}
$$

where

$$
\begin{aligned}
\Phi_{1}^{\left(\mathcal{M}, F_{*}\right)}(y, t) & =\exp \left(-\frac{g}{a_{*}^{33}} \int_{F_{0}\left(y^{\prime}\right)}^{y_{3}} \frac{\partial \varrho}{\partial z_{1}}\left(\mathcal{V}\left(y^{\prime}, z_{3}, t\right)\right) \mathrm{d} z_{3}\right) \\
\Phi_{2}^{\left(\mathcal{M}, F_{*}\right)}\left(y, z_{3}, t\right) & =\exp \left(-\frac{g}{a_{*}^{33}} \int_{z_{3}}^{y_{3}} \frac{\partial \varrho}{\partial z_{1}}\left(\mathcal{V}\left(y^{\prime}, \tau, t\right)\right) \mathrm{d} \tau\right) \\
\Psi_{1}^{\left(\mathcal{M}, F_{*}\right)}(y, t) & =-\frac{g}{a_{*}^{33}}\left(\frac{\partial \varrho}{\partial z_{2}} \frac{\partial \tilde{T}}{\partial y_{i}}+\frac{\partial \varrho}{\partial z_{3}} \frac{\partial \tilde{S}}{\partial y_{i}}\right)+\frac{\partial}{\partial y_{i}}\left(\frac{g}{a_{*}^{33}}\right) \varrho(\mathcal{V}(y, t)) \equiv \Psi_{11}^{\left(\mathcal{M}, F_{*}\right)}+\Psi_{12}^{\left(\mathcal{M}, F_{*}\right)}, \\
\Psi_{2}^{\left(\mathcal{M}, F_{*}\right)}(y, t) & =-\frac{\partial}{\partial y_{j}}\left(\frac{g}{a_{*}^{33}} \frac{\partial \varrho}{\partial z_{1}}\right) \frac{\partial \tilde{p}^{\left(F_{*}\right)}}{\partial y_{i}}+\frac{\partial \Psi_{1}^{\left(\mathcal{M}, F_{*}\right)}}{\partial y_{j}}, \\
\Psi_{3}^{\left(\mathcal{M}, F_{*}\right)}(y, t) & =-\frac{\partial}{\partial y_{k}}\left(\frac{g}{a_{*}^{33}} \frac{\partial \varrho}{\partial z_{1}}\right) \frac{\partial^{2} \tilde{p}^{\left(F_{*}\right)}}{\partial y_{i} \partial y_{j}}+\frac{\partial \Psi_{2}^{\left(\mathcal{M}, F_{*}\right)}}{\partial y_{k}}
\end{aligned}
$$


with $a_{*}^{33}=a^{33}\left(F_{*}\right)$. Actually, the differentiation of the hydrostatic relationship with respect to $y_{i}(i=1,2)$ leads to

$$
\frac{\partial^{2} \tilde{p}^{\left(F_{*}\right)}}{\partial y_{3} \partial y_{i}}=-\frac{g}{a_{*}^{33}} \frac{\partial \varrho}{\partial z_{1}} \frac{\partial \tilde{p}^{\left(F_{*}\right)}}{\partial y_{i}}+\Psi_{1}^{\left(\mathcal{M}, F_{*}\right)} .
$$

By solving this ordinary differential equation with respect to $y_{3}$ with the boundary condition

$$
\left.\frac{\partial \tilde{p}^{\left(F_{*}\right)}}{\partial y_{i}}\right|_{y_{3}=F_{0}\left(y^{\prime}\right)}=\frac{\partial p_{0}}{\partial y_{i}}
$$

we have

$$
\begin{aligned}
\frac{\partial \tilde{p}^{\left(F_{*}\right)}}{\partial y_{i}}= & \frac{\partial p_{0}}{\partial y_{i}} \exp \left(-\frac{g}{a_{*}^{33}} \int_{F_{0}\left(y^{\prime}\right)}^{y_{3}} \frac{\partial \varrho}{\partial z_{1}}\left(\mathcal{V}\left(y^{\prime}, z_{3}, t\right)\right) \mathrm{d} z_{3}\right) \\
& +\int_{F_{0}\left(y^{\prime}\right)}^{y_{3}} \Psi_{1}^{\left(\mathcal{M}, F_{*}\right)}\left(y^{\prime}, z_{3}, t\right) \exp \left(-\frac{g}{a_{*}^{33}} \int_{z_{3}}^{y_{3}} \frac{\partial \varrho}{\partial z_{1}}\left(\mathcal{V}\left(y^{\prime}, \tau, t\right)\right) \mathrm{d} \tau\right) \mathrm{d} z_{3} .
\end{aligned}
$$

Thus we have (4.2). (4.3) and (4.4) are shown in a similar manner. By applying Lemma 4.4, we have the desired result.

Now let us state a lemma concerning the differentiation of $p$ with different $F$. Let $\tilde{p}_{(i)}$ satisfy

$$
\tilde{p}_{(i)}(y, t)=p_{0}\left(y^{\prime}, t\right)+\frac{g}{a_{(i)}^{33}\left(y^{\prime}, t\right)} \int_{y_{3}}^{F_{0}\left(y^{\prime}\right)} \varrho\left(\mathcal{V}_{(i)}\left(y^{\prime}, z_{3}, t\right)\right) \mathrm{d} z_{3} \quad(i=1,2),
$$

where $\mathcal{V}_{(i)} \equiv\left(\tilde{p}_{(i)}, \tilde{T}_{(i)}, \tilde{S}_{(i)}\right)^{\mathrm{T}}=\left(\tilde{p}_{(i)}, \mathcal{M}_{(i)}\right)^{\mathrm{T}}, a_{(i)}^{33}=a^{33}\left(F_{(i)}\right)(i=1,2)$. In addition, we introduce notations

$$
\tilde{\mathcal{V}} \equiv \mathcal{V}_{(2)}-\mathcal{V}_{(1)} \equiv(\tilde{\tilde{p}}, \tilde{\tilde{T}}, \tilde{\tilde{S}})^{\mathrm{T}}, \widetilde{F} \equiv F_{(2)}-F_{(1)}, \widetilde{\mathcal{M}} \equiv(\tilde{\tilde{T}}, \tilde{\tilde{S}})^{\mathrm{T}}
$$

Based on these notations, we have the following lemma concerning the estimates of the derivatives of $\tilde{\tilde{p}}$ :

Lemma 4.8. For $T_{1}>0$, the following estimates hold:

$$
\left\|D_{y}^{\alpha} \tilde{\tilde{p}}\right\|_{W_{2}^{\frac{5}{2}-|\alpha|+l, \frac{5}{4}-\frac{|\alpha|}{2}+\frac{l}{2}}\left(\Omega_{T_{1}}\right)} \leq \phi_{0}\left(\|\widetilde{F}\|_{W_{2}^{|\alpha|+l-\frac{1}{2}+\frac{|\alpha|+l-1}{2}}\left(\Omega_{T_{1}}\right)},\|\widetilde{\mathcal{M}}\|_{W_{2}^{|\alpha|+l, \frac{|\alpha|+l}{2}}\left(\Omega_{T_{1}}\right)}\right)
$$

where $\phi_{0}(\cdot)$ is a homogeneous polynomial of its argument.

Proof. We first show the following estimates for $y, y^{(i)}(i=1,2) \in \Omega$ :

$$
\begin{gathered}
|\tilde{\tilde{p}}(y, t)| \leq \phi_{0}(|\widetilde{F}(y, t)|,|\widetilde{\mathcal{M}}(y, t)|), \\
\left|\tilde{\tilde{p}}\left(y^{(1)}, t\right)-\tilde{\tilde{p}}\left(y^{(2)}, t\right)\right| \leq \phi_{0}\left(\left|\widetilde{F}\left(y^{(1) \prime}, t\right)-\widetilde{F}\left(y^{(2) \prime}, t\right)\right|,\left|\widetilde{\mathcal{M}}\left(y^{(1)}, t\right)-\widetilde{\mathcal{M}}\left(y^{(2)}, t\right)\right|\right) .
\end{gathered}
$$

To see these, we take the difference of the equalities

$$
a_{(i)}^{33} \frac{\partial \tilde{p}^{\left(F_{(i)}\right)}}{\partial y_{3}}=-g \varrho\left(\mathcal{V}_{(i)}\right) \quad(i=1,2),
$$


which yields

$$
a_{(2)}^{33} \frac{\partial \tilde{\tilde{p}}}{\partial y_{3}}=\frac{g \widetilde{F}}{b-F_{0}} \frac{\partial \tilde{p}\left(F_{(1)}\right)}{\partial y_{3}}-g \widetilde{\mathcal{V}} \cdot \int_{0}^{1} \nabla_{3} \varrho\left(\mathcal{V}_{\sigma^{\prime}}\right) \mathrm{d} \sigma^{\prime},
$$

where $\mathcal{V}_{\sigma^{\prime}}=\sigma^{\prime} \mathcal{V}_{(1)}+\left(1-\sigma^{\prime}\right) \mathcal{V}_{(2)}$. The boundary condition is provided by

$$
\left.\tilde{\tilde{p}}\right|_{y_{3}=F_{0}\left(y^{\prime}\right)}=0 .
$$

From the classical result of the ordinary differential equation, we then have

$$
\begin{aligned}
\tilde{\tilde{p}}(y, t)= & \int_{F_{0}}^{y_{3}}\left\{\frac{g \widetilde{F}}{b-F_{0}} \frac{\partial \tilde{p}^{\left(F_{(1)}\right)}}{\partial y_{3}}\left(y^{\prime}, z_{3}, t\right)-g\left(\int_{0}^{1} \nabla_{2} \varrho\left(\mathcal{V}_{\sigma^{\prime}}\left(y^{\prime}, z_{3}, t\right)\right) \mathrm{d} \sigma^{\prime}\right) \cdot \widetilde{\mathcal{M}}\left(y^{\prime}, z_{3}, t\right)\right\} \\
& \times \exp \left(-g \int_{z_{3}}^{y_{3}} \int_{0}^{1} \frac{\partial \varrho}{\partial z_{1}}\left(\mathcal{V}_{\sigma^{\prime}}\left(y^{\prime}, \tau, t\right)\right) \mathrm{d} \sigma^{\prime} \mathrm{d} \tau\right) \mathrm{d} z_{3},
\end{aligned}
$$

where $\nabla_{2} \varrho=\left(\frac{\partial \varrho}{\partial z_{2}}, \frac{\partial \varrho}{\partial z_{3}}\right)^{\mathrm{T}}$. From this, (4.5)-(4.6) are easily obtained. Due to (4.2), on the other hand, we obtain the explicit form of $\frac{\partial \tilde{\tilde{p}}}{\partial y_{i}}(i=1,2)$ by making use of the proof of Lemma 4.7 :

$$
\begin{aligned}
\frac{\partial \tilde{\tilde{p}}}{\partial y_{i}}= & \frac{\partial p_{0}}{\partial y_{i}}\left\{\exp \left(-\frac{g}{a_{(1)}^{33}} \int_{F_{0}\left(y^{\prime}\right)}^{y_{3}} \frac{\partial \varrho}{\partial z_{1}}\left(\mathcal{V}_{(1)}\left(y^{\prime}, z_{3}, t\right)\right) \mathrm{d} z_{3}\right)\right. \\
& \left.-\exp \left(-\frac{g}{a_{(2)}^{33}} \int_{F_{0}\left(y^{\prime}\right)}^{y_{3}} \frac{\partial \varrho}{\partial z_{1}}\left(\mathcal{V}_{(2)}\left(y^{\prime}, z_{3}, t\right)\right) \mathrm{d} z_{3}\right)\right\} \\
& +\int_{F_{0}\left(y^{\prime}\right)}^{y_{3}}\left(\Psi_{1}^{(1)}-\Psi_{1}^{(2)}\right) \exp \left(-\frac{g}{a_{(1)}^{33}} \int_{z_{3}}^{y_{3}} \frac{\partial \varrho}{\partial z_{1}}\left(\mathcal{V}_{(1)}\left(y^{\prime}, \tau, t\right)\right) \mathrm{d} \tau\right) \mathrm{d} z_{3} \\
& +\int_{F_{0}\left(y^{\prime}\right)}^{y_{3}} \Psi_{1}^{(2)}\left\{\exp \left(-\frac{g}{a_{(1)}^{33}} \int_{z_{3}}^{y_{3}} \frac{\partial \varrho}{\partial z_{1}}\left(\mathcal{V}_{(1)}\left(y^{\prime}, \tau, t\right)\right) \mathrm{d} \tau\right)\right. \\
& \left.-\exp \left(-\frac{g}{a_{(2)}^{33}} \int_{z_{3}}^{y_{3}} \frac{\partial \varrho}{\partial z_{1}}\left(\mathcal{V}_{(2)}\left(y^{\prime}, \tau, t\right)\right) \mathrm{d} \tau\right)\right\} \equiv \sum_{i=1}^{3} \bar{K}_{i},
\end{aligned}
$$

where $\Psi_{1}^{(k)} \equiv \Psi_{1}^{\left(\mathcal{M}_{(k)}, F_{(k)}\right)}(k=1,2)$.

By virtue of the mean value theorem, the first term in the right-hand side of (4.7) is described as follows:

$$
\begin{aligned}
\bar{K}_{1}= & \left\{-\frac{g \widetilde{F}}{b-F_{0}} \int_{F_{0}\left(y^{\prime}\right)}^{y_{3}} \frac{\partial \varrho}{\partial z_{1}}\left(\mathcal{V}_{(1)}\left(y^{\prime}, z_{3}, t\right)\right) \mathrm{d} z_{3}\right. \\
& \left.+\frac{g}{a_{(1)}^{33}} \int_{F_{0}}^{y_{3}} \int_{0}^{1} \nabla_{3} \frac{\partial \varrho}{\partial z_{1}}\left(\mathcal{V}_{\sigma}\left(y^{\prime}, z_{3}, t\right)\right) \cdot \widetilde{\mathcal{V}}\left(y^{\prime}, z_{3}, t\right) \mathrm{d} \sigma \mathrm{d} z_{3}\right\} \int_{0}^{1} \exp \left(J_{\sigma^{\prime}}\right) \mathrm{d} \sigma^{\prime},
\end{aligned}
$$

where

$$
J_{\sigma^{\prime}}=-\frac{\sigma^{\prime} g}{a_{(1)}^{33}} \int_{F_{0}\left(y^{\prime}\right)}^{y_{3}} \frac{\partial \varrho}{\partial z_{1}}\left(\mathcal{V}_{(1)}\left(y^{\prime}, z_{3}, t\right)\right) \mathrm{d} z_{3}-\frac{\left(1-\sigma^{\prime}\right) g}{a_{(2)}^{33}} \int_{F_{0}\left(y^{\prime}\right)}^{y_{3}} \frac{\partial \varrho}{\partial z_{1}}\left(\mathcal{V}_{(2)}\left(y^{\prime}, z_{3}, t\right)\right) \mathrm{d} z_{3}
$$


Next, it is easily seen that

$$
\begin{aligned}
\Psi_{11}^{(1)}-\Psi_{11}^{(2)}= & -\frac{g \widetilde{F}}{b-F_{0}}\left(\nabla_{2} \cdot \frac{\partial \mathcal{M}}{\partial y_{i}}\right)-\frac{g}{a_{(2)}^{33}} \nabla_{2} \varrho\left(\mathcal{V}_{(2)}\right) \cdot \frac{\partial \widetilde{\mathcal{M}}}{\partial y_{i}} \\
& -\frac{g \widetilde{\mathcal{V}}}{a_{(2)}^{33}}\left\{\left(\int_{0}^{1} \nabla_{3} \frac{\partial \varrho}{\partial z_{2}}\left(\mathcal{V}_{\sigma}\right) \mathrm{d} \sigma\right) \frac{\partial \tilde{T}}{\partial y_{i}}+\left(\int_{0}^{1} \nabla_{3} \frac{\partial \varrho}{\partial z_{3}}\left(\mathcal{V}_{\sigma}\right) \mathrm{d} \sigma\right) \frac{\partial \tilde{S}}{\partial y_{i}}\right\}, \\
\Psi_{12}^{(1)}-\Psi_{12}^{(2)}= & -\frac{\partial}{\partial y_{i}}\left(\frac{g \widetilde{F}}{b-F_{0}}\right) \varrho\left(\mathcal{V}_{(1)}\right)+\frac{\partial}{\partial y_{i}}\left(\frac{g}{a_{(2)}^{33}}\right)\left(\int_{0}^{1} \nabla_{3} \varrho\left(\mathcal{V}_{\sigma}\right) \mathrm{d} \sigma\right) \tilde{\mathcal{V}},
\end{aligned}
$$

where $\Psi_{1 j}^{(i)}=\Psi_{1 j}^{\left(\mathcal{M}_{(i)}, F_{(i)}\right)}(i, j=1,2)$. Applying (4.5) and (4.6) together with these, it is possible to estimate $\left\|\frac{\partial \tilde{\tilde{p}}}{\partial y_{i}}\right\|_{\dot{W}_{2}^{l}(\Omega)}$, and we have the desired result with the help of Lemma 4.2. Other cases are calculated in a similar manner.

For arbitrary $T_{1}>0$, we introduce new notations of function spaces:

$$
\begin{gathered}
\mathcal{W}_{1}\left(T_{1}\right) \equiv\left(W_{2}^{1+l, \frac{1+l}{2}}\left(\Omega_{T_{1}}\right)\right)^{3}, \quad \mathcal{W}_{2}\left(T_{1}\right) \equiv\left(W_{2}^{\frac{3}{2}+l, \frac{3}{4}+\frac{l}{2}}\left(\Gamma_{T_{1}}\right)\right)^{3}, \quad \mathcal{W}_{3}\left(T_{1}\right) \equiv\left(W_{2}^{\frac{3}{2}+l, \frac{3}{4}+\frac{l}{2}}\left(\Gamma_{b T_{1}}\right)\right)^{3} \\
\mathcal{W}_{\mathcal{H}}\left(T_{1}\right) \equiv \prod_{i=1}^{3} \mathcal{W}_{i}\left(T_{1}\right) .
\end{gathered}
$$

The next lemma is concerned with the estimate of the differential of a function with respect to time in Sobolev space [49]. It is useful in estimating the difference of the operators with respect to time in Lemma 4.10.

Lemma 4.9. For arbitrary $T_{1}>0$ and function $g \in W_{2}^{1+l, \frac{1+l}{2}}\left(\Omega_{T_{1}}\right)$ in general, there exists a positive constant $\beta_{1}$, such that

$$
\|g(\cdot, t)-g(\cdot, 0)\|_{W_{2}^{l}(\Omega)}^{2} \leq C t^{\beta_{1}}\|g\|_{W_{2}^{l+2 \beta_{1}, \frac{l}{2}+\beta_{1}}\left(\Omega_{T_{1}}\right)}^{2}
$$

holds in the time interval $\left(0, T_{1}\right)$.

Proof. First, we extend the function $g$ onto the space $\mathbf{R}^{3} \times(0, \infty)$ preserving the regularity [54], so that what we discuss in the following is based on the assumption $g \in W_{2}^{1+l, \frac{1+l}{2}}\left(\mathbf{R}^{3} \times(0, \infty)\right)$ without loss of generality. From the elementary fact of the spacial Fourier transform, the following representation holds:

$$
\|g(\cdot, t)-g(\cdot, 0)\|_{W_{2}^{l}\left(\mathbf{R}^{3}\right)}^{2}=\int_{\mathbf{R}^{3}}\left(1+|\xi|^{2}\right)^{l}\left|\mathcal{F}_{y}[g(y, t)-g(y, 0)](\xi, t)\right|^{2} \mathrm{~d} \xi,
$$

where $\mathcal{F}_{y}[f]$ stands for the Fourier transform of function $f(y)$ with respect to $y$. On the other hand, by virtue of the inverse Fourier transform with respect to time, the following relationship holds:

$$
g(y, t+\tau)-g(y, \tau)=\frac{1}{2 \pi} \int_{\mathbf{R}} \exp (i \tau \eta)(\exp (i t \eta)-1) \hat{g}^{t}(y, \eta) \mathrm{d} \eta
$$


where $\hat{g}^{t}(y, \eta)$ is the Fourier transform of $g$ with respect to time. By taking $\tau=0$ in (4.9), and then substituting it into (4.8), we have

$$
\begin{aligned}
\|g(\cdot, t)-g(\cdot, 0)\|_{W_{2}^{l}\left(\mathbf{R}^{3}\right)}^{2} & =\frac{1}{2 \pi} \int_{\mathbf{R}^{3}}\left(1+|\xi|^{2}\right)^{l}\left|\mathcal{F}_{y}\left[\int_{\mathbf{R}}(\exp (i t \eta)-1) \hat{g}^{t}(y, \eta) \mathrm{d} \eta\right](\xi, t)\right|^{2} \mathrm{~d} \xi \\
& =\frac{1}{2 \pi} \int_{\mathbf{R}^{3}}\left(1+|\xi|^{2}\right)^{l}\left|\int_{\mathbf{R}}(\exp (i t \eta)-1) \hat{g}^{y, t}(\xi, \eta) \mathrm{d} \eta\right|^{2} \mathrm{~d} \xi \\
& \leq\left\{\int_{\mathbf{R}}\left(\int_{\mathbf{R}^{3}}\left(1+|\xi|^{2}\right)^{l}(\exp (i t \eta)-1)^{2}\left|\hat{g}^{y, t}(\xi, \eta)\right|^{2} \mathrm{~d} \xi\right)^{\frac{1}{2}} \mathrm{~d} \eta\right\}^{2} \\
& =\int_{\mathbf{R}}(\exp (i t \eta)-1)^{2}\left\|\hat{g}^{t}(\eta)\right\|_{W_{2}^{l}\left(\mathbf{R}^{3}\right)}^{2} \mathrm{~d} \eta \\
& \leq C t^{\beta_{1}} \int_{\mathbf{R}}\left(1+\eta^{2}\right)^{\beta_{1}}\left\|\hat{g}^{t}(\eta)\right\|_{W_{2}^{l}\left(\mathbf{R}^{3}\right)}^{2} \mathrm{~d} \eta \leq C t^{\beta_{1}}\|g\|_{W_{2}^{l+2 \beta_{1}, \frac{l}{2}+\beta_{1}}\left(\mathbf{R}^{3} \times(0, \infty)\right)}^{2}
\end{aligned}
$$

In (4.10), $\hat{g}^{y, t}$ means the Fourier transform with respect to both space and time, and we have applied Minkowski's inequality in the first inequality. This and the way of extension completes the proof.

Now, we can prove the following lemma, which plays an important role in later:

Lemma 4.10. For $T_{1}>0$ small enough, define $\left(\mathbf{u}_{*}, \tilde{T}_{*}, \tilde{S}_{*}, F_{*}\right), \quad\left(\mathbf{u}_{(i)}, \tilde{T}_{(i)}, \tilde{S}_{(i)}, F_{(i)}\right) \in \bar{W}_{2, c}^{3+l, \frac{3+l}{2}}\left(\Omega_{T_{1}}\right) \times$ $\bar{W}_{2}^{3+l, \frac{3+l}{2}}\left(\Omega_{T_{1}}\right) \times \bar{W}_{2}^{3+l, \frac{3+l}{2}}\left(\Omega_{T_{1}}\right) \times W_{2}^{\frac{5}{2}+l, \frac{5}{4}+\frac{l}{2}}\left(\mathbf{R}_{T_{1}}^{2}\right)$ with

$$
\left.\left(\mathbf{u}_{*}, \tilde{T}_{*}, \tilde{S}_{*}, F_{*}\right)\right|_{t=0}=\left.\left(\mathbf{u}_{(i)}, \tilde{T}_{(i)}, \tilde{S}_{(i)}, F_{(i)}\right)\right|_{t=0}=\left(\mathbf{v}_{0}, T_{0}, S_{0}, F_{0}\right)(i=1,2) .
$$

That is, $\mathbf{u}_{*}=\mathbf{u}_{*}^{\prime}+\tilde{\mathbf{v}}_{0}^{\left(F_{*}\right)}, \mathbf{u}_{(i)}=\mathbf{u}_{(i)}^{\prime}+\tilde{\mathbf{v}}_{0}^{\left(F_{(i)}\right)}(i=1,2)$. Let $\tilde{p}_{*}, \tilde{\varrho}_{*}=\tilde{\varrho}_{*}(y, t) \equiv \varrho\left(\tilde{p}_{*}, \tilde{T}_{*}, \tilde{S}_{*}\right), \tilde{p}_{(i)}, \tilde{\varrho}_{(i)}=$ $\tilde{\varrho}_{(i)}(y, t) \equiv \varrho\left(\tilde{p}_{(i)}, \tilde{T}_{(i)}, \tilde{S}_{(i)}\right)(i=1,2)$ satisfy

$$
\begin{aligned}
a_{*}^{33} \frac{\partial \tilde{p}_{*}}{\partial y_{3}} & =-g \tilde{\varrho}_{*}, \text { in } \Omega_{T_{1}},\left.\quad \tilde{p}_{*}\right|_{y_{3}=F_{0}\left(y^{\prime}\right)}=p_{0}\left(y^{\prime}, t\right) \text { on } \Gamma_{T_{1}}, \\
a_{(i)}^{33} \frac{\partial \tilde{p}_{(i)}}{\partial y_{3}} & =-g \tilde{\varrho}_{(i)}, \text { in } \Omega_{T_{1}},\left.\quad \tilde{p}_{(i)}\right|_{y_{3}=F_{0}\left(y^{\prime}\right)}=p_{0}\left(y^{\prime}, t\right) \text { on } \Gamma_{T_{1}}(i=1,2),
\end{aligned}
$$

respectively, where $a_{*}^{33}=a^{33}\left(F_{*}\right)$ and $a_{(i)}^{33}=a^{33}\left(F_{(i)}\right)$. We also assume $\left(\mathbf{v}_{0}, T_{0}, S_{0}\right) \in \mathcal{W}_{0}, F_{0} \in W_{2}^{\frac{5}{2}+l}\left(\mathbf{R}^{2}\right)$, $\varrho \in C^{4+\beta^{\prime}}(\mathcal{G})$ on $\mathcal{G}=\left\{x=\left(x_{1}, x_{2}, x_{3}\right) \in \mathbf{R}^{3} \mid x_{1}>c_{1}, x_{2}>\underline{T}_{0}, x_{3}>\underline{S}_{0}\right\}$ with $l / 2<\beta^{\prime}<1+l / 2$. Let us introduce notations

$$
\begin{gathered}
\mathcal{W}_{\mathcal{U}}\left(T_{1}\right) \equiv W_{2}^{3+l, \frac{3+l}{2}}\left(\Omega_{T_{1}}\right) \times \bar{W}_{2}^{3+l, \frac{3+l}{2}}\left(\Omega_{T_{1}}\right) \times \bar{W}_{2}^{3+l, \frac{3+l}{2}}\left(\Omega_{T_{1}}\right), \mathcal{W}_{\mathcal{U}}^{\prime}\left(T_{1}\right) \equiv\left(W_{2}^{3+l, \frac{3+l}{2}}\left(\Omega_{T_{1}}\right)\right)^{3}, \\
\mathcal{U}_{(i)}=\left(\mathbf{u}_{(i)}^{\prime}, \tilde{T}_{(i)}, \tilde{S}_{(i)}\right)^{\mathrm{T}}, \quad \mathcal{U}_{*}=\left(\mathbf{u}_{*}^{\prime}, \tilde{T}_{*}, \tilde{S}_{*}\right)^{\mathrm{T}}, \quad \tilde{\mathcal{U}}=\left(\mathbf{u}_{(1)}-\mathbf{u}_{(2)}, \tilde{T}_{(1)}-\tilde{T}_{(2)}, \tilde{S}_{(1)}-\tilde{S}_{(2)}\right)^{\mathrm{T}}, \\
\mathcal{L}_{0} \mathcal{U}=\left(\mathcal{L}_{1,0} \mathbf{u}^{\prime}, \mathcal{L}_{2,0} \tilde{T}, \mathcal{L}_{3,0} \tilde{S}\right)^{\mathrm{T}}, \quad \mathcal{B}_{i, 0}=\mathcal{B}_{i}\left(\frac{\partial \mathbf{v}_{0}}{\partial y_{3}}, \varrho_{u}, \frac{\partial \varrho_{u}}{\partial y_{3}}, F_{0}\right), \mathcal{B}_{i(*)} \equiv \mathcal{B}_{i}\left(\nabla_{F_{*}, 3} \mathbf{u}_{*}, \tilde{\varrho}_{*}, \nabla_{F_{*}, 3} \tilde{\varrho}_{*}\right), \\
\mathcal{B}_{i(j)} \equiv \mathcal{B}_{i}\left(\nabla_{F_{(j)}, 3} \mathbf{u}_{(j)}, \tilde{\varrho}_{(j)}, \nabla_{F_{(j)}, 3} \tilde{\varrho}_{(j)}\right)(i, j=1,2), \\
\mu_{2(i)} \equiv \mu_{2 a}\left(1+\alpha_{2} \mathcal{R}_{(i)}\right)^{-2}+\mu_{2 b}(i=1,2), \mu_{m(i)} \equiv \mu_{m a}\left(1+\alpha_{m} \mathcal{R}_{(i)}\right)^{-1}+\mu_{m b}(i=1,2, m=4,6),
\end{gathered}
$$


H. HONDA

where $\mathcal{R}_{(i)} \equiv \frac{g \tilde{\varrho}_{(i)}^{-1}}{a_{(i)}^{33}} \frac{\partial \tilde{\varrho}_{(i)}}{\partial y_{3}}\left|\frac{\partial \mathbf{u}_{(i)}}{\partial y_{3}}\right|^{-2}(i=1,2)$. We also define $\mathcal{R}_{*} \equiv \frac{g \tilde{\varrho}_{*}^{-1}}{a_{*}^{33}} \frac{\partial \tilde{Q}_{*}}{\partial y_{3}}\left|\frac{\partial \mathbf{u}_{*}}{\partial y_{3}}\right|^{-2}, \mathcal{V}_{*} \equiv\left(\tilde{p}_{*}, \tilde{T}_{*}, \tilde{S}_{*}\right)^{\mathrm{T}}$ and assume $\left(1+\alpha_{i} \mathcal{R}_{0}\right)\left|\frac{\partial \mathbf{v}_{0}}{\partial y_{3}}\right| \geq c_{u}>0(i=2,4,6)$. Let $T_{1}>0$ and a function $F_{*}$ satisfy $F_{*} \in W_{2}^{\frac{5}{2}+l, \frac{5}{4}+\frac{l}{2}}\left(\mathbf{R}_{T_{1}}^{2}\right)$, $F_{*}\left(y^{\prime}, t\right)-b\left(y^{\prime}\right)>c_{0}, \min \left\{\left(1+\alpha_{i} \mathcal{R}_{*}\right)\left|\frac{\partial \mathbf{u}_{*}}{\partial y_{3}}\right|,\left|\frac{\partial \mathbf{u}_{*}}{\partial y_{3}}\right|\right\} \geq c_{u}>0$ and $\min \left\{\left(1+\alpha_{i} \mathcal{R}_{(j)}\right)\left|\frac{\partial \mathbf{u}_{(j)}}{\partial y_{3}}\right|,\left|\frac{\partial \mathbf{u}_{(j)}}{\partial y_{3}}\right|\right\} \geq c_{u}>0$ $(i=2,4,6, j=1,2)$ for any $t \in\left(0, T_{1}\right]$. Then, under these assumptions, the following estimates hold for any $t \in\left(0, T_{1}\right]$ and $\epsilon>0$ :

$$
\begin{aligned}
&\left\|\mathcal{L}_{\mathcal{U}_{(1)}, F_{(1)}} \mathcal{U}_{*}\right\|_{\mathcal{W}_{1}(t)}+\sum_{i=1}^{2}\left\|\mathcal{B}_{i(1)} \mathcal{U}_{*}\right\|_{\mathcal{W}_{i+1}(t)} \\
& \leq \phi_{01}\left(\left\|F_{(1)}\right\|_{W_{2}^{\frac{5}{2}+l, \frac{5}{4}+\frac{l}{2}}\left(\mathbf{R}_{t}^{2}\right)},\left\|F_{*}\right\|_{W_{2}^{\frac{5}{2}+l, \frac{5}{4}+\frac{l}{2}}\left(\mathbf{R}_{t}^{2}\right)},\left\|\mathcal{U}_{(1)}\right\|_{\mathcal{W}_{\mathcal{U}}(t)},\left\|\mathcal{U}_{*}\right\|_{\mathcal{W}_{\mathcal{U}}(t)}\right) \\
&\left\|\left[\mathcal{L}_{\mathcal{U}_{(1)}, F_{(1)}}-\mathcal{L}_{\mathcal{U}_{(2)}, F_{(2)}}\right] \mathcal{U}_{*}\right\|_{\mathcal{W}_{1}(t)}+\sum_{i=1}^{2}\left\|\left[\mathcal{B}_{i(1)}-\mathcal{B}_{i(2)}\right] \mathcal{U}_{*}\right\|_{\mathcal{W}_{i+1}(t)} \\
& \leq\left(\epsilon+C_{\epsilon} t\right) \phi_{02}\left(\sum_{i=1}^{2}\left(\left\|F_{(i)}\right\|_{W_{2}^{\frac{5}{2}+l, \frac{5}{4}+\frac{l}{2}}\left(\mathbf{R}_{t}^{2}\right)}+\left\|\mathcal{U}_{(i)}\right\|_{\mathcal{W}_{\mathcal{U}}(t)}\right),\left\|\mathcal{U}_{*}\right\|_{\mathcal{W}_{\mathcal{U}}(t)}\right) \\
&\left\|\left[\mathcal{L}_{\mathcal{U}_{(1)}, F_{(1)}}-\mathcal{L}_{0}\right] \mathcal{U}_{*}\right\|_{\mathcal{W}_{1}(t)}+\sum_{i=1}^{2} \|\left[\tilde{\mathcal{U}}_{i(1)}-\mathcal{B}_{\mathcal{W}_{\mathcal{U}}^{\prime}(t)}+\left\|\widetilde{\mathcal{H}}_{*}\right\|_{\mathcal{W}_{i+1}(t)} \|_{W_{2}^{\frac{5}{2}+l, \frac{5}{4}+\frac{l}{2}}\left(\mathbf{R}_{t}^{2}\right)}\right) \\
& \leq\left(\epsilon+C_{\epsilon} t\right) \phi_{03}\left(\left\|F_{(1)}\right\|_{W_{2}^{\frac{5}{2}+l, \frac{5}{4}+\frac{l}{2}}\left(\mathbf{R}_{t}^{2}\right)},\left\|\mathcal{U}_{(1)}\right\|_{\mathcal{W}_{\mathcal{U}}(t)}\right)\left\|\mathcal{U}_{*}\right\|_{\mathcal{W}_{\mathcal{U}}(t)},
\end{aligned}
$$

where $\phi_{0 i}(\cdot)(i=1,2,3)$ are homogeneous polynomials of their arguments, and $C_{\epsilon}$ is a positive constant depending on $\epsilon$.

Proof. Without losing generality, we show the statement in the case of $t=T_{1}$. Since the inequality (4.13) is the most difficult to prove, we only show the essence of the proof of it here. (4.11) and (4.12) are proved in a similar manner, though more easily.

In the proof of (4.13), it is most important and very difficult to estimate the term

$$
\left\|\left(\mu_{4(1)}-\mu_{40}\right) \theta_{x_{1}}\left(\tilde{p}_{(1)}, \tilde{T}_{(1)}, \tilde{S}_{(1)}\right) a_{(1)}^{33} \frac{\partial \tilde{T}_{*}}{\partial y_{3}}\right\|_{W_{2}^{\frac{3}{2}+l, \frac{3}{4}+\frac{l}{2}}\left(\Gamma_{T_{1}}\right)} .
$$

Especially, we show the proof of the term

$$
\left\|\left(\frac{\partial^{2}}{\partial y_{i} \partial y_{j}}\left(\mu_{4(1)}-\mu_{40}\right)\right) \theta_{x_{1}}\left(\tilde{p}_{(1)}, \tilde{T}_{(1)}, \tilde{S}_{(1)}\right) a_{(1)}^{33} \frac{\partial \tilde{T}_{*}}{\partial y_{3}}\right\|_{W_{2}^{l-\frac{1}{2}, \frac{l}{2}-\frac{1}{4}}\left(\Gamma_{T_{1}}\right)},
$$

with $i, j=1,2$, which appears in (4.14). For the sake of simplicity, we denote $\left.f\right|_{\Gamma}=f\left(y^{\prime}, F_{0}\left(y^{\prime}\right), t\right)$ by $f\left(y^{\prime}, t\right)$ hereafter for function $f$. Expanding the term of the second order derivative in (4.15), the amount in the norm, 
denoted by $J$, is represented as follows:

$$
\begin{aligned}
J= & \left.\mu_{4 a}\left(1+\alpha_{4} \mathcal{R}_{(1)}\right)^{-2}\left|\frac{\partial \mathbf{u}_{(1)}}{\partial y_{3}}\right|^{-4}\left(\psi_{(1)} \cdot \frac{\partial^{2} \mathbf{u}_{(1)}}{\partial y_{i} \partial y_{3}}\right)\left(\psi_{(1)} \cdot \frac{\partial^{2} \mathbf{u}_{(1)}}{\partial y_{j} \partial y_{3}}\right)\right|_{\Gamma} \\
& -\left.\mu_{4 a}\left(1+\alpha_{4} \mathcal{R}_{0}\right)^{-2}\left|\frac{\partial \mathbf{u}_{0}}{\partial y_{3}}\right|^{-4}\left(\psi_{0} \cdot \frac{\partial^{2} \mathbf{u}_{0}}{\partial y_{i} \partial y_{3}}\right)\left(\psi_{0} \cdot \frac{\partial^{2} \mathbf{u}_{0}}{\partial y_{j} \partial y_{3}}\right)\right|_{\Gamma} \equiv \Theta-\Theta_{0},
\end{aligned}
$$

where

$$
\psi_{(1)}=\left.\left|\frac{\partial \mathbf{u}_{(1)}}{\partial y_{3}}\right|^{-1} \frac{\partial \mathbf{u}_{(1)}}{\partial y_{3}}\right|_{\Gamma}, \psi_{0}=\left.\left|\frac{\partial \mathbf{u}_{0}}{\partial y_{3}}\right|^{-1} \frac{\partial \mathbf{u}_{0}}{\partial y_{3}}\right|_{\Gamma} .
$$

Thanks to Lemma 4.9, we have the estimate with $0<\epsilon<1 / 2$ such that

$$
\left\|\Theta(\cdot, t)-\Theta_{0}(\cdot)\right\|_{W_{2}^{l-\frac{1}{2}}\left(\mathbf{R}^{2}\right)} \leq C t^{\frac{\epsilon}{2}}\|\Theta\|_{W_{2}^{l-\frac{1}{2}+\epsilon, \frac{l}{2}-\frac{1}{4}+\frac{\epsilon}{2}}\left(\mathbf{R}_{t}^{2}\right)} .
$$

Therefore, we only have to estimate the right-hand side of (4.16). First, we show the estimate of

$$
\|\Theta(t)\|_{\dot{W}_{2}^{l-\frac{1}{2}+\epsilon}\left(\mathbf{R}^{2}\right)}^{2} .
$$

Taking $\left|\psi_{(1)}\right|=1$ and the boundedness of the amount

$$
\left(1+\alpha_{4} \mathcal{R}_{(1)}\right)^{-2}\left|\frac{\partial \mathbf{u}_{(1)}}{\partial y_{3}}\right|^{-4}
$$

on a short time interval into account, it is essential to estimate the amount

$$
J_{1}(t) \equiv \int_{\mathbf{R}^{2}} \int_{\mathbf{R}^{2}} \frac{\left|\psi_{(1)}\left(y_{1}^{\prime}, t\right)-\psi_{(1)}\left(y_{2}^{\prime}, t\right)\right|^{2}\left|\frac{\partial^{2} \mathbf{u}_{(1)}}{\partial y_{i} \partial y_{3}}\left(y_{1}^{\prime}, t\right)\right|^{2}\left|\frac{\partial^{2} \mathbf{u}_{(1)}}{\partial y_{j} \partial y_{3}}\left(y_{1}^{\prime}, t\right)\right|^{2}}{\left|y_{1}^{\prime}-y_{2}^{\prime}\right|^{1+2 l+2 \epsilon}} \mathrm{d} y_{1}^{\prime} \mathrm{d} y_{2}^{\prime} .
$$

By taking $z^{\prime}=y_{1}^{\prime}-y_{2}^{\prime}$ in the right-hand side of the above, and taking the positive constant $\tau<\frac{2}{1-l}$, the Hölder's inequality with respect to $y_{1}^{\prime}$ and the Sobolev embedding theorem lead to

$$
J_{1}(t) \leq\left\|\frac{\partial^{2} \mathbf{u}_{(1)}}{\partial y_{i} \partial y_{3}}(t)\right\|_{L_{\tau}\left(\mathbf{R}^{2}\right)}^{2}\left\|\frac{\partial^{2} \mathbf{u}_{(1)}}{\partial y_{j} \partial y_{3}}(t)\right\|_{L_{\tau}\left(\mathbf{R}^{2}\right)}^{2} \int_{\mathbf{R}^{2}} \frac{\left\|\psi_{(1)}(\cdot, t)-\psi_{(1)}\left(\cdot-z^{\prime}, t\right)\right\|_{W_{2}^{1}\left(\mathbf{R}^{2}\right)}^{2}}{\left|z^{\prime}\right|^{1+2 l+2 \epsilon}} \mathrm{d} z^{\prime} .
$$

In order to estimate the term

$$
\int_{\mathbf{R}^{2}} \frac{\left\|\frac{\partial}{\partial y_{k}} \psi_{(1)}(\cdot, t)-\frac{\partial}{\partial y_{k}} \psi_{(1)}\left(\cdot-z^{\prime}, t\right)\right\|_{L_{2}\left(\mathbf{R}^{2}\right)}^{2}}{\left|z^{\prime}\right|^{1+2 l+2 \epsilon}} \mathrm{d} z^{\prime} \quad(k=1,2),
$$

we note the fact that

$$
\frac{\partial}{\partial y_{k}} \psi_{(1)}=\frac{\partial^{2} \mathbf{u}_{(1)}}{\partial y_{k} \partial y_{3}}\left|\frac{\partial \mathbf{u}_{(1)}}{\partial y_{3}}\right|^{-1}-\frac{\partial \mathbf{u}_{(1)}}{\partial y_{3}} \cdot \frac{\partial^{2} \mathbf{u}_{(1)}}{\partial y_{k} \partial y_{3}}\left|\frac{\partial \mathbf{u}_{(1)}}{\partial y_{3}}\right|^{-\frac{3}{2}} \frac{\partial \mathbf{u}_{(1)}}{\partial y_{3}},
$$

and the assumption (i) in Theorem 3.1. We estimate the term

$$
\begin{aligned}
& J_{2}(t) \equiv \\
& \qquad \int_{\mathbf{R}^{2}} \int_{\mathbf{R}^{2}} \frac{\left|\frac{\partial \mathbf{u}_{(1)}}{\partial y_{3}}\left(y_{1}^{\prime}, t\right) \cdot \frac{\partial^{2} \mathbf{u}_{(1)}}{\partial y_{k} \partial y_{3}}\left(y_{1}^{\prime}, t\right) \frac{\partial \mathbf{u}_{(1)}}{\partial y_{3}}\left(y_{1}^{\prime}, t\right)-\frac{\partial \mathbf{u}_{(1)}}{\partial y_{3}}\left(y_{1}^{\prime}-z^{\prime}, t\right) \cdot \frac{\partial^{2} \mathbf{u}_{(1)}}{\partial y_{k} \partial y_{3}}\left(y_{1}^{\prime}-z^{\prime}, t\right) \frac{\partial \mathbf{u}_{(1)}}{\partial y_{3}}\left(y_{1}^{\prime}-z^{\prime}, t\right)\right|^{2}}{\left|z^{\prime}\right|^{1+2 l+2 \epsilon}} \mathrm{d} z^{\prime} \mathrm{d} y_{1}^{\prime},
\end{aligned}
$$


which is essential in estimating (4.18). By virtue of Hölder's inequality and the Sobolev embedding theorem again, we have

$$
\begin{aligned}
J_{2}(t) & \leq 2 \sup _{\mathbf{R}_{T_{1}}^{2}}\left|\frac{\partial \mathbf{u}_{(1)}}{\partial y_{3}}\right|^{2}\left\|\frac{\partial^{2} \mathbf{u}_{(1)}}{\partial y_{k} \partial y_{3}}(t)\right\|_{W_{2}^{l-\frac{1}{2}}\left(\mathbf{R}^{2}\right)}^{2}\left\|\frac{\partial \mathbf{u}_{(1)}}{\partial y_{3}}(t)\right\|_{\dot{W}_{2}^{l+\frac{1}{2}+\epsilon}\left(\mathbf{R}^{2}\right)}^{2}+\sup _{\mathbf{R}_{T_{1}}^{2}}\left|\frac{\partial \mathbf{u}_{(1)}}{\partial y_{3}}\right|^{4}\left\|\frac{\partial^{2} \mathbf{u}_{(1)}}{\partial y_{k} \partial y_{3}}(t)\right\|_{W_{2}^{l-\frac{1}{2}+\epsilon}\left(\mathbf{R}^{2}\right)}^{2} \\
& \leq C\left(2 K_{1}(t)+K_{2}(t)\right),
\end{aligned}
$$

where

$$
\begin{aligned}
& K_{1}(t) \equiv\left(\left\|\frac{\partial \mathbf{u}_{(1)}^{\prime}}{\partial y_{3}}\right\|_{W_{2}^{\frac{3}{2}+l, \frac{3}{4}+\frac{l}{2}}\left(\mathbf{R}_{T_{1}}^{2}\right)}^{2}+\left\|\mathbf{u}_{0}\right\|_{\bar{W}_{2, c}^{3+l, \frac{3+l}{2}}\left(\Omega_{T_{1}}\right)}^{2}\right)^{2}\left(\left\|\frac{\partial^{2} \mathbf{u}_{(1)}^{\prime}}{\partial y_{k} \partial y_{3}}(t)\right\|_{W_{2}^{l-\frac{1}{2}}\left(\mathbf{R}^{2}\right)}^{2}+\left\|\mathbf{u}_{0}\right\|_{\bar{W}_{2, c}^{3+l, \frac{3+l}{2}}\left(\Omega_{T_{1}}\right)}^{2}\right), \\
& K_{2}(t) \equiv\left(\left\|\frac{\partial \mathbf{u}_{(1)}^{\prime}}{\partial y_{3}}\right\|_{W_{2}^{\frac{3}{2}+l, \frac{3}{4}+\frac{l}{2}}\left(\mathbf{R}_{T_{1}}^{2}\right)}^{4}+\left\|\mathbf{u}_{0}\right\|_{\bar{W}_{2, c}^{3+l, \frac{3+l}{2}}\left(\Omega_{T_{1}}\right)}^{4}\right)\left(\left\|\frac{\partial^{2} \mathbf{u}_{(1)}^{\prime}}{\partial y_{k} \partial y_{3}}(t)\right\|_{W_{2}^{l-\frac{1}{2}+\epsilon}\left(\mathbf{R}^{2}\right)}^{2}+\left\|\mathbf{u}_{0}\right\|_{\bar{W}_{2, c}^{3+l, \frac{3+l}{2}}\left(\Omega_{T_{1}}\right)}^{2}\right) .
\end{aligned}
$$

Combining the discussions after (4.17), we have

$$
J_{1}(t) \leq C\left(2 K_{1}(t)+K_{2}(t)\right)\left\|\frac{\partial^{2} \mathbf{u}_{(1)}}{\partial y_{i} \partial y_{3}}(t)\right\|_{L_{\tau}\left(\mathbf{R}^{2}\right)}^{2}\left\|\frac{\partial^{2} \mathbf{u}_{(1)}}{\partial y_{j} \partial y_{3}}(t)\right\|_{L_{\tau}\left(\mathbf{R}^{2}\right)}^{2} .
$$

Next, we estimate $\int_{0}^{T_{1}} J_{1}(t) \mathrm{d} t$, which amounts to estimating $\int_{0}^{T_{1}}\|\Theta(t)\|_{\dot{W}_{2}^{l-\frac{1}{2}+\epsilon}\left(\mathbf{R}^{2}\right)}^{2} \mathrm{~d} t$. Owing to (4.19) and the representations of $K_{m}(m=1,2)$, it is sufficient to estimate the amount

$$
J_{3} \equiv \int_{0}^{T_{1}}\left\|\frac{\partial^{2} \mathbf{u}_{(1)}}{\partial y_{i} \partial y_{3}}(t)\right\|_{L_{\tau}\left(\mathbf{R}^{2}\right)}^{2}\left\|\frac{\partial^{2} \mathbf{u}_{(1)}}{\partial y_{j} \partial y_{3}}(t)\right\|_{L_{\tau}\left(\mathbf{R}^{2}\right)}^{2}\left\|\frac{\partial^{2} \mathbf{u}_{(1)}^{\prime}}{\partial y_{k} \partial y_{3}}(t)\right\|_{W_{2}^{l-\frac{1}{2}}\left(\mathbf{R}^{2}\right)}^{2} \mathrm{~d} t .
$$

Again, by virtue of Hölder's inequality, the Sobolev embedding theorem, and the multiplicative inequality of Sobolev-Slobodetskiu spaces, and by making use of the trace theorem and $\tau<\frac{2}{1-l}$, we arrive at the estimate

$$
\begin{aligned}
J_{3} & \leq \sup _{t \in\left(0, T_{1}\right)}\left\|\frac{\partial^{2} \mathbf{u}_{(1)}}{\partial y_{k} \partial y_{3}}\right\|_{W_{2}^{l-\frac{1}{2}}\left(\mathbf{R}^{2}\right)}\left\|\frac{\partial^{2} \mathbf{u}_{(1)}}{\partial y_{i} \partial y_{3}}\right\|_{L_{4}\left(0, T_{1} ; L_{\tau}\left(\mathbf{R}^{2}\right)\right)}^{2}\left\|\frac{\partial^{2} \mathbf{u}_{(1)}^{\prime}}{\partial y_{j} \partial y_{3}}\right\|_{L_{4}\left(0, T_{1} ; L_{\tau}\left(\mathbf{R}^{2}\right)\right)}^{2} \\
& \leq\left(\epsilon^{\prime}+C_{\epsilon^{\prime}} T_{1}\right)\left\|\mathbf{u}_{(1)}\right\|_{W_{2}^{3+l, \frac{3+l}{2}}\left(\Omega_{T_{1}}\right)}^{2}\left\|\frac{\partial^{2} \mathbf{u}_{(1)}}{\partial y_{i} \partial y_{3}}\right\|_{W_{2}^{1+l, \frac{1+l}{2}}\left(\Omega_{T_{1}}\right)}^{2}\left\|\frac{\partial^{2} \mathbf{u}_{(1)}^{\prime}}{\partial y_{j} \partial y_{3}}\right\|_{W_{2}^{1+l, \frac{1+l}{2}}\left(\Omega_{T_{1}}\right)}^{2},
\end{aligned}
$$

where $\epsilon^{\prime}>0$ is a small positive constant. This completes the estimate of $\|\Theta\|_{L_{2}\left(0, T_{1} ; \dot{W}_{2}^{l-\frac{1}{2}+\epsilon}\left(\mathbf{R}^{2}\right)\right)}^{2}$. The estimate of the time derivative of $\Theta$ is obtained by similar calculations, which leads to

$$
\|\Theta\|_{L_{2}\left(\mathbf{R}^{2} ; \dot{W}_{2}^{\frac{l}{2}-\frac{1}{4}+\frac{\epsilon}{2}}\left(0, T_{1}\right)\right)}^{2} \leq c\left(T_{1}\right)\left(1+\left\|\mathbf{u}_{(1)}^{\prime}\right\|_{W_{2}^{3+l, \frac{3+l}{2}}\left(\Omega_{T_{1}}\right)}\right)^{2}\left\|\mathbf{u}_{(1)}^{\prime}\right\|_{W_{2}^{3+l, \frac{3+l}{2}}}^{6}\left(\Omega_{T_{1}}\right),
$$

with an increasing function of time $c(\cdot)$, which tends to zero as $t$ does. These, together with (4.19) and by applying the multiplicative inequality to (4.15), yields the desired estimate (4.13). Estimates (4.11) and (4.12) are obtained in the same line, but more easily, and we omit the proofs here. 


\section{LinEAR PROBLEMS}

In this section, we consider linear problems for $\mathcal{U} \equiv\left(\mathbf{u}^{\prime}, \mathcal{M}\right)^{\mathrm{T}} \equiv\left(\mathbf{u}^{\prime}, \tilde{T}, \tilde{S}\right)^{\mathrm{T}}$ and $F$. Each problem will contribute to constructing the successive approximation of the nonlinear problem in the next section.

\subsection{Extension of initial data into the region $t>0$}

The original problem for $\mathcal{U}$ in (3.7) has nonlinearity in its principal term of the equation, which results in the complicated process in solving the linearized problem. In order to overcome this issue, we prepare a mapping which corresponds extensions of $\left(T_{0}, S_{0}\right)$ into the region $t>0$ [32].

Assume that an arbitrary $T_{1}>0$ and a pair of functions $\left(l_{2}^{\prime}, l_{3}^{\prime}\right) \in\left(W_{2}^{1+l, \frac{1+l}{2}}\left(\Omega_{T_{1}}\right)\right)^{2}$ are provided. Then, we introduce a mapping $\mathcal{V}_{1}:\left(l_{2}^{\prime}, l_{3}^{\prime}, \mathcal{U}_{0}\right) \longmapsto\left(\bar{T}_{0}, \bar{S}_{0}\right)=\left.\left(\breve{T}_{0}, \breve{S}_{0}\right)\right|_{\Omega}$, which corresponds the restriction onto $\Omega$ of a pair of solutions $\left(\breve{T}_{0}, \breve{S}_{0}\right)$ of the Cauchy problems

$$
\left\{\begin{array}{l}
\frac{\partial \breve{T}_{0}}{\partial t}-\mathcal{L}_{2,0} \breve{T}_{0}=\bar{T}_{0}^{(1)} \quad \text { in } \mathbf{R}_{T_{1}}^{3}, \quad\left\{\left.\begin{array}{l}
\frac{\partial \breve{S}_{0}}{\partial t}-\mathcal{L}_{3,0} \breve{S}_{0}=\bar{S}_{0}^{(1)} \quad \text { in } \mathbf{R}_{T_{1}}^{3}, \\
\left.\breve{T}_{0}\right|_{t=0}=T_{0} \quad \text { on } \mathbf{R}^{3},
\end{array} \breve{S}_{0}\right|_{t=0}=S_{0} \quad \text { on } \mathbf{R}^{3}\right.
\end{array}\right.
$$

to $\left(l_{2}^{\prime}, l_{3}^{\prime}, \mathcal{U}_{0}\right)$, where $\bar{T}_{0}^{(1)}$ and $\bar{S}_{0}^{(1)}$ are solutions to the following problems, respectively:

$$
\left\{\begin{array} { l } 
{ \frac { \partial \overline { T } _ { 0 } ^ { ( 1 ) } } { \partial t } - \mathcal { L } _ { 2 , 0 } \overline { T } _ { 0 } ^ { ( 1 ) } = 0 \quad \text { in } \mathbf { R } _ { T _ { 1 } } ^ { 3 } , } \\
{ \overline { T } _ { 0 } ^ { ( 1 ) } | _ { t = 0 } = l _ { 2 } ^ { \prime } \quad \text { on } \mathbf { R } ^ { 3 } , }
\end{array} \quad \left\{\begin{array}{l}
\frac{\partial \bar{S}_{0}^{(1)}}{\partial t}-\mathcal{L}_{3,0} \bar{S}_{0}^{(1)}=0 \quad \text { in } \mathbf{R}_{T_{1}}^{3}, \\
\left.\bar{S}_{0}^{(1)}\right|_{t=0}=l_{3}^{\prime} \quad \text { on } \mathbf{R}^{3} .
\end{array}\right.\right.
$$

It is obvious that $\mathcal{V}_{1}\left(l_{2}^{\prime}, l_{3}^{\prime} ; \mathcal{U}_{0}\right)=\left(\bar{T}_{0}, \bar{S}_{0}\right)$ defined above satisfies

$$
\left.\mathcal{V}_{1}\left(l_{2}^{\prime}, l_{3}^{\prime} ; \mathcal{U}_{0}\right)\right|_{t=0}=\left(T_{0}, S_{0}\right),\left.\quad \frac{\partial}{\partial t} \mathcal{V}_{1}\left(l_{2}^{\prime}, l_{3}^{\prime} ; \mathcal{U}_{0}\right)\right|_{t=0}=\left(\mathcal{L}_{2,0} T_{0}, \mathcal{L}_{3,0} S_{0}\right)+\left.\left(l_{2}^{\prime}, l_{3}^{\prime}\right)\right|_{t=0}
$$

Now, using a notation

$$
\mathcal{W}_{\mathcal{M}}\left(T_{1}\right) \equiv\left(\bar{W}_{2}^{3+l, \frac{3+l}{2}}\left(\Omega_{T_{1}}\right)\right)^{2}
$$

we advocate the following statement.

Lemma 5.1. For arbitrary $T_{1}>0,\left(l_{2}^{\prime}, l_{3}^{\prime}\right) \in\left(W_{2}^{1+l, \frac{1+l}{2}}\left(\Omega_{T_{1}}\right)\right)^{2}$ and $\mathcal{U}_{0}=\left(\mathbf{v}_{0}, T_{0}, S_{0}\right)^{\mathrm{T}} \in \mathcal{W}_{0}$, the following inequality holds:

$$
\left\|\mathcal{V}_{1}\left(l_{2}^{\prime}, l_{3}^{\prime} ; \mathcal{U}_{0}\right)\right\|_{\mathcal{W}_{\mathcal{M}}\left(T_{1}\right)} \leq C\left(\sum_{i=2}^{3}\left\|l_{i}^{\prime}\right\|_{W_{2}^{1+l, \frac{1+l}{2}}\left(\Omega_{T_{1}}\right)}+\left\|\mathcal{U}_{0}\right\|_{\mathcal{W}_{0}}\right)
$$

The proof of the lemma above is based on the classical theory of linear partial differential equations, and we omit it here.

\subsection{Problem for $\mathcal{U}$}

In this subsection, we consider linear problems for $\mathcal{U}$. For arbitrary $T_{1}>0$, let a set of functions $(\mathbf{w}, \sigma, s, f)$ be provided such that $(\mathbf{w}, \sigma, s, f) \in \bar{W}_{2, c}^{3+l, \frac{3+l}{2}}\left(\Omega_{T_{1}}\right) \times \bar{W}_{2}^{3+l, \frac{3+l}{2}}\left(\Omega_{T_{1}}\right) \times \bar{W}_{2}^{3+l, \frac{3+l}{2}}\left(\Omega_{T_{1}}\right) \times W_{2}^{\frac{5}{2}+l, \frac{5}{4}+\frac{l}{2}}\left(\mathbf{R}_{T_{1}}^{2}\right)$, $\left.(\mathbf{w}, \sigma, s, f)\right|_{t=0}=\left(\mathbf{v}_{0}, T_{0}, S_{0}, F_{0}\right)^{\mathrm{T}}$. It is also assumed the function $\tilde{p}_{*}$ satisfies

$$
\frac{\partial \tilde{p}_{*}}{\partial y_{3}}=-a^{33}(f) \varrho\left(\tilde{p}_{*}, \sigma, s\right) g \quad \text { in } \Omega_{T_{1}},\left.\quad \tilde{p}_{*}\right|_{y_{3}=F_{0}\left(y^{\prime}, t\right)}=p_{0}\left(y^{\prime}, t\right) \quad \text { on } \Gamma_{T_{1}},
$$


respectively. Define the notations $\breve{\mathcal{U}}=(\mathbf{w}, \sigma, s)$ and $\tilde{\varrho}_{*} \equiv \varrho\left(\tilde{p}_{*}, \sigma, s\right)$, and we consider the following problem with $\mathbf{l}_{i} \in \mathcal{W}_{i}\left(T_{1}\right)(i=1,2,3)$ provided:

$$
\left\{\begin{array}{l}
\frac{\partial \mathcal{U}}{\partial t}-\mathcal{L}_{\breve{\mathcal{U}}, f} \mathcal{U}=\mathbf{l}_{1} \quad \text { in } \Omega_{T_{1}} \\
\mathcal{B}_{1}\left(\nabla_{f, 3} \mathbf{w}, \tilde{\varrho}_{*}, \nabla_{f, 3} \tilde{\varrho}_{*}, f\right) \mathcal{U}=\mathbf{l}_{2} \quad \text { on } \Gamma_{T_{1}} \\
\mathcal{B}_{2}\left(\nabla_{f, 3} \mathbf{w}, \tilde{\varrho}_{*}, \nabla_{f, 3} \tilde{\varrho}_{*}, f\right) \mathcal{U}=\mathbf{l}_{3} \quad \text { on } \Gamma_{b T_{1}} \\
\left.\mathcal{U}\right|_{t=0}=\left(\mathbf{0}, T_{0}, S_{0}\right)^{\mathrm{T}} \quad \text { on } \Omega
\end{array}\right.
$$

together with the compatibility conditions up to the order 1 :

$$
\begin{cases}\left.\mathcal{B}_{1} \mathcal{U}\right|_{t=0}=\left.\mathbf{l}_{2}\right|_{t=0},\left.\frac{\partial}{\partial t} \mathcal{B}_{1} \mathcal{U}\right|_{t=0}=\left.\frac{\partial}{\partial t} \mathbf{l}_{2}\right|_{t=0} & \text { on } \Gamma \\ \left.\mathcal{B}_{2} \mathcal{U}\right|_{t=0}=\left.\mathbf{l}_{3}\right|_{t=0},\left.\frac{\partial}{\partial t} \mathcal{B}_{2} \mathcal{U}\right|_{t=0}=\left.\frac{\partial}{\partial t} \mathbf{l}_{3}\right|_{t=0} & \text { on } \Gamma_{b}\end{cases}
$$

where we have used notations defined right below (3.7):

$$
\begin{aligned}
& \mathcal{L}_{\breve{\mathcal{U}}, f} \mathcal{U} \equiv\left(L_{1, \breve{\mathcal{U}}, f} \mathbf{u}^{\prime}, L_{2, \breve{\mathcal{U}}, f} \tilde{T}, L_{3, \breve{\mathcal{U}}, f} \tilde{S}\right)^{\mathrm{T}}, \\
& \mathcal{B}_{1}\left(\nabla_{f, 3} \mathbf{w}, \tilde{\varrho}_{*}, \nabla_{f, 3} \tilde{\varrho}_{*}, f\right) \mathcal{U} \\
& \quad \equiv\left(\mathcal{B}_{11}\left(\nabla_{f, 3} \mathbf{w}, \tilde{\varrho}_{*}, \nabla_{f, 3} \tilde{\varrho}_{*}, f\right) \mathbf{u}^{\prime}, \mathcal{B}_{12}\left(\nabla_{f, 3} \mathbf{w}, \tilde{\varrho}_{*}, \nabla_{f, 3} \tilde{\varrho}_{*}, f\right) \mathcal{M}, \mathcal{B}_{13}\left(\nabla_{f, 3} \mathbf{w}, \tilde{\varrho}_{*}, \nabla_{f, 3} \tilde{\varrho}_{*}, f\right) \mathcal{M}\right)^{\mathrm{T}}, \\
& \quad \mathcal{B}_{2}\left(\nabla_{f, 3} \mathbf{w}, \tilde{\varrho}_{*}, \nabla_{f, 3} \tilde{\varrho}_{*}, f\right) \mathcal{U} \\
& \quad \equiv\left(\mathcal{B}_{21}\left(\nabla_{f, 3} \mathbf{w}, \tilde{\varrho}_{*}, \nabla_{f, 3} \tilde{\varrho}_{*}, f\right) \mathbf{u}^{\prime}, \mathcal{B}_{22}\left(\nabla_{f, 3} \mathbf{w}, \tilde{\varrho}_{*}, \nabla_{f, 3} \tilde{\varrho}_{*}, f\right) \mathcal{M}, \mathcal{B}_{23}\left(\nabla_{f, 3} \mathbf{w}, \tilde{\varrho}_{*}, \nabla_{f, 3} \tilde{\varrho}_{*}, f\right) \mathcal{M}\right)^{\mathrm{T}} .
\end{aligned}
$$

Since the principal terms of left-hand side in (5.1) includes $(\mathbf{w}, \sigma, s)$, we apply a little more complicated arguments to show the solvability of it than those with constant coefficients. The difficulties arise from the presence of the Richardon number in the principal term which includes $\frac{\partial \mathbf{v}_{0}}{\partial x_{3}}$.

In the following, we show the process of solving (5.1). We first rewrite (5.1) as follows:

$$
\left\{\begin{array}{l}
\frac{\partial \mathcal{U}}{\partial t}-\mathcal{L}_{0} \mathcal{U}=\left[\mathcal{L}_{\breve{\mathcal{U}}, f}-\mathcal{L}_{0}\right] \mathcal{U}+\mathbf{l}_{1} \equiv \mathbf{m}_{1}(\mathcal{U}) \quad \text { in } \Omega_{T_{1}}, \\
\mathcal{B}_{1,0} \mathcal{U}=\left[\mathcal{B}_{1,0}-\mathcal{B}_{1}\left(\nabla_{f, 3} \mathbf{w}, \tilde{\varrho}_{*}, \nabla_{f, 3} \tilde{\varrho}_{*}, f\right)\right] \mathcal{U}+\mathbf{l}_{2} \equiv \mathbf{m}_{2}(\mathcal{U}) \quad \text { on } \Gamma_{T_{1}}, \\
\mathcal{B}_{2,0} \mathcal{U}=\left[\mathcal{B}_{2,0}-\mathcal{B}_{2}\left(\nabla_{f, 3} \mathbf{w}, \tilde{\varrho}_{*}, \nabla_{f, 3} \tilde{\varrho}_{*}, f\right)\right] \mathcal{U}+\mathbf{l}_{3} \equiv \mathbf{m}_{3}(\mathcal{U}) \quad \text { on } \Gamma_{b T_{1}}, \\
\left.\mathcal{U}\right|_{t=0}=\left(\mathbf{0}, T_{0}, S_{0}\right)^{\mathrm{T}} \quad \text { on } \Omega
\end{array}\right.
$$

where

$$
\mathcal{L}_{0} \mathcal{U}=\left(\mathcal{L}_{1,0} \mathbf{u}^{\prime}, \mathcal{L}_{2,0} \tilde{T}, \mathcal{L}_{3,0} \tilde{S}\right)^{\mathrm{T}}, \quad \mathcal{B}_{i, 0}=\mathcal{B}_{i}\left(\frac{\partial \mathbf{v}_{0}}{\partial y_{3}}, \varrho_{u}, \frac{\partial \varrho_{u}}{\partial y_{3}}, F_{0}\right)(i=1,2),
$$

and $\varrho_{u}=\varrho\left(p_{u}, T_{0}, S_{0}\right)$ with $p_{u}(y, t)$ which is provided in (3.10). Prior to solving (5.3), we consider a linear problem with $\left(\mathbf{m}_{1}(\mathcal{U}), \mathbf{m}_{2}(\mathcal{U}), \mathbf{m}_{3}(\mathcal{U})\right)$ in $(5.3)$ replaced by the provided data $\mathbf{m}^{\prime}=\left(\mathbf{m}_{1}^{\prime}, \mathbf{m}_{2}^{\prime}, \mathbf{m}_{3}^{\prime}\right)$ satisfying 


$$
\left.\left(\mathbf{m}_{1}^{\prime}, \mathbf{m}_{2}^{\prime}, \mathbf{m}_{3}^{\prime}\right)\right|_{t=0}=\left.\left(\mathbf{l}_{1}, \mathbf{l}_{2}, \mathbf{l}_{3}\right)\right|_{t=0} .
$$

$$
\left\{\begin{array}{l}
\frac{\partial \mathcal{U}}{\partial t}-\mathcal{L}_{0} \mathcal{U}=\mathbf{m}_{1}^{\prime} \quad \text { in } \Omega_{T_{1}}, \\
\mathcal{B}_{1,0} \mathcal{U}=\mathbf{m}_{2}^{\prime} \quad \text { on } \Gamma_{T_{1}}, \\
\mathcal{B}_{2,0} \mathcal{U}=\mathbf{m}_{3}^{\prime} \quad \text { on } \Gamma_{b T_{1}}, \\
\left.\mathcal{U}\right|_{t=0}=\left(\mathbf{0}, T_{0}, S_{0}\right)^{\mathrm{T}} \quad \text { on } \Omega .
\end{array}\right.
$$

Due to (5.3), following compatibility conditions hold in this case:

$$
\begin{cases}\left.\mathcal{B}_{1,0} \mathcal{U}\right|_{t=0}=\left.\mathbf{m}_{2}^{\prime}\right|_{t=0},\left.\quad \frac{\partial}{\partial t} \mathcal{B}_{1,0} \mathcal{U}\right|_{t=0}=\left.\frac{\partial}{\partial t} \mathbf{m}_{2}^{\prime}\right|_{t=0} & \text { on } \Gamma \\ \left.\mathcal{B}_{2,0} \mathcal{U}\right|_{t=0}=\left.\mathbf{m}_{3}^{\prime}\right|_{t=0}, & \left.\frac{\partial}{\partial t} \mathcal{B}_{2,0} \mathcal{U}\right|_{t=0}=\left.\frac{\partial}{\partial t} \mathbf{m}_{3}^{\prime}\right|_{t=0} \quad \text { on } \Gamma_{b} .\end{cases}
$$

Then we apply the extension mapping defined in the previous subsection:

$$
\left(\bar{T}_{0}, \bar{S}_{0}\right)=\mathcal{V}_{1}\left(m_{12}^{\prime}, m_{13}^{\prime} ; \mathcal{U}_{0}\right)
$$

where $m_{1 i}^{\prime}(i=2,3)$ are the second and third components of $\mathbf{m}_{1}^{\prime}$. Introducing notations $\widetilde{\mathcal{U}}=\left(\mathbf{u}^{\prime}, T-\bar{T}_{0}, S-\bar{S}_{0}\right)^{\mathrm{T}}$ and $\mathcal{U}_{0}^{\prime}=\left(\mathbf{0}, \bar{T}_{0}, \bar{S}_{0}\right)^{\mathrm{T}}$, we have from $(5.4)$

$$
\left\{\begin{array}{l}
\frac{\partial \widetilde{\mathcal{U}}}{\partial t}-\mathcal{L}_{0} \tilde{\mathcal{U}}=\mathbf{m}_{1}^{\prime}-\left(\frac{\partial}{\partial t}-\mathcal{L}_{0}\right) \mathcal{U}_{0}^{\prime} \equiv \mathbf{k}_{1} \quad \text { in } \Omega_{T} \\
\mathcal{B}_{1,0} \widetilde{\mathcal{U}}=\mathbf{m}_{2}^{\prime}-\mathcal{B}_{1,0} \mathcal{U}_{0}^{\prime} \equiv \mathbf{k}_{2} \quad \text { on } \Gamma_{T}, \\
\mathcal{B}_{2,0} \widetilde{\mathcal{U}}=\mathbf{m}_{3}^{\prime}-\mathcal{B}_{2,0} \mathcal{U}_{0}^{\prime} \equiv \mathbf{k}_{3} \quad \text { on } \Gamma_{b T}, \\
\left.\widetilde{\mathcal{U}}\right|_{t=0}=\mathbf{0} \quad \text { on } \Omega .
\end{array}\right.
$$

It is easily seen that the right-hand sides of (5.6) satisfies $\left.\mathbf{k}_{i}\right|_{t=0}=0(i=1,2,3),\left.\frac{\partial}{\partial t} \mathbf{k}_{i}\right|_{t=0}=\mathbf{0} \quad(i=2,3)$, and $\left(\mathbf{k}_{1}, \mathbf{k}_{2}, \mathbf{k}_{3}\right) \in \mathcal{W}_{\mathcal{H}}\left(T_{1}\right) \equiv \prod_{i=1}^{3} \mathcal{W}_{i}\left(T_{1}\right)$. In addition, the regularity of coefficients in operators in the left-hand side of (5.6) are sufficient in the sense that we are able to apply the regularizer method. Accordingly, by virtue of the classical result of the linear partial differential equation, we have the following result.

Theorem 5.2. Assume $F_{0} \in W_{2}^{\frac{5}{2}+l}\left(\mathbf{R}^{2}\right), \mathcal{U}_{0}=\left(\mathbf{v}_{0}, T_{0}, S_{0}\right)^{\mathrm{T}} \in \mathcal{W}_{0},\left(1+\alpha_{i} \mathcal{R}_{0}\right)\left|\frac{\partial \mathbf{v}_{0}}{\partial x_{3}}\right| \geq c_{u}>0(i=2,4,6)$. Let $T_{1}>0$ and $f$ satisfy the assumption $\left(A_{f}\right)$. In addition, the compatibility conditions up to the order 1 are assumed to hold. Then, there exists $T_{51} \in\left(0, T_{1}\right]$ and a unique solution $\widetilde{\mathcal{U}} \in \mathcal{W}_{\mathcal{U}}^{\prime}\left(T_{51}\right)$ to (5.6) satisfying

$$
\|\widetilde{\mathcal{U}}\|_{\mathcal{W}_{\mathcal{U}}^{\prime}\left(T_{51}\right)} \leq C \sum_{i=1}^{3}\left\|\mathbf{k}_{i}\right\|_{\mathcal{W}_{i}\left(T_{51}\right)} .
$$

The proof of this theorem is substantial to the main result of this paper, but is somewhat lengthy, and will be provided in the next subsection. This theorem obviously implies that the problem (5.4) has a unique solution $\mathcal{U}$ satisfying

$$
\|\mathcal{U}\|_{\mathcal{W}_{\mathcal{U}}\left(T_{51}\right)} \leq C\left\|\mathbf{m}^{\prime}\right\|_{\mathcal{W}_{\mathcal{H}}\left(T_{51}\right)},
$$

where the function space $\mathcal{W}_{\mathcal{U}}\left(T_{51}\right)$ is defined in the statement of Lemma 4.10. Next, we show the unique existence of the solution to the problem (5.2). 
Theorem 5.3. Assume $F_{0} \in W_{2}^{\frac{5}{2}+l}\left(\mathbf{R}^{2}\right), \mathcal{U}_{0} \equiv\left(\mathbf{v}_{0}, T_{0}, S_{0}\right)^{\mathrm{T}} \in \mathcal{W}_{0}, \min \left\{\left(1+\alpha_{i} \mathcal{R}_{0}\right)\left|\frac{\partial \mathbf{v}_{0}}{\partial y_{3}}\right|,\left|\frac{\partial \mathbf{v}_{0}}{\partial y_{3}}\right|\right\} \geq c_{u}>$ $0(i=2,4,6)$. Define $\mathcal{R}_{*}$ as that in Lemma 4.10 with $\left(\mathbf{u}_{*}, \tilde{T}_{*}, \tilde{S}_{*}, F_{*}\right)$ replaced by $(\mathbf{w}, \sigma, s, f)$, and $\mathbf{w}^{\prime}=\mathbf{w}-\tilde{\mathbf{\mathbf { v }}}_{0}^{(f)}$. Let $T_{1}>0$ and $f$ satisfy the assumption $\left(A_{f}\right), \mathbf{l}_{i} \in \mathcal{W}_{i}\left(T_{1}\right)(i=1,2,3)$, and assume in addition

$$
\min \left\{\left(1+\alpha_{i} \mathcal{R}_{*}\right)\left|\frac{\partial \mathbf{w}}{\partial y_{3}}\right|,\left|\frac{\partial \mathbf{w}}{\partial y_{3}}\right|\right\} \geq c_{u}>0
$$

holds for $t \in\left(0, T_{1}\right]$. We also assume that the compatibility conditions (5.2) hold. Then, under the condition

$$
\begin{gathered}
C_{0}\left(T_{1}\right)\left\{\left\|\mathbf{w}^{\prime}\right\|_{W_{2}^{3+l, \frac{3+l}{2}}\left(\Omega_{T_{1}}\right)}+\sum_{1 \leq 2 \chi_{1}+\left|\chi_{2}\right| \leq 3}\left[\left\|D_{t}^{\chi_{1}} D_{y}^{\chi_{2}} \sigma\right\|_{W_{2}^{l, \frac{l}{2}}\left(\Omega_{T_{1}}\right)}+\left\|D_{t}^{\chi_{1}} D_{y}^{\chi_{2}} s\right\|_{W_{2}^{l, \frac{l}{2}}\left(\Omega_{T_{1}}\right)}\right]\right. \\
\left.+\left\|f-F_{0}\right\|_{W_{2}^{\frac{5}{2}+l, \frac{5}{4}+\frac{l}{2}}\left(\mathbf{R}_{T_{1}}^{2}\right)}\right\}<\delta
\end{gathered}
$$

with some power function $C_{0}(\cdot)$ and a positive constant $\delta$, there exists $T_{52} \in\left(0, T_{1}\right]$ and a unique solution $\mathcal{U} \in \mathcal{W}_{\mathcal{U}}\left(T_{52}\right)$ to $(5.1)$ satisfying

$$
\|\mathcal{U}\|_{\mathcal{W}_{\mathcal{U}}\left(T_{52}\right)} \leq C \sum_{i=1}^{3}\left\|\mathbf{l}_{i}\right\|_{\mathcal{W}_{i}\left(T_{52}\right)} .
$$

Proof. Define a mapping $\mathcal{V}_{2}$ that corresponds the solution $\mathcal{U}$ to $\left(\mathbf{m}_{1}, \mathbf{m}_{2}, \mathbf{m}_{3}\right)$ in (5.3), which is assured to exist by Theorem 5.2. Then, the problem (5.1) can be interpreted as the following functional equation:

$$
\mathcal{U}=\mathcal{V}_{2}\left(\left(\mathbf{m}_{1}, \mathbf{m}_{2}, \mathbf{m}_{3}\right)(\mathcal{U})\right) .
$$

The estimate (4.12) in Lemma 4.10 indicates that the right-hand side of above equality is a contraction map on $\mathcal{W}_{\mathcal{U}}\left(T_{52}\right)$ over a short time interval $\left(0, T_{52}\right]$, which is obviously continuous at the same time. Combining the estimates stated in Lemmas 4.10 and 5.1, we have the desired estimate.

\subsection{Proof of Theorem 5.2}

Now we show the proof of Theorem 5.2. The basic idea of the proof of Theorem 5.2 is the combination of the abstract argument of the functional analysis and the regularizer method [32]. Let $\mathcal{A}$ be a linear operator defined in the space $\mathcal{W}_{\mathcal{U}}^{\prime}\left(T_{1}\right)$ which corresponds $\widetilde{\mathcal{U}} \in \mathcal{W}_{\mathcal{U}}^{\prime}\left(T_{1}\right)$ to $\left(\mathcal{L}_{0} \widetilde{\mathcal{U}},\left.\mathcal{B}_{1,0} \widetilde{\mathcal{U}}\right|_{\Gamma},\left.\mathcal{B}_{2,0} \widetilde{\mathcal{U}}\right|_{\Gamma_{b}}\right)$. By virtue of $(4.11)$ in Lemma 4.10, it is obvious that the operator $\mathcal{A}$ is bounded. Then problem (5.6) is interpreted as the problem of solving the abstract equation

$$
\mathcal{A} \tilde{\mathcal{U}}=\mathcal{H}
$$

for $\mathcal{H} \in \mathcal{W}_{\mathcal{H}}\left(T_{1}\right)=\prod_{i=1}^{3} \mathcal{W}_{i}\left(T_{1}\right)$ (see the definition right above Lem. 4.9), and what we have to do is to show the existence of a bounded inverse operator $\mathcal{A}^{-1}$. In order to prove Theorem 5.2, we will construct an operator $\mathcal{R}$ acting from $\mathcal{W}_{\mathcal{H}}\left(T_{1}\right)$ into $\mathcal{W}_{\mathcal{U}}^{\prime}\left(T_{1}\right)$ and such that for any $\mathcal{H} \in \mathcal{W}_{\mathcal{H}}\left(T_{1}\right)$ and $\widetilde{\mathcal{U}} \in \mathcal{W}_{\mathcal{U}}^{\prime}\left(T_{1}\right)$,

$$
\begin{aligned}
& \mathcal{A} \mathcal{R} \mathcal{H}=\mathcal{H}+\mathcal{T H}, \\
& \mathcal{R} \mathcal{A} \widetilde{\mathcal{U}}=\widetilde{\mathcal{U}}+W \widetilde{\mathcal{U}},
\end{aligned}
$$

where $\mathcal{T}$ and $W$ are bounded operators in the spaces $\mathcal{W}_{\mathcal{H}}\left(T_{1}\right)$ and $\mathcal{W}_{\mathcal{U}}^{\prime}\left(T_{1}\right)$, respectively, whose norms are small if $T_{1}$ is sufficiently small. The overview of the line of the proof is as follows:

- We introduce coverings of the region $\Omega$;

- In each piece of the covering, we consider the model problem in the half-space in a local chart; 
- We construct the operators $\mathcal{T}$ and $W$ above, and show the smallness of their norms under small $T_{1}$;

- We show the existence of $\mathcal{A}^{-1}$ under the small $T_{1}$, and extend the time interval by the continuation argument.

In the following, we denote the original coordinate system in $\Omega$ by $y$ to avoid confusion.

\subsubsection{Coverings of $\Omega$}

We first introduce coverings of $\Omega$, on which we construct the regularizer later [32]. Take an arbitrary point $\xi \in \Gamma$, around which $\Gamma$ is represented by the equation $\tilde{y}_{3}=F_{\xi}\left(\tilde{y}^{\prime}\right)$. Here $\tilde{y}=\left(\tilde{y}^{\prime}, \tilde{y}_{3}\right)=\left(\tilde{y}_{1}, \tilde{y}_{2}, \tilde{y}_{3}\right)$ is a local coordinate system with the origin at $\xi$ and with $\tilde{y}_{3}$-axis directed along $\mathbf{N}_{F_{0}}$ and $F_{\xi} \in W_{2}^{\frac{5}{2}+l}\left(\mathbf{R}^{2}\right)$. The same situation holds on $\Gamma_{b}$, that is, around an arbitrary point $\xi \in \Gamma_{b}, \Gamma_{b}$ is represented by the equation $\tilde{y}_{3}=b_{\xi}\left(\tilde{y}^{\prime}\right)$ with the local chart $\tilde{y}$, whose origin is located at $\xi$. We also note that $F_{\xi}$ is defined on a circle $K_{r} \equiv\left\{\tilde{y}^{\prime} \in \mathbf{R}^{2} \| \tilde{y}^{\prime} \mid<r\right\}$ and satisfies $F_{\xi}(0)=\nabla F_{\xi}(0)=0$, and $\left\|F_{\xi}\right\|_{W_{2}^{\frac{5}{2}+l}\left(\mathbf{R}^{2}\right)} \leq M_{1}$. This holds for $\Gamma_{b}$ also by replacing $F_{\xi}$ by $b_{\xi}$. Then we introduce two systems of of covering of $\Omega$, say, $\left\{\omega^{(k)}\right\}$ and $\left\{\Omega^{(k)}\right\}$, which are constructed for any small number $\lambda$, satisfying $0<\lambda<\min \left\{r, c_{0} / 2\right\}$ as follows ( $c_{0}$ was introduced in the statement of Thm. 3.1):

(i) $\omega^{(k)} \subset \Omega^{(k)} \subset \Omega \quad \forall k, \bigcup_{k} \omega^{(k)}=\bigcup_{k} \Omega^{(k)}=\Omega$;

(ii) for any $\xi \in \Omega$, there exists $\omega^{(k)}$ such that $y \in \omega^{(k)}$ and $\operatorname{dist}\left(y, \Omega \backslash \omega^{(k)}\right) \geq \beta_{1} \lambda$ with some $\beta_{1}$;

(iii) for any $\lambda>0$, there exists a natural number $N_{0}$ independent of $\lambda$ such that $\bigcap_{k=1}^{N_{0}+1} \Omega^{(k)}$ is a null set;

(iv) if $\Omega^{(k)} \cap\left(\Gamma \cup \Gamma_{b}\right)$ is a null set, then $\omega^{(k)}$ and $\Omega^{(k)}$ are the cubes with the same center $\xi^{(k)}$ and with the length of their edges equal to $\frac{\lambda}{2}$ and $\lambda$, respectively. In this case, we represent $k \in \mathcal{N}_{1}$;

(v) if $\omega^{(k)} \cap \Gamma \neq \phi$, then by the local rectangular coordinate system $\{\tilde{y}\}$ with the origin at $\xi \in \Gamma$, $\omega^{(k)}$ and $\Omega^{(k)}$ are defined by the inequalities

$$
\begin{gathered}
\left|\tilde{y}_{i}\right|<\frac{\lambda}{2}(i=1,2) ; 0<\tilde{y}_{3}-F_{\xi}\left(\tilde{y}^{\prime}\right)<\frac{\lambda}{2}, \\
\left|\tilde{y}_{i}\right|<\lambda(i=1,2) ; 0<\tilde{y}_{3}-F_{\xi}\left(\tilde{y}^{\prime}\right)<\lambda .
\end{gathered}
$$

Similarly, if $\omega^{(k)} \cap \Gamma_{b} \neq \phi$, then by the local rectangular coordinate system $\{\tilde{y}\}$ with the origin at $\xi \in \Gamma_{b}$, $\omega^{(k)}$ and $\Omega^{(k)}$ are defined by the inequalities

$$
\begin{aligned}
& \left|\tilde{y}_{i}\right|<\frac{\lambda}{2}(i=1,2) ; 0<\tilde{y}_{3}-b_{\xi}\left(\tilde{y}^{\prime}\right)<\frac{\lambda}{2}, \\
& \left|\tilde{y}_{i}\right|<\lambda(i=1,2) ; 0<\tilde{y}_{3}-b_{\xi}\left(\tilde{y}^{\prime}\right)<\lambda .
\end{aligned}
$$

In case $\Omega^{(k)} \bigcap \Gamma \neq \phi$, we represent $k \in \mathcal{N}_{2}$, while in case $\Omega^{(k)} \bigcap \Gamma_{b} \neq \phi, k \in \mathcal{N}_{3}$.

Next, let us introduce a smooth partition of unity $\left\{\zeta^{(k)}(y)\right\}$ subordinated to $\left\{\omega^{(k)}\right\}$ and $\left\{\Omega^{(k)}\right\}$ having the following properties with a multi-index $\alpha$ :

$$
0 \leq \zeta^{(k)}(y) \leq 1, \quad\left|D_{y}^{\alpha} \zeta^{(k)}(y)\right| \leq \frac{c_{\alpha}}{\lambda^{|\alpha|}}, \quad \zeta^{(k)}(y)= \begin{cases}1 & \text { for } y \in \omega^{(k)}, \\ 0 & \text { for } y \in \Omega \backslash \Omega^{(k)}\end{cases}
$$

with some constant $c_{\alpha}$ independent of $k$ and $\lambda$. By virtue of the property (iii) of $\Omega^{(k)}, 1 \leq \sum_{k}\left(\zeta^{(k)}(y)\right)^{2} \leq N_{0}$, and it is possible to take the functions

$$
\eta^{(k)}(y) \equiv \frac{\zeta^{(k)}(y)}{\left(\sum_{j} \zeta^{(j)}(y)\right)^{2}} \quad k \in \bigcup_{i=1}^{3} \mathcal{N}_{i}
$$


having the properties

$$
\eta^{(k)}(y)=0 \quad \text { in } \Omega \backslash \Omega^{(k)}, \quad\left|D_{y}^{\alpha} \eta^{(k)}(y)\right| \leq \frac{c_{\alpha}}{\lambda^{|\alpha|}}, \quad \sum_{k} \eta^{(k)}(y) \zeta^{(k)}(y)=1
$$

\subsubsection{Problems in half-space}

Now we consider the model problems in the half-space $\mathbf{R}_{(+)}^{3} \equiv \mathbf{R}^{2} \times(0, \infty)$ for each affiliation of $k$. In the following, let $T_{1}$ be an arbitrary positive number. We introduce following notations to represent the coordinate system of coefficients and the derivatives of the operators explicitly:

$$
\mathcal{L}_{0}\left(y, t, \frac{\partial}{\partial y}\right), \mathcal{B}_{i, 0}\left(y, t, \frac{\partial}{\partial y}\right) \quad(i=1,2)
$$

where $\frac{\partial}{\partial y}=\left(\frac{\partial}{\partial y_{1}}, \frac{\partial}{\partial y_{2}}, \frac{\partial}{\partial y_{3}}\right)^{\mathrm{T}}$. First, we show the case $k \in \mathcal{N}_{1}$. We consider the problem

$$
\left\{\begin{array}{l}
\frac{\partial \widetilde{\mathcal{U}}^{(k)}}{\partial t}-\mathcal{L}_{0}\left(\xi^{(k)}, 0, \frac{\partial}{\partial y}\right) \widetilde{\mathcal{U}}^{(k)}=\mathbf{k}_{1}^{(k)} \text { in } \mathbf{R}_{(+) T_{1}}^{3} \equiv \mathbf{R}_{(+)}^{3} \times\left(0, T_{1}\right), \\
\left.\widetilde{\mathcal{U}}^{(k)}\right|_{t=0}=\mathbf{0} \text { on } \mathbf{R}_{(+)}^{3},
\end{array}\right.
$$

for certain $\xi^{(k)} \in\left\{\left(y^{\prime}, 0\right) \mid y^{\prime} \in \mathbf{R}^{2}\right\}$ and $\mathbf{k}_{1}^{(k)}=\zeta^{(k)} \mathbf{k}_{1} \in \mathcal{W}_{1(+)}\left(T_{1}\right) \equiv\left(W_{2}^{1+l, \frac{1+l}{2}}\left(\mathbf{R}_{(+) T_{1}}^{3}\right)\right)^{3}$.

It is well known that the problem (5.11) has a unique solution $\widetilde{\mathcal{U}}^{(k)} \in\left(W_{2}^{3+l, \frac{3+l}{2}}\left(\mathbf{R}_{(+) T_{1}}^{3}\right)\right)^{3}[32]$, which is explicitly provided by applying the Fourier-Laplace transform and the rotation of the coordinate system. We denote by $R^{(k)}$ the operator that corresponds this solution $\mathcal{U}^{(k)}$ to $\mathbf{k}_{1}^{(k)}$ in (5.11).

Next, we consider the case $k \in \mathcal{N}_{2}$. Hereafter we use notations $\mathcal{B}_{i, 0}^{(H)}(i=1,2,3)$ representing operators consisting of the terms of derivative in $\mathcal{B}_{i, 0}(i=1,2,3)$. In addition, for $k \in \bigcup_{i=2}^{3} \mathcal{N}_{i}$, let $\mathcal{L}_{0}^{(k)}\left(\tilde{y}, t, \frac{\partial}{\partial \tilde{y}}\right), \overline{\mathcal{B}}_{i, 0}^{(H)(k)}\left(\tilde{y}, t, \frac{\partial}{\partial \tilde{y}}\right)$ be the operators $\left(\mathcal{L}_{0}\right.$ and $\mathcal{B}_{i, 0}^{(H)}(i=1,2,3)$ in the local chart $\{\tilde{y}\}$ around a point $\xi^{(k)}=\left(\xi_{1}^{(k)}, \xi_{2}^{(k)}, \xi_{3}^{(k)}\right)^{\mathrm{T}}$. Note that the coordinate systems $\{y\}$ and $\{\tilde{y}\}$ are related with a rotation and movement of the coordinate system:

$$
\tilde{y}_{i}=\sum_{j=1}^{3} \beta_{i j}^{(k)}\left(y_{j}-\xi_{j}^{(k)}\right)(i=1,2,3),
$$

where $\left[\beta_{i j}^{(k)}\right]_{i, j=1,2,3}$ is an orthogonal matrix. Now, let us introduce a notation of the coordinate transform $Z_{k}: f(z) \longmapsto \tilde{f}(y) \equiv f(z(y))$ on $\Omega^{(k)}$ from $z$-coordinate system to $y$-coordinate system, where the relationship between $z$ - and $\tilde{y}$-coordinate system is provided by

$$
z^{\prime}=\tilde{y}^{\prime}, \quad z_{3}=\left\{\begin{array}{l}
\tilde{y}_{3}-F_{\xi}\left(\tilde{y}^{\prime}\right) . \\
\tilde{y}_{3}-b_{\xi}\left(\tilde{y}^{\prime}\right),
\end{array}\right.
$$

while the one between $\tilde{y}$ - and $y$-coordinate system is provided above. Hereafter in this subsection, we use the term "z-coordinate" referring to the local chart after this transform. Note that it is different from the one used in Section 1.1 in the literature of oceanography. Then, rewriting the problem in $z$-coordinate system, we consider 
the following initial boundary value problem in the half-space:

$$
\left\{\begin{array}{l}
\frac{\partial \widetilde{\mathcal{U}}^{(k)}}{\partial t}-\mathcal{L}_{0}^{(k)}\left(\mathbf{0}, 0, \frac{\partial}{\partial z}\right) \widetilde{\mathcal{U}}^{(k)}=\mathbf{k}_{1}^{(k)} \quad \text { in } \mathbf{R}_{(+) T_{1}}^{3}, \\
\overline{\mathcal{B}}_{1,0}^{(H)(k)}\left(\mathbf{0}, 0, \frac{\partial}{\partial z}\right) \widetilde{\mathcal{U}}^{(k)}=\mathbf{k}_{2}^{(k)} \equiv \zeta^{(k)} \mathbf{k}_{2} \quad \text { on } \mathbf{R}_{T_{1}}^{2} \equiv \mathbf{R}^{2} \times\left(0, T_{1}\right) \\
\left.\widetilde{\mathcal{U}}^{(k)}\right|_{t=0}=\mathbf{0} \quad \text { on } \mathbf{R}_{(+)}^{3} .
\end{array}\right.
$$

This problem also has a unique solution $\widetilde{\mathcal{U}}^{(k)} \in\left(W_{2}^{3+l, \frac{3+l}{2}}\left(\mathbf{R}_{(+) T_{1}}^{3}\right)\right)^{3}$ by applying the Fourier-Laplace transform [32], and we denote by $R^{(k)}$ the operator that associates with $h^{(k)}=\left(\mathbf{k}_{1}^{(k)}, \mathbf{k}_{2}^{(k)}\right) \in \mathcal{W}_{1(+)}\left(T_{1}\right) \times \mathcal{W}_{2(+)}\left(T_{1}\right)$ the solution of the problem above, where

$$
\mathcal{W}_{2(+)}\left(T_{1}\right) \equiv\left(W_{2}^{\frac{3}{2}+l, \frac{3}{4}+\frac{l}{2}}\left(\mathbf{R}_{T_{1}}^{2}\right)\right)^{3} .
$$

For $k \in \mathcal{N}_{3}$, let $\mathcal{L}_{0}^{(k)}\left(\tilde{y}, t, \frac{\partial}{\partial \tilde{y}}\right), \overline{\mathcal{B}}_{2,0}^{(H)(k)}$ be the operators $\left(\mathcal{L}_{0}, \mathcal{B}_{2,0}^{(H)}\right)$ in the local chart $\{\tilde{y}\}$ at the point $\xi^{(k)} \in \Gamma_{b}$.

Then we consider the problem in the $z$-coordinate system

$$
\left\{\begin{array}{l}
\frac{\partial \widetilde{\mathcal{U}}^{(k)}}{\partial t}-\mathcal{L}_{0}^{(k)}\left(\mathbf{0}, 0, \frac{\partial}{\partial z}\right) \widetilde{\mathcal{U}}^{(k)}=\mathbf{k}_{1}^{(k)} \text { in } \mathbf{R}_{(+) T_{1}}^{3} \\
\overline{\mathcal{B}}_{2,0}^{(H)(k)}\left(\mathbf{0}, 0, \frac{\partial}{\partial z}\right) \widetilde{\mathcal{U}}^{(k)}=\mathbf{k}_{3}^{(k)} \equiv \zeta^{(k)} \mathbf{k}_{3} \quad \text { on } \mathbf{R}_{T_{1}}^{2} \\
\left.\widetilde{\mathcal{U}}^{(k)}\right|_{t=0}=\mathbf{0} \quad \text { on } \mathbf{R}_{(+)}^{3} .
\end{array}\right.
$$

This problem also has a unique solution $\widetilde{\mathcal{U}}^{(k)} \in\left(W_{2}^{3+l, \frac{3+l}{2}}\left(\mathbf{R}_{(+) T_{1}}^{3}\right)\right)^{3}$ by applying the Fourier-Laplace transform [32], and we denote by $R^{(k)}$ the operator that associates with $h^{(k)}=\left(\mathbf{k}_{1}^{(k)}, \mathbf{k}_{3}^{(k)}\right) \in \mathcal{W}_{1(+)}\left(T_{1}\right) \times \mathcal{W}_{2(+)}\left(T_{1}\right)$ the solution of the problem above. Define an operator $\mathcal{R}$, which associates $\mathcal{H}=\left(\mathbf{k}_{1}, \mathbf{k}_{2}, \mathbf{k}_{3}\right) \in \mathcal{W}_{\mathcal{H}}\left(T_{1}\right)$ the following items:

$$
\mathcal{R H}=\sum_{k} \eta^{(k)}(y) \widetilde{\mathcal{U}}^{(k)}(y, t)
$$

where

$$
\widetilde{\mathcal{U}}^{(k)}(y, t)= \begin{cases}R^{(k)} \zeta^{(k)} \mathbf{k}_{1} \quad\left(k \in \mathcal{N}_{1}\right), & \\ Z_{k} R^{(k)}\left(Z_{k}^{-1} \zeta^{(k)} \mathbf{k}_{1}, Z_{k}^{-1} \zeta^{(k)} \mathbf{k}_{2}\right) & \left(k \in \mathcal{N}_{2}\right), \\ Z_{k} R^{(k)}\left(Z_{k}^{-1} \zeta^{(k)} \mathbf{k}_{1}, Z_{k}^{-1} \zeta^{(k)} \mathbf{k}_{3}\right) & \left(k \in \mathcal{N}_{3}\right) .\end{cases}
$$

By virtue of the classical result of the half-space problem, we have the estimates

$$
\begin{aligned}
&\left\|R^{(k)} \mathbf{k}_{1}\right\|_{W_{2}^{3+l, \frac{3+l}{2}}\left(\mathbf{R}_{(+) T_{1}}^{3}\right)} \leq C\left\|\mathbf{k}_{1}\right\|_{\mathcal{W}_{1(+)}\left(T_{1}\right)} \quad \text { for } \quad k \in \mathcal{N}_{1}, \\
&\left\|R^{(k)}\left(\mathbf{k}_{1}, \mathbf{k}_{i}\right)\right\|_{W_{2}^{3+l, \frac{3+l}{2}}\left(\mathbf{R}_{(+) T_{1}}^{3}\right)} \leq C\left(\left\|\mathbf{k}_{1}\right\|_{\mathcal{W}_{1(+)}\left(T_{1}\right)}+\left\|\mathbf{k}_{i}\right\|_{\mathcal{W}_{2(+)}\left(T_{1}\right)}\right) \quad \text { for } \quad k \in \mathcal{N}_{i}(i=2,3) .
\end{aligned}
$$

From this, we have the following lemma without proof. 
Lemma 5.4. For arbitrary $T_{1}>0$, the operator $\mathcal{R}$ is a bounded operator acting from the space $\mathcal{W}_{\mathcal{H}}\left(T_{1}\right)$ into the space $\mathcal{W}_{\mathcal{U}}^{\prime}\left(T_{1}\right)$, and is subject to the estimate

$$
\|\mathcal{R} \mathcal{H}\|_{\mathcal{W}_{\mathcal{U}}^{\prime}\left(T_{1}\right)} \leq C\|\mathcal{H}\|_{\mathcal{W}_{\mathcal{H}}\left(T_{1}\right)}
$$

Now we construct operators $\mathcal{T}$ and $W$ which are shown to be bounded on the spaces $\mathcal{W}_{\mathcal{H}}\left(T_{1}\right)$ and $\mathcal{W}_{\mathcal{U}}^{\prime}\left(T_{1}\right)$, respectively. We define

$$
\begin{aligned}
& \mathcal{A}_{0} \tilde{\mathcal{U}}=\left(\mathcal{L}_{0} \tilde{\mathcal{U}},\left.\mathcal{B}_{1,0}^{(H)} \tilde{\mathcal{U}}\right|_{\Gamma},\left.\mathcal{B}_{2,0}^{(H)} \tilde{\mathcal{U}}\right|_{\Gamma_{b}}\right)^{\mathrm{T}} \\
& \mathcal{A}_{1} \tilde{\mathcal{U}}=\left(\mathcal{L}_{0}^{\prime} \tilde{\mathcal{U}},\left.\mathcal{B}_{1,0}^{(L)} \widetilde{\mathcal{U}}\right|_{\Gamma},\left.\mathcal{B}_{2,0}^{(L)} \tilde{\mathcal{U}}\right|_{\Gamma_{b}}\right)^{\mathrm{T}}
\end{aligned}
$$

where $\mathcal{B}_{i, 0}^{(L)}=\mathcal{B}_{i, 0}-\mathcal{B}_{i, 0}^{(H)}(i=1,2)$. By noting

$$
\begin{aligned}
& \left(\frac{\partial}{\partial t}-\mathcal{L}_{0}\left(\xi^{(k)}, 0, \frac{\partial}{\partial y}\right)\right) \widetilde{\mathcal{U}}^{(k)} \\
& =\left\{\begin{array}{r}
\zeta^{(k)} \mathbf{k}_{1} \quad\left(k \in \mathcal{N}_{1}\right), \\
Z_{k}\left[\mathcal{L}_{0}^{(k)}\left(\mathbf{0}, 0, \frac{\partial}{\partial z}-\nabla_{3} F_{\xi^{(k)}} \frac{\partial}{\partial z_{3}}\right)-\mathcal{L}_{0}^{(k)}\left(\mathbf{0}, 0, \frac{\partial}{\partial z}\right)\right] R^{(k)}\left(\begin{array}{r}
\left.Z_{k}^{-1} \zeta^{(k)} \mathbf{k}_{1}, Z_{k}^{-1} \zeta^{(k)} \mathbf{k}_{2}\right) \\
+\zeta^{(k)} \mathbf{k}_{1} \quad
\end{array} \quad\left(k \in \mathcal{N}_{2}\right),\right. \\
Z_{k}\left[\mathcal{L}_{0}^{(k)}\left(\mathbf{0}, 0, \frac{\partial}{\partial z}-\nabla_{3} b_{\xi^{(k)}} \frac{\partial}{\partial z_{3}}\right)-\mathcal{L}_{0}^{(k)}\left(\mathbf{0}, 0, \frac{\partial}{\partial z}\right)\right] R^{(k)}\left(\begin{array}{r}
\left.Z_{k}^{-1} \zeta^{(k)} \mathbf{k}_{1}, Z_{k}^{-1} \zeta^{(k)} \mathbf{k}_{3}\right) \\
+\zeta^{(k)} \mathbf{k}_{1}
\end{array} \quad\left(k \in \mathcal{N}_{3}\right),\right.
\end{array}\right.
\end{aligned}
$$

we have $\left(\nabla_{3}\right.$ is defined right after (1.5))

$$
\begin{aligned}
\left(\frac{\partial}{\partial t}-\mathcal{L}_{0}\right) \mathcal{R H}= & \sum_{k}\left[\left(\frac{\partial}{\partial t}-\mathcal{L}_{0}\right) \eta^{(k)} \widetilde{\mathcal{U}}^{(k)}\right] \\
= & \sum_{k} \eta^{(k)} \frac{\partial \widetilde{\mathcal{U}}^{(k)}}{\partial t}-\sum_{k}\left(\mathcal{L}_{0} \eta^{(k)} \widetilde{\mathcal{U}}^{(k)}-\eta^{(k)} \mathcal{L}_{0} \widetilde{\mathcal{U}}^{(k)}\right) \\
& -\sum_{k} \eta^{(k)}\left[\mathcal{L}_{0}\left(y, t, \frac{\partial}{\partial y}\right)-\mathcal{L}_{0}\left(\xi^{(k)}, 0, \frac{\partial}{\partial y}\right)\right] \widetilde{\mathcal{U}}^{(k)}-\sum_{k} \eta^{(k)} \mathcal{L}_{0}\left(\xi^{(k)}, 0, \frac{\partial}{\partial y}\right) \widetilde{\mathcal{U}}^{(k)} \\
= & \mathbf{k}_{1}+\mathcal{T}_{1} \mathcal{H},
\end{aligned}
$$

where

$$
\begin{aligned}
\mathcal{T}_{1} \mathcal{H} \equiv & -\sum_{k}\left(\mathcal{L}_{0} \eta^{(k)} \widetilde{\mathcal{U}}^{(k)}-\eta^{(k)} \mathcal{L}_{0} \widetilde{\mathcal{U}}^{(k)}\right)-\sum_{k} \eta^{(k)}\left[\mathcal{L}_{0}\left(y, t, \frac{\partial}{\partial y}\right)-\mathcal{L}_{0}\left(\xi^{(k)}, 0, \frac{\partial}{\partial y}\right)\right] \widetilde{\mathcal{U}}^{(k)} \\
& -\sum_{k \in \mathcal{N}_{2}} \eta^{(k)} Z_{k}\left[\mathcal{L}_{0}^{(k)}\left(\mathbf{0}, 0, \frac{\partial}{\partial z}-\nabla_{3} F_{\xi^{(k)}} \frac{\partial}{\partial z_{3}}\right)-\mathcal{L}_{0}^{(k)}\left(\mathbf{0}, 0, \frac{\partial}{\partial z}\right)\right] R^{(k)}\left(Z_{k}^{-1} \zeta^{(k)} \mathbf{k}_{1}, Z_{k}^{-1} \zeta^{(k)} \mathbf{k}_{2}\right) \\
& -\sum_{k \in \mathcal{N}_{3}} \eta^{(k)} Z_{k}\left[\mathcal{L}_{0}^{(k)}\left(\mathbf{0}, 0, \frac{\partial}{\partial z}-\nabla_{3} b_{\xi^{(k)}} \frac{\partial}{\partial z_{3}}\right)-\mathcal{L}_{0}^{(k)}\left(\mathbf{0}, 0, \frac{\partial}{\partial z}\right)\right] R^{(k)}\left(Z_{k}^{-1} \zeta^{(k)} \mathbf{k}_{1}, Z_{k}^{-1} \zeta^{(k)} \mathbf{k}_{3}\right) .
\end{aligned}
$$


In the analogous way, we have

$$
\mathcal{B}_{i, 0} \mathcal{R U}=\mathbf{k}_{i+1}+\mathcal{T}_{i+1} \mathcal{H} \quad(i=1,2)
$$

where

$$
\begin{aligned}
\mathcal{T}_{2} \mathcal{H} \equiv & \left.\overline{\mathcal{B}}_{1,0}^{(L)} \mathcal{R} \mathcal{H}\right|_{\Gamma}+\left.\sum_{k \in \mathcal{N}_{2}}\left(\overline{\mathcal{B}}_{1,0}^{(H)} \eta^{(k)} \widetilde{\mathcal{U}}^{(k)}-\eta^{(k)} \overline{\mathcal{B}}_{1,0}^{(H)} \widetilde{\mathcal{U}}^{(k)}\right)\right|_{\Gamma} \\
& +\left.\sum_{k \in \mathcal{N}_{2}} \eta^{(k)}\left[\overline{\mathcal{B}}_{1,0}^{(H)}\left(y, t, \frac{\partial}{\partial y}\right)-\overline{\mathcal{B}}_{1,0}^{(H)}\left(\xi^{(k)}, 0, \frac{\partial}{\partial y}\right)\right] \widetilde{\mathcal{U}}^{(k)}\right|_{\Gamma} \\
& +\sum_{k \in \mathcal{N}_{2}} \eta^{(k)} Z_{k}\left[\overline{\mathcal{B}}_{1,0}^{(H)(k)}\left(\mathbf{0}, 0, \frac{\partial}{\partial z}-\nabla F_{\xi^{(k)}} \frac{\partial}{\partial z_{3}}\right)-\overline{\mathcal{B}}_{1,0}^{(H)(k)}\left(\mathbf{0}, 0, \frac{\partial}{\partial z}\right)\right] \\
& \times\left. R^{(k)}\left(Z_{k}^{-1} \zeta^{(k)} \mathbf{k}_{1}, Z_{k}^{-1} \zeta^{(k)} \mathbf{k}_{2}\right)\right|_{\Gamma} \\
\mathcal{T}_{3} \mathcal{H} \equiv & \left.\overline{\mathcal{B}}_{2,0}^{(L)} \mathcal{R} \mathcal{H}\right|_{\Gamma_{b}}+\left.\sum_{k \in \mathcal{N}_{3}}\left(\mathcal{B}_{2,0}^{(H)} \eta^{(k)} \widetilde{\mathcal{U}}^{(k)}-\eta^{(k)} \overline{\mathcal{B}}_{2,0}^{(H)} \widetilde{\mathcal{U}}^{(k)}\right)\right|_{\Gamma_{b}} \\
& +\left.\sum_{k \in \mathcal{N}_{3}} \eta^{(k)}\left[\overline{\mathcal{B}}_{2,0}^{(H)}\left(y, t, \frac{\partial}{\partial y}\right)-\overline{\mathcal{B}}_{2,0}^{(H)}\left(\xi^{(k)}, 0, \frac{\partial}{\partial y}\right)\right] \widetilde{\mathcal{U}}^{(k)}\right|_{\Gamma_{b}} \\
& +\sum_{k \in \mathcal{N}_{3}} \eta^{(k)} Z_{k}\left[\overline{\mathcal{B}}_{2,0}^{(H)(k)}\left(\mathbf{0}, 0, \frac{\partial}{\partial z}-\nabla_{3} b_{\xi^{(k)}} \frac{\partial}{\partial z_{3}}\right)-\overline{\mathcal{B}}_{2,0}^{(H)(k)}\left(\mathbf{0}, 0, \frac{\partial}{\partial z}\right)\right] \\
& \times\left. R^{(k)}\left(Z_{k}^{-1} \zeta^{(k)} \mathbf{k}_{1}, Z_{k}^{-1} \zeta^{(k)} \mathbf{k}_{3}\right)\right|_{\Gamma_{b}}
\end{aligned}
$$

and $\overline{\mathcal{B}}_{i, 0}^{(L)}(i=1,2)$ are the operators without the derivative terms in $\mathcal{B}_{i, 0}(i=1,2)$, respectively. Thus, (5.9) holds with

$$
\mathcal{T H}=\left(\mathcal{T}_{1} \mathcal{H}, \mathcal{T}_{2} \mathcal{H}, \mathcal{T}_{3} \mathcal{H}\right)^{\mathrm{T}}
$$

Take $T_{1}=\lambda \chi^{2}$ with a certain number $\chi>0$. Then, by combining (5.14)-(5.16), and taking the regularity of $\theta(\cdot)$ into consideration, we have the following estimate [32]:

$$
\|\mathcal{T} \mathcal{H}\|_{\mathcal{W}_{\mathcal{H}}\left(T_{1}\right)} \leq C \phi_{01}(\chi, \lambda)\|\mathcal{H}\|_{\mathcal{W}_{\mathcal{H}}\left(T_{1}\right)},
$$

where $\phi_{01}$ is a homogeneous polynomial.

In a similar manner, we have (5.10) with

$$
\begin{aligned}
& W \widetilde{\mathcal{U}} \equiv \mathcal{R} \mathcal{A}_{1} \widetilde{\mathcal{U}}+\sum_{k \in \mathcal{N}_{1}} \eta^{(k)} R^{(k)}\left[\zeta^{(k)} \mathcal{L}_{0} \widetilde{\mathcal{U}}-\mathcal{L}_{0} \zeta^{(k)} \tilde{\mathcal{U}}\right]+\sum_{k \in \mathcal{N}_{1}} \eta^{(k)} R^{(k)}\left[\left\{\mathcal{L}_{0}\left(y, t, \frac{\partial}{\partial y}\right)-\mathcal{L}_{0}\left(\xi^{(k)}, 0, \frac{\partial}{\partial y}\right)\right\} \zeta^{(k)} \tilde{\mathcal{U}}\right] \\
& +\sum_{k \in \mathcal{N}_{2}} \eta^{(k)} Z_{k} R^{(k)}\left[Z_{k}^{-1}\left(\zeta^{(k)} \mathcal{L}_{0} \widetilde{\mathcal{U}}-\mathcal{L}_{0} \zeta^{(k)} \widetilde{\mathcal{U}}\right),\left.Z_{k}^{-1}\left(\zeta^{(k)} \overline{\mathcal{B}}_{1,0}^{(H)(k)} \widetilde{\mathcal{U}}-\overline{\mathcal{B}}_{1,0}^{(H)(k)} \zeta^{(k)} \widetilde{\mathcal{U}}\right)\right|_{\Gamma}\right] \\
& +\sum_{k \in \mathcal{N}_{3}} \eta^{(k)} Z_{k} R^{(k)}\left[Z_{k}^{-1}\left(\zeta^{(k)} \mathcal{L}_{0} \widetilde{\mathcal{U}}-\mathcal{L}_{0} \zeta^{(k)} \widetilde{\mathcal{U}}\right),\left.Z_{k}^{-1}\left(\zeta^{(k)} \overline{\mathcal{B}}_{2,0}^{(H)(k)} \widetilde{\mathcal{U}}-\overline{\mathcal{B}}_{2,0}^{(H)(k)} \zeta^{(k)} \widetilde{\mathcal{U}}\right)\right|_{\Gamma_{b}}\right]+\sum_{j=2}^{3} \widetilde{\Phi}_{j},
\end{aligned}
$$


with

$$
\begin{aligned}
& \widetilde{\Phi}_{2}= \sum_{k \in \mathcal{N}_{2}} \eta^{(k)} Z_{k} R^{(k)}\left[Z_{k}^{-1}\left\{\mathcal{L}_{0}\left(y, t, \frac{\partial}{\partial y}\right)-\mathcal{L}_{0}\left(\xi^{(k)}, 0, \frac{\partial}{\partial y}\right)\right\} \zeta^{(k)} \tilde{\mathcal{U}}\right. \\
&\left.\left.Z_{k}^{-1}\left\{\overline{\mathcal{B}}_{1,0}^{(H)(k)}\left(\mathbf{0}, t, \frac{\partial}{\partial y}\right)-\overline{\mathcal{B}}_{1,0}^{(H)(k)}\left(\mathbf{0}, 0, \frac{\partial}{\partial y}\right)\right\} \zeta^{(k)} \tilde{\mathcal{U}}\right|_{\Gamma}\right] \\
&+\sum_{k \in \mathcal{N}_{2}} \eta^{(k)} Z_{k} R^{(k)}\left[\left\{\mathcal{L}_{0}^{(k)}\left(\mathbf{0}, 0, \frac{\partial}{\partial z}-\nabla_{3} F \frac{\partial}{\partial z_{3}}\right)-\mathcal{L}_{0}^{(k)}\left(\mathbf{0}, 0, \frac{\partial}{\partial z}\right)\right\} Z_{k}^{-1} \zeta^{(k)} \tilde{\mathcal{U}}\right. \\
&\left.\left.\left\{\overline{\mathcal{B}}_{1,0}^{(H)(k)}\left(\mathbf{0}, 0, \frac{\partial}{\partial z}-\nabla_{3} F \frac{\partial}{\partial z_{3}}\right)-\overline{\mathcal{B}}_{1,0}^{(H)(k)}\left(\mathbf{0}, 0, \frac{\partial}{\partial z}\right)\right\} Z_{k}^{-1} \zeta^{(k)} \tilde{\mathcal{U}}\right|_{z_{3}=0}\right] \\
& \widetilde{\Phi}_{3}= \sum_{k \in \mathcal{N}_{3}} \eta^{(k)} Z_{k} R^{(k)}\left[Z_{k}^{-1}\left\{\mathcal{L}_{0}\left(y, t, \frac{\partial}{\partial y}\right)-\mathcal{L}_{0}\left(\xi^{(k)}, 0, \frac{\partial}{\partial y}\right)\right\} \zeta^{(k)} \tilde{\mathcal{U}}\right. \\
&\left.\left.Z_{k}^{-1}\left\{\overline{\mathcal{B}}_{2,0}^{(H)(k)}\left(\mathbf{0}, t, \frac{\partial}{\partial y}\right)-\overline{\mathcal{B}}_{2,0}^{(H)(k)}\left(\mathbf{0}, 0, \frac{\partial}{\partial y}\right)\right\} \zeta^{(k)} \tilde{\mathcal{U}}\right|_{\Gamma}\right] \\
&+\sum_{k \in \mathcal{N}_{3}} \eta^{(k)} Z_{k} R^{(k)}\left[\left\{\mathcal{L}_{0}^{(k)}\left(\mathbf{0}, 0, \frac{\partial}{\partial z}-\nabla_{3} b \frac{\partial}{\partial z_{3}}\right)-\mathcal{L}_{0}^{(k)}\left(\mathbf{0}, 0, \frac{\partial}{\partial z}\right)\right\} Z_{k}^{-1} \zeta^{(k)} \tilde{\mathcal{U}},\right. \\
&\left.\left.\left\{\overline{\mathcal{B}}_{2,0}^{(H)(k)}\left(\mathbf{0}, 0, \frac{\partial}{\partial z}-\nabla_{3} b \frac{\partial}{\partial z_{3}}\right)-\overline{\mathcal{B}}_{2,0}^{(H)(k)}\left(\mathbf{0}, 0, \frac{\partial}{\partial z}\right)\right\} Z_{k}^{-1} \zeta^{(k)} \tilde{\mathcal{U}}\right|_{z_{3}=0}\right]
\end{aligned}
$$

Then, by virtue of Lemma 5.4, we easily have the estimate

$$
\|W \widetilde{\mathcal{U}}\|_{\mathcal{W}_{\mathcal{U}}^{\prime}\left(T_{1}\right)} \leq C \phi_{02}(\lambda, \chi)\|\widetilde{\mathcal{U}}\|_{\mathcal{W}_{\mathcal{U}}^{\prime}\left(T_{1}\right)}
$$

with a homogeneous polynomial $\phi_{02}$. The estimates (5.17)-(5.18) lead us to conclude that both $\mathcal{T}$ and $\mathcal{W}$ are bounded operators in the spaces $\mathcal{W}_{\mathcal{H}}\left(T_{1}\right)$ and $\mathcal{W}_{\mathcal{U}}^{\prime}\left(T_{1}\right)$, respectively, and their norms are small if we take $T_{1}$ sufficiently small.

\subsubsection{Existence of operator $\mathcal{A}^{-1}$}

From the arguments above, it is possible to make the operator norms of $\mathcal{T}$ and $W$ satisfy

$$
\|\mathcal{T}\|_{\mathcal{L}\left(\mathcal{W}_{\mathcal{H}}\left(T_{1}\right): \mathcal{W}_{\mathcal{H}}\left(T_{1}\right)\right)}<1, \quad\|W\|_{\mathcal{L}\left(\mathcal{W}_{\mathcal{U}}^{\prime}\left(T_{1}\right): \mathcal{W}_{\mathcal{U}}^{\prime}\left(T_{1}\right)\right)}<1
$$

by taking $T_{1}$ small enough, where

$$
\|\cdot\|_{\mathcal{L}\left(\mathcal{W}_{A}\left(T_{1}\right): \mathcal{W}_{B}\left(T_{1}\right)\right)}
$$

is the operator norm of a linear operator from a function space $\mathcal{W}_{A}\left(T_{1}\right)$ into $\mathcal{W}_{B}\left(T_{1}\right)$.

Then, by virtue of the contraction mapping principle, it is possible to conclude that there exist $(I+\mathcal{T})^{-1}$ and $(I+W)^{-1}$. Then, by replacing $\mathcal{H}$ in $(5.9)$ by $(I+\mathcal{T})^{-1} \mathcal{H}$, and applying $(I+W)^{-1}$ to both sides of $(5.10)$, we have

$$
\begin{aligned}
& \mathcal{A R}(I+\mathcal{T})^{-1} \mathcal{H}=\mathcal{H} \\
& (I+W)^{-1} \mathcal{R} A \widetilde{\mathcal{U}}=\widetilde{\mathcal{U}}
\end{aligned}
$$


This means that $\mathcal{A}^{-1}=\mathcal{R}(I+\mathcal{T})^{-1}=(I+W)^{-1} \mathcal{R}$ exists on the time interval $\left(0, T_{1}\right)$. This is equivalent to the boundedness of the operator $\mathcal{A}^{-1}$, which is obvious by virtue of the estimate

$$
\left\|\mathcal{A}^{-1}\right\|_{\mathcal{L}\left(\mathcal{W}_{\mathcal{H}}\left(T_{1}\right): \mathcal{W}_{\mathcal{U}}^{\prime}\left(T_{1}\right)\right)} \leq\left\|(I+\mathcal{T})^{-1}\right\|_{\mathcal{L}\left(\mathcal{W}_{\mathcal{H}}\left(T_{1}\right): \mathcal{W}_{\mathcal{H}}\left(T_{1}\right)\right)}\|\mathcal{R}\|_{\mathcal{L}\left(\mathcal{W}_{\mathcal{H}}\left(T_{1}\right): \mathcal{W}_{\mathcal{U}}^{\prime}\left(T_{1}\right)\right)}
$$

If we take $T_{1}$ so small, say, $T_{10}$, that $\|\mathcal{T}\|_{\mathcal{W}_{\mathcal{H}}\left(T_{10}\right)} \leq \frac{1}{2}$, then $\left\|(I+\mathcal{T})^{-1}\right\|_{\mathcal{L}\left(\mathcal{W}_{\mathcal{H}}\left(T_{10}\right): \mathcal{W}_{\mathcal{H}}\left(T_{10}\right)\right)} \leq 2$, and

$$
\left\|\mathcal{A}^{-1}\right\|_{\mathcal{L}\left(\mathcal{W}_{\mathcal{H}}\left(T_{10}\right): \mathcal{W}_{\mathcal{U}}^{\prime}\left(T_{10}\right)\right)} \leq 2\|\mathcal{R}\|_{\mathcal{L}\left(\mathcal{W}_{\mathcal{H}}\left(T_{10}\right): \mathcal{W}_{\mathcal{U}}^{\prime}\left(T_{10}\right)\right)}
$$

the right-hand side of which is estimated by Lemma 5.4. Finally, we refer to the extension of the time interval. It is possible to consider the problem on the time interval $\left(T_{10}, 2 T_{10}\right)$, which yields the same existence result and the estimate of the solution. Iterating this process until the time interval reaches $T_{1}$, we arrive at the desired result. This completes the proof of Theorem 5.2.

\subsection{Problem for $F$}

In this subsection, we consider linear problem for $F$ with provided data on the time interval $\left(0, T_{53}\right)$.

$$
\left\{\begin{array}{l}
\frac{\partial F}{\partial t}-\mathcal{L}_{4, \breve{\mathcal{U}}, f} F=l_{4} \quad \text { in } \mathbf{R}_{T_{53}}^{2} \\
\left.F\right|_{t=0}=F_{0} \quad \text { on } \mathbf{R}^{2}
\end{array}\right.
$$

Note that the operator $\mathcal{L}_{4, \breve{\mathcal{U}}, f}$ is uniformly elliptic on a short time interval under the condition (v) in Theorem 3.1. Due to the classical result of the Cauchy problem for the linear partial differential equation of parabolic type, we have $F \in W_{2}^{\frac{5}{2}+l, \frac{5}{4}+\frac{l}{2}}\left(\mathbf{R}_{T_{53}}^{2}\right)$ and

$$
\|F\|_{W_{2}^{\frac{5}{2}+l, \frac{5}{4}+\frac{l}{2}}\left(\mathbf{R}_{T_{53}}^{2}\right)} \leq C_{51}\left(\left\|l_{4}\right\|_{W_{2}^{\frac{1}{2}+l, \frac{1}{4}+\frac{l}{2}}\left(\mathbf{R}_{T_{53}}^{2}\right)}+\left\|F_{0}\right\|_{W_{2}^{\frac{3}{2}+l}\left(\mathbf{R}^{2}\right)}\right),
$$

with $C_{51}>0$ depending on $\|\mathbf{w}\|_{W_{2}^{3+l, \frac{3+l}{2}}\left(\Omega_{T_{53}}\right)},\|f\|_{W_{2}^{\frac{5}{2}+l, \frac{5}{4}+\frac{l}{2}}\left(\mathbf{R}_{T_{53}}^{2}\right)},\|s\|_{\bar{W}_{2}^{3+l, \frac{3+l}{2}}\left(\Omega_{T_{53}}\right)}$ and $\|\sigma\|_{\bar{W}_{2}^{3+l, \frac{3+l}{2}}\left(\Omega_{T_{53}}\right)}$.

\section{Nonlinear problem (Proof of Theorem 3.1)}

In this section, we consider the original nonlinear problem by using the iteration method. This section is divided into three subsections. In the first subsection, we construct the successive approximation and estimate the right-hand side of each problem. In addition, we show the well-definedness of the successive approximation on a short time interval by using some inequalities. The second subsection provides the convergence of the successive approximation by showing they form the Cauchy sequence. Some sort of the boundedness of the temperature and salinity will be shown in the final subsection.

\subsection{Well-posedness of successive approximation}

Here we solve the problem (3.5)-(3.7) by the method of successive approximations taking $\left(\mathcal{U}_{(m)}^{\prime}, \mathbf{P}_{(m)}, F_{(m)}\right) \equiv$ $\left(\mathbf{0}, T_{0}, S_{0}, \nabla p_{u}, F_{0}\right), \mathcal{R}_{(m)}=g\left(\tilde{\varrho}_{(m)} a^{33}\left(F_{0}\right)\right)^{-1}\left|\frac{\partial \overline{\mathbf{v}}_{0}}{\partial y_{3}}\right|^{-2}$ for $m=0$, and for arbitrary $T_{1}>0$, let $\tilde{p}_{(m)}$ and 
$\tilde{\varrho}_{(m)}=\tilde{\varrho}_{(m)}(y, t) \equiv \varrho\left(\tilde{p}_{(m)}(y, t), \tilde{T}_{(m)}(y, t), \tilde{S}_{(m)}(y, t)\right) \equiv \varrho\left(\mathcal{V}_{(m)}\right)$ satisfy for $m \geq 0$

$$
\left\{\begin{array}{l}
a_{(m)}^{33} \frac{\partial \tilde{p}_{(m)}}{\partial y_{3}}=-g \tilde{\varrho}_{(m)} \quad \text { in } \Omega_{T_{1}} \\
\left.\tilde{p}_{(m)}\right|_{y_{3}=F_{0}\left(y^{\prime}\right)}=p_{0}\left(y^{\prime}, t\right) \quad \text { on } \Gamma_{b T_{1}}
\end{array}\right.
$$

where $a_{(m)}^{33}=a^{33}\left(F_{(m)}\right)$. In the following, we also use a notation $\mathcal{M}_{(m)}=\left(\tilde{T}_{(m)}, \tilde{S}_{(m)}\right)^{\mathrm{T}}$.

Defining $\left(\mathcal{U}_{(m+1)}, \mathbf{P}_{(m+1)}, F_{(m+1)}\right)=\left(\mathbf{u}_{(m+1)}^{\prime}, \tilde{T}_{(m+1)}, \tilde{S}_{(m+1)}, \mathbf{P}_{(m+1)}, F_{(m+1)}\right)(m=0,1,2, \ldots)$ as a solution to the following problem (6.3)-(6.5) provided that $\left(\mathcal{U}^{(m)}, u_{3(m)}, F_{(m)}\right)$ is given in such a way that

$$
C_{0}\left(T_{1}\right)\left\{\left\|\mathbf{u}_{(m)}^{\prime}\right\|_{W_{2}^{3+l, \frac{3+l}{2}}\left(\Omega_{T_{1}}\right)}+\sum_{1 \leq 2 \chi_{1}+\left|\chi_{2}\right| \leq 3}\left\|D_{t}^{\chi_{1}} D_{y}^{\chi_{2}} \mathcal{M}_{(m)}\right\|_{\mathcal{W}_{\mathcal{M}}\left(T_{1}\right)}+\left\|F_{(m)}-F_{0}\right\|_{W_{2}^{\frac{5}{2}+l, \frac{5}{4}+\frac{l}{2}}\left(\mathbf{R}_{T_{1}}^{2}\right)}\right\}<\delta
$$

holds with a constant $\delta>0$ :

$$
\left\{\begin{array}{l}
\frac{\partial \mathcal{U}_{(m+1)}}{\partial t}-\mathcal{L}_{\mathcal{U}_{(m)}, F_{(m)}} \mathcal{U}_{(m+1)}=\mathcal{G}_{1, F_{(m)}} \mathcal{U}_{(m)} \equiv \mathcal{E}_{1}^{(m)}=\left(\mathcal{E}_{11}^{(m)}, \mathcal{E}_{12}^{(m)}, \mathcal{E}_{13}^{(m)}\right)^{\mathrm{T}} \quad \text { in } \Omega_{T_{1}}, \\
\mathcal{B}_{1}\left(\nabla_{F_{(m)}, 3} \mathbf{u}_{(m)}, \tilde{\varrho}_{(m)}, \nabla_{F_{(m)}, 3} \tilde{\varrho}_{(m)}\right) \mathcal{U}_{(m+1)}=\bar{\tau}_{1}^{(m)} \quad \text { on } \Gamma_{T_{1}}, \\
\mathcal{B}_{2}\left(\nabla_{F_{(m)}, 3} \mathbf{u}_{(m)}, \tilde{\varrho}_{(m)}, \nabla_{F_{(m)}, 3} \tilde{\varrho}_{(m)}\right) \mathcal{U}_{(m+1)}=\bar{\tau}_{2}^{(m)} \quad \text { on } \Gamma_{b T_{1}}, \\
\left.\mathcal{U}_{(m+1)}\right|_{t=0}=\mathcal{U}_{0}=\left(\mathbf{0}, T_{0}, S_{0}\right)^{\mathrm{T}} \quad \text { on } \Omega .
\end{array}\right.
$$

$$
\begin{aligned}
\mathbf{P}_{(m+1)}= & \nabla p_{0} \exp \left(-\int_{F_{0}}^{y_{3}} \frac{g}{a_{(m)}^{33}} \varrho_{z_{1}}\left(\mathcal{V}_{(m)}\left(y^{\prime}, z_{3}, t\right)\right)\right) \mathrm{d} z_{3} \\
& +\int_{F_{0}}^{y_{3}} \Psi_{1}^{\left(\mathcal{M}_{(m+1)}, F_{(m+1)}, \mathcal{M}_{(m)}, F_{(m)}\right)}\left(y^{\prime}, z_{3}, t\right) \exp \left(-\int_{z_{3}}^{y_{3}} \frac{g}{a_{(m)}^{33}} \varrho_{z_{1}}\left(\mathcal{V}_{(m)}\right)\left(y^{\prime}, \tau, t\right) \mathrm{d} \tau\right) \mathrm{d} z_{3} \\
\equiv & \mathcal{E}_{2}^{(m)} \text { in } \Omega_{T_{1}},
\end{aligned}
$$

$$
\left\{\begin{aligned}
\frac{\partial F_{(m+1)}}{\partial t}-\mathcal{L}_{4, \mathcal{U}_{(m)}, F_{(m)}} F_{(m+1)}= & q_{w}-\mathbf{u}_{(m)} \cdot \nabla F_{(m)}-\left(\mathbf{u}_{(m)} \cdot \nabla b+\frac{1}{a_{(m)}^{33}} \int_{F_{0}\left(y^{\prime}\right)}^{y_{3}} \nabla_{F_{(m)}} \cdot \mathbf{u}_{(m)}\left(y^{\prime}, z_{3}, t\right) \mathrm{d} z_{3}\right) \\
& +\mu_{1} \sum_{i=1}^{2} \frac{\partial}{\partial y_{i}}\left(\nabla_{F_{(m)}} \cdot \mathbf{u}_{(m+1)}\right) \cdot \nabla F_{(m)}-\sum_{i=1}^{2} \frac{\partial}{\partial y_{i}}\left(\mu_{2(m)} a_{(m)}^{33} \frac{\partial \mathbf{u}_{(m+1)}}{\partial y_{3}}\right) \\
& -\nabla \cdot\left(q_{w} \mathbf{u}_{(m)}\right)-\nabla \cdot \tau_{1} \equiv \mathcal{E}_{3}^{(m)} \quad \text { in } \mathbf{R}_{T_{1}}^{2}, \\
\left.F_{(m+1)}\right|_{t=0}=F_{0} \text { on } \mathbf{R}^{2} . &
\end{aligned}\right.
$$


where

$$
\begin{aligned}
& \mu_{2(m)}=\mu_{2}\left(\nabla_{F_{(m)}, 3} \mathbf{u}_{(m)}, \tilde{\varrho}_{(m)}, \nabla_{F_{(m)}, 3} \tilde{\varrho}_{(m)}\right), \quad \mathcal{V}_{(m)}=\left(\tilde{p}_{(m)}, \tilde{T}_{(m)}, \tilde{S}_{(m)}\right)^{\mathrm{T}} \\
& \Psi_{1}^{\left(\mathcal{M}_{(m+1)}, F_{(m+1)}, \mathcal{M}_{(m)}, F_{(m)}\right)}=-g \nabla\left(\frac{1}{a_{(m+1)}^{33}}\right)-\frac{g}{a_{(m)}^{33}}\left(\varrho_{z_{2}}\left(\mathcal{V}_{(m)}\right) \nabla \tilde{T}_{(m+1)}+\varrho_{z_{3}}\left(\mathcal{V}_{(m)}\right) \nabla \tilde{S}_{(m+1)}\right), \\
& \bar{\tau}_{1(m)}=\left(\tau_{1}-\mu_{1} \nabla_{F_{(m)}} \tilde{\tilde{\mathbf{v}}}_{0}^{\left(F_{(m)}\right)} \cdot \nabla F_{(m+1)}+\mu_{2(m)} a^{33}\left(F_{(m)}\right) \frac{\partial \tilde{\mathbf{v}}_{0}^{\left(F_{(m)}\right)}}{\partial y_{3}}+q_{w} \tilde{\overline{\mathbf{v}}}_{0}^{\left(F_{(m)}\right)},\right. \\
& \left.\tau_{2}-\mu_{3} \theta_{x_{1}}\left(\mathcal{V}_{(m)}\right) \mathbf{P}_{(m)} \cdot \nabla F_{(m+1)}-\mu_{4(m)} \theta_{x_{1}}\left(\mathcal{V}_{(m)}\right) \varrho\left(\mathcal{V}_{(m)}\right) g, 0\right)^{\mathrm{T}} \\
& \bar{\tau}_{2(m)}=\left(\tau_{3}-\mu_{1} \nabla_{F_{(m)}} \tilde{\overline{\mathbf{v}}}_{0}^{\left(F_{(m)}\right)} \cdot \nabla b+\mu_{2(m)} a^{33}\left(F_{(m)}\right) \frac{\partial \tilde{\overline{\mathbf{v}}}_{0}^{\left(F_{(m)}\right)}}{\partial y_{3}},\right. \\
& \left.-\mu_{3} \theta_{x_{1}}\left(\mathcal{V}_{(m)}\right) \mathbf{P}_{(m)} \cdot \nabla b-\mu_{4(m)} \theta_{x_{1}}\left(\mathcal{V}_{(m)}\right) \varrho\left(\mathcal{V}_{(m)}\right) g, 0\right)^{\mathrm{T}} .
\end{aligned}
$$

Note that in deriving the expression of $\mathbf{P}_{(m+1)}$, we have made use of (4.2) in Lemma 4.7. The unique existence of $\mathcal{U}_{(m+1)}$ is guaranteed by Theorem 5.2, and we shall estimate the norm of the solution. By the interpolation and Young's inequalities, it is easy to confirm [23] that for a function $f \in W_{2}^{m, \frac{m}{2}}\left(\Omega_{T_{1}}\right)$ satisfying $\left.f\right|_{t=0}=0$ in general,

$$
\|f\|_{W_{2}^{k, 0}\left(\Omega_{T_{1}}\right)}^{2} \leq \epsilon\|f\|_{W_{2}^{m, 0}\left(\Omega_{T_{1}}\right)}^{2}+C_{\epsilon} T_{1}^{2}\left\|\frac{\partial f}{\partial t}\right\|_{L_{2}\left(\Omega_{T_{1}}\right)}^{2}
$$

for any $\epsilon>0$ if $m>k$. Making use of (6.6) and Theorem 5.2, we shall estimate the right-hand sides of (6.3)-(6.5) with the aid of the following lemma. Hereafter we use notations

$$
\begin{aligned}
E_{0}(t) \equiv & \left\|\breve{\mathcal{U}}_{0}\right\|_{\mathcal{W}_{0}}+\left\|F_{0}\right\|_{W_{2}^{\frac{5}{2}+l}\left(\mathbf{R}^{2}\right)}+\sum_{i=1}^{3}\left\|\tau_{i}\right\|_{W_{2}^{\frac{3}{2}+l^{\prime \prime}, \frac{4}{3}+\frac{l^{\prime \prime}}{2}}\left(\mathbf{R}_{t}^{2}\right)}+\left\|q_{w}\right\|_{W_{2}^{\frac{1}{2}+l, \frac{1}{4}+\frac{l}{2}}\left(\mathbf{R}_{t}^{2}\right)} \\
& +\left\|p_{0}\right\|_{W_{2}^{\frac{5}{2}+l, \frac{5}{4}+\frac{l}{2}}\left(\mathbf{R}_{t}^{2}\right)}+\|b\|_{\bar{W}_{2}^{\frac{5}{2}+l}\left(\mathbf{R}^{2}\right)}
\end{aligned}
$$

and $\mathcal{R}_{(m)} \equiv \frac{g \tilde{\varrho}_{(m)}^{-1}}{a_{(m)}^{33}} \frac{\partial \tilde{\varrho}_{(m)}}{\partial y_{3}}\left|\frac{\partial \mathbf{u}_{(m)}}{\partial y_{3}}\right|^{-2}$ for $m \geq 1$

Lemma 6.1. Assume $F_{0} \in W_{2}^{\frac{5}{2}+l}\left(\mathbf{R}^{2}\right), \mathcal{U}_{0} \equiv\left(\mathbf{v}_{0}, T_{0}, S_{0}\right)^{\mathrm{T}} \in \mathcal{W}_{0}, \min \left\{\left(1+\alpha_{i} \mathcal{R}_{0}\right)\left|\frac{\partial \mathbf{v}_{0}}{\partial x_{3}}\right|,\left|\frac{\partial \mathbf{v}_{0}}{\partial x_{3}}\right|\right\} \geq c_{u}>0(i=$ $2,4,6)$. In addition, for a certain $m$ provided, let us assume that there exists $T_{61}>0$ satisfying $F_{(m)}\left(y^{\prime}, t\right)-$ $b\left(y^{\prime}\right)>c_{0}>0$ and $\min \left\{\left(1+\alpha_{i} \mathcal{R}_{(m)}\right)\left|\frac{\partial \mathbf{u}_{(m)}}{\partial y_{3}}\right|,\left|\frac{\partial \mathbf{u}_{(m)}}{\partial y_{3}}\right|\right\}>c_{u}>0(i=1,2,3)$ for $t \in\left(0, T_{61}\right]$. Then, for the 
right-hand side of (6.3)-(6.5), following estimates hold for arbitrary small $\epsilon>0$ and $t \in\left(0, T_{61}\right]$ :

$$
\begin{aligned}
& \left\|\mathcal{E}_{1}^{(m)}\right\|_{\mathcal{W}_{1}(t)}+\sum_{i=1}^{2}\left\|\bar{\tau}_{i(m)}\right\|_{\mathcal{W}_{2}(t)} \leq\left(\epsilon+C_{\epsilon} t\right)\left\{\phi_{61}\left(\left\|\mathcal{U}_{(m)}\right\|_{\mathcal{W}_{\mathcal{U}}(t)}+\left\|F_{(m)}\right\|_{W_{2}^{\frac{5}{2}+l, \frac{5}{4}+\frac{l}{2}}\left(\mathbf{R}_{t}^{2}\right)}\right)+\left\|\mathbf{P}_{(m)}\right\|_{W_{2}^{\frac{3}{2}+l, \frac{3}{4}+\frac{l}{2}}\left(\Omega_{t}\right)}\right. \\
& \left.\left.+\phi_{62}(\| \mathcal{M}]_{(m)} \|_{\mathcal{W}_{\mathcal{M}}(t)}\right)\left\|F_{(m+1)}\right\|_{W_{2}^{\frac{5}{2}+l, \frac{5}{4}+\frac{l}{2}}\left(\mathbf{R}_{t}^{2}\right)}\right\}+C_{61}(t) E_{0}(t) \\
& \left\|\mathcal{E}_{2}^{(m)}\right\|_{W_{2}^{\frac{3}{2}+l, \frac{3}{4}+\frac{l}{2}\left(\Omega_{t}\right)}} \leq \phi_{63}\left(\left\|\mathcal{M}_{(m)}\right\|_{\mathcal{W}_{\mathcal{M}}(t)}+\left\|F_{(m)}\right\|_{W_{2}^{\frac{5}{2}+l, \frac{5}{4}+\frac{l}{2}}\left(\mathbf{R}_{t}^{2}\right)}\right)\left(\left\|F_{(m+1)}\right\|_{W_{2}^{\frac{5}{2}+l, \frac{5}{4}+\frac{l}{2}}\left(\mathbf{R}_{t}^{2}\right)}+\left\|\mathcal{M}_{(m+1)}\right\|_{\mathcal{W}_{\mathcal{M}}(t)}\right) \\
& +\phi_{64}\left(\left\|F_{(m)}\right\|_{W_{2}^{\frac{5}{2}+l, \frac{5}{4}+\frac{l}{2}}\left(\mathbf{R}_{t}^{2}\right)}+\left\|\mathcal{M}_{(m)}\right\|_{\mathcal{W}_{\mathcal{M}}(t)}\right), \\
& \left\|\mathcal{E}_{3}^{(m)}\right\|_{W_{2}^{\frac{1}{2}+l, \frac{1}{4}+\frac{l}{2}}\left(\mathbf{R}_{t}^{2}\right)} \leq \phi_{65}\left(\left\|\mathcal{U}_{(m)}\right\|_{\mathcal{W}_{\mathcal{U}}\left(T_{1}\right)}+\left\|F_{(m)}\right\|_{W_{2}^{\frac{5}{2}+l, \frac{5}{4}+\frac{l}{2}}\left(\mathbf{R}_{t}^{2}\right)}\right)\left\|\mathbf{u}_{(m+1)}^{\prime}\right\|_{W_{2}^{3+l, \frac{3+l}{2}}{ }_{\left(\Omega_{t}\right)}} \\
& +\left(\epsilon+C_{\epsilon} t\right)\left[\left\{\left\|\mathbf{u}_{(m)}^{\prime}\right\|_{W_{2}^{3+l, \frac{3+l}{2}}\left(\Omega_{t}\right)}+\phi_{66}\left(\left\|F_{(m)}\right\|_{W_{2}^{\frac{5}{2}+l, \frac{5}{4}+\frac{l}{2}}\left(\mathbf{R}_{t}^{2}\right)}\right)\right\}\right. \\
& \times\left(1+\left\|F_{(m)}\right\|_{W_{2}^{\frac{5}{2}+l, \frac{5}{4}+\frac{l}{2}}\left(\mathbf{R}_{t}^{2}\right)}\right) \\
& \left.+\phi_{67}\left(\left\|F_{(m)}\right\|_{W_{2}^{\frac{5}{2}+l, \frac{5}{4}+\frac{l}{2}}\left(\mathbf{R}_{t}^{2}\right)}+\left\|\mathcal{M}_{(m)}\right\|_{\mathcal{W}_{\mathcal{M}}(t)}\right)\right]
\end{aligned}
$$

where $\phi_{6 i}(\cdot)(i=1,2, \ldots, 7)$ are positive and monotonically increasing functions of their arguments, and $C_{\epsilon}$ is a positive constant depending on $\epsilon$.

Proof. We show a part of the estimate of $\left\|\mathcal{E}_{1}^{(m)}\right\|_{\mathcal{W}_{2}(t)}$ here, especially $\left\|\mathcal{E}_{11}^{(m)}\right\|_{W_{2}^{1+l, \frac{1+l}{2}}\left(\Omega_{t}\right)}$ since other terms are estimated similarly.

$$
\begin{aligned}
\mathcal{E}_{11}^{(m)}= & -\left(\mathbf{u}_{(m)} \cdot \nabla\right) \mathbf{u}_{(m)}^{\prime}+\left(\mathbf{u}_{(m)} \cdot \nabla b+\frac{1}{a_{(m)}^{33}} \int_{F_{0}\left(y^{\prime}\right)}^{y_{3}} \nabla_{F_{(m)}} \cdot \mathbf{u}_{(m)}\left(y^{\prime}, z_{3}, t\right) \mathrm{d} z_{3}\right) a_{(m)}^{33} \frac{\partial \mathbf{u}_{(m)}^{\prime}}{\partial y_{3}} \\
& -\tilde{\mathbf{v}}_{0}^{\left(F_{(m)}\right)} \cdot \nabla_{F_{(m)}} \mathbf{u}_{(m)}^{\prime}-f \mathbf{A} \mathbf{u}_{(m)}^{\prime}-\frac{\mathbf{a}_{(m)}^{3} g}{a_{(m)}^{33}} \varrho\left(\mathcal{V}_{(m)}\right)-\left(\tilde{\overline{\mathbf{v}}}_{0}^{\left(F_{(m)}\right)} \cdot \nabla_{F_{(m)}} \tilde{\mathbf{v}}_{0}^{\left(F_{(m)}\right)}-\mathbf{v}_{0} \cdot \nabla \mathbf{v}_{0}\right) \\
& +\left\{\left(\mathbf{u}_{(m)} \cdot \nabla b+\frac{1}{a_{(m)}^{33}} \int_{F_{0}\left(y^{\prime}\right)}^{y_{3}} \nabla_{F_{(m)}} \cdot \mathbf{u}_{(m)}\left(y^{\prime}, z_{3}, t\right) \mathrm{d} z_{3}\right) a_{(m)}^{33} \frac{\partial \mathbf{u}_{(m)}^{\prime}}{\partial y_{3}}\right. \\
& \left.-\left(\mathbf{v}_{0} \cdot \nabla b+\int_{F_{0}\left(y^{\prime}\right)}^{y_{3}} \nabla \cdot \mathbf{v}_{0}\left(y^{\prime}, z_{3}, t\right) \mathrm{d} z_{3}\right) \frac{\partial \mathbf{v}_{0}}{\partial y_{3}}\right\} \\
& +\left(\mathcal{L}_{1, \mathcal{U}_{(m)}, F_{(m)}} \tilde{\overline{\mathbf{v}}}_{0}^{\left(F_{(m)}\right)}-\mathcal{L}_{1,0} \mathbf{v}_{0}\right)-f \mathbf{A}\left(\tilde{\overline{\mathbf{v}}}_{0}^{\left(F_{(m)}\right)}-\mathbf{v}_{0}\right)+M_{1}(y) .
\end{aligned}
$$


We show the estimates of some of terms in the right-hand side of the above equality. First, by virtue of the multiplicative and interpolation inequalities and Lemma 4.3, we have

$$
\begin{aligned}
\left\|\left(\mathbf{u}_{(m)} \cdot \nabla_{F_{(m)}}\right) \mathbf{u}_{(m)}^{\prime}\right\|_{W_{2}^{1+l, \frac{1+l}{2}}\left(\Omega_{t}\right)} \leq & \left(\epsilon+C_{\epsilon} t\right)\left\{\left\|\mathbf{u}_{(m)}^{\prime}\right\|_{W_{2}^{3+l, \frac{3+l}{2}}\left(\Omega_{t}\right)}+\phi\left(\left\|F_{(m)}\right\|_{W_{2}^{\frac{5}{2}+l, \frac{5}{4}+\frac{l}{2}}\left(\mathbf{R}_{t}^{2}\right)}\right)\right\} \\
& \times\left(1+\left\|F_{(m)}\right\|_{W_{2}^{\frac{5}{2}+l, \frac{5}{4}+\frac{l}{2}}\left(\mathbf{R}_{t}^{2}\right)}\right)\left\|\mathbf{u}_{(m)}^{\prime}\right\|_{W_{2}^{3+l, \frac{3+l}{2}}\left(\Omega_{t}\right)} .
\end{aligned}
$$

Next, by virtue of (4.13) of Lemma 4.10 and Lemma 4.6, we have

$$
\begin{aligned}
\| \mathcal{L}_{1, \mathcal{U}_{(m)}, F_{(m)}} \tilde{\overline{\mathbf{v}}}_{0}^{\left(F_{(m)}\right)} & -\mathcal{L}_{1,0} \mathbf{v}_{0} \|_{W_{2}^{1+l, \frac{1+l}{2}}\left(\Omega_{t}\right)} \\
\leq & \left\|\left[\mathcal{L}_{1, \mathcal{U}_{(m)}, F_{(m)}}-\mathcal{L}_{1,0}\right] \tilde{\overline{\mathbf{v}}}_{0}^{\left(F_{(m)}\right)}\right\|_{W_{2}^{1+l, \frac{1+l}{2}}\left(\Omega_{t}\right)}+\left\|\mathcal{L}_{1,0}\left(\tilde{\overline{\mathbf{v}}}_{0}^{\left(F_{(m)}\right)}-\mathbf{v}_{0}\right)\right\|_{W_{2}^{1+l, \frac{1+l}{2}}\left(\Omega_{t}\right)} \\
& \leq \phi_{0}\left(\epsilon+C_{\epsilon} t\right) \phi_{1}\left(\left\|\mathcal{U}_{(m)}\right\|_{\mathcal{W}_{\mathcal{U}}(t)}+\left\|F_{(m)}\right\|_{W_{2}^{\frac{5}{2}+l, \frac{5}{4}+\frac{l}{2}}\left(\mathbf{R}_{t}^{2}\right)}\right)
\end{aligned}
$$

for a homogeneous polynomial $\phi_{0}$ and a polynomial $\phi_{1}$. Other terms are estimated by making use of Lemmas 4.2 and 4.5 , and by combining these, we arrive at the desired result.

Now we introduce the notations

$$
E_{(m)}(t) \equiv\left\|\left(\mathcal{U}_{(m)}, \mathbf{P}_{(m)}, F_{(m)}\right)\right\|_{\mathcal{W}(t)}, \quad E_{(m)}^{\prime}(t) \equiv\left\|\mathcal{U}_{(m)}\right\|_{\mathcal{W}_{\mathcal{U}}(t)},
$$

and take $T_{61}$ such that $F_{(m)}\left(y^{\prime}, t\right)-b\left(y^{\prime}\right)>c_{0}, \min \left\{\left(1+\alpha_{i} \mathcal{R}_{(m)}\right)\left|\frac{\partial \mathbf{u}_{(m)}}{\partial y_{3}}\right|,\left|\frac{\partial \mathbf{u}_{(m)}}{\partial y_{3}}\right|\right\}>\frac{c_{u}}{2}>0$ hold for $t \in$ $\left(0, T_{61}\right]$. Then, we have the following estimates for $t \in\left(0, T_{61}\right]$ with the aid of the results of the previous section and Lemma 6.1:

$$
\begin{aligned}
& E_{(m+1)}^{\prime}(t) \leq\left(\epsilon+C_{\epsilon} t\right)\left\{\phi_{61}\left(E_{(m)}(t)\right)+\phi_{62}\left(E_{(m)}(t)\right)\left\|F_{(m+1)}\right\|_{W_{2}^{\frac{5}{2}+l, \frac{5}{4}+\frac{l}{2}}\left(\mathbf{R}_{t}^{2}\right)}\right\}+C_{62}(t) \\
&\left\|\mathbf{P}_{(m+1)}\right\|_{W_{2}^{\frac{3}{2}+l, \frac{3}{4}+\frac{l}{2}}\left(\Omega_{t}\right)} \leq \phi_{63}\left(E_{(m)}\left(T_{1}\right)\right)\left(\left\|F_{(m+1)}\right\|_{W_{2}^{\frac{5}{2}+l, \frac{5}{4}+\frac{l}{2}}\left(\mathbf{R}_{t}^{2}\right)}+\left\|\mathcal{M}_{(m+1)}\right\|_{\mathcal{W}_{\mathcal{M}}(t)}\right)+\phi_{64}\left(E_{(m)}(t)\right) \\
&\left\|F_{(m+1)}\right\|_{W_{2}^{\frac{5}{2}+l, \frac{5}{4}+\frac{l}{2}}\left(\mathbf{R}_{t}^{2}\right)} \leq \phi_{65}\left(E_{(m)}(t)\right)\left\|\mathbf{u}_{(m+1)}^{\prime}\right\|_{W_{2}^{3+l, \frac{3+l}{2}}\left(\Omega_{t}\right)}+\left(\epsilon+C_{\epsilon} t\right) \phi_{66}\left(E_{(m)}(t)\right)+E_{0}(t)
\end{aligned}
$$

First, by adding (6.9) and (6.8) multiplied by $1 / 2 \phi_{63}\left(E_{(m)}\left(T_{1}\right)\right)$, we have

$$
\begin{aligned}
\left\|F_{(m+1)}\right\|_{W_{2}^{\frac{5}{2}+l, \frac{5}{4}+\frac{l}{2}}\left(\mathbf{R}_{t}^{2}\right)}+\left\|\mathbf{P}_{(m+1)}\right\|_{W_{2}^{\frac{3}{2}+l, \frac{3}{4}+\frac{l}{2}}\left(\Omega_{t}\right)} & \\
& \leq \phi_{67}\left(E_{m}\left(T_{1}\right) E_{(m+1)}^{\prime}(t)+\left(\epsilon+C_{\epsilon} t\right) \phi_{68}\left(E_{m}(t)\right)+C_{63}\left(T_{61}\right) .\right.
\end{aligned}
$$

Then, by taking $\epsilon$ and $t$ small enough, if necessary, and by adding (6.7) and (6.10) multiplied by $1 / 2 \phi_{67}\left(E_{m}\left(T_{1}\right)\right)$, and estimating the right-hand side, we arrive at the inequality of the form

$$
E_{(m+1)}(t) \leq C_{64}\left(T_{1}\right)\left\{\left(\epsilon+C_{\epsilon} t\right) \phi_{69}\left(E_{(m)}\left(T_{61}\right)\right)+1\right\}
$$

with some $C_{64}(t) \geq 0$ depending on $t$ monotonically and increasingly. Take $M>0$ such that $E_{(m)}\left(T_{61}\right)<M$ holds. Then, take $\epsilon$ first so that

$$
\epsilon C_{64}\left(T_{1}\right) \phi_{69}(M)<M-C_{64}\left(T_{1}\right)
$$


holds, and then $T_{61} \in\left(0, T_{1}\right]$ so that

$$
C_{64}\left(T_{61}\right) C_{\epsilon} \phi_{69}(M) T_{61}<M-C_{64}\left(T_{61}\right)-\epsilon C_{64}\left(T_{61}\right) \phi_{69}(M) .
$$

Consequently we obtain $E_{(m+1)}\left(T_{61}\right)<M$ from the assumption $E_{(m)}\left(T_{61}\right)<M$. Now we show that $T_{61}$ does not depend on $m$ with the following lemma:

Lemma 6.2. For $T_{1}>0$ sufficiently small, we have the following inequality for any $m \geq 0$ :

$$
\begin{gathered}
\left|\left(1+\alpha_{i} \mathcal{R}_{(m)}(y, t)\right)\right| \frac{\partial \mathbf{u}_{(m)}}{\partial y_{3}}(y, t)\left|-\left(1+\alpha_{i} \mathcal{R}_{0}(y)\right)\right| \frac{\partial \mathbf{v}_{0}}{\partial y_{3}}(y)|| \leq C_{65} \frac{1}{2}\left(\left\|\mathcal{U}_{(m)}\right\|_{\mathcal{W}_{\mathcal{U}}(t)}+\left\|F_{(m)}\right\|_{W_{2}^{\frac{5}{2}+l, \frac{5}{4}+\frac{l}{2}}\left(\mathbf{R}_{t}^{2}\right)}\right) \\
\forall(y, t) \in \Omega_{T_{1}} .
\end{gathered}
$$

Proof. This lemma is shown by direct calculations with the aid of the Sobolev embedding theorem, and we omit the proof of it (the function space $\mathcal{W}_{\mathcal{U}}(t)$ is defined in the statement of Lem. 4.10).

Due to Lemma 6.2, we have $\left(1+\alpha_{i} \mathcal{R}_{(m)}(y, t)\right)\left|\frac{\partial \mathbf{u}_{(m)}}{\partial y_{3}}(y, t)\right|>\frac{c_{u}}{2}$ for $t \in\left(0, \frac{c_{u}}{2 C_{65} M}\right)$. Thus, by taking $T_{61}$ small enough, it does not depend on $m$. By induction $\left\{\mathcal{U}_{(m)}, \mathbf{P}_{(m)}, F_{(m)}\right\}_{m=0}^{\infty}$ is well defined in $\mathcal{W}\left(T_{61}\right)$ satisfying (5.8) with $(\mathbf{w}, \sigma, s, f)$ replaced by $\left(\mathbf{u}_{(m)}, T_{(m)}, S_{(m)}, F_{(m)}\right)$ and $E_{(m)}\left(T_{61}\right)<M$ for $m=0,1,2, \ldots$

\subsection{Convergence of the successive approximation}

In this subsection, we show the convergence of the successive approximation. Subtract (6.3)-(6.5) with $m$ replaced by $m-1$ from itself. Denoting $\widetilde{\mathcal{U}}_{(m)} \equiv \mathcal{U}_{(m)}-\mathcal{U}_{(m-1)}, \tilde{\mathbf{u}}_{(m)}^{\prime}=\mathbf{u}_{(m)}^{\prime}-\mathbf{u}_{(m-1)}^{\prime}, \widetilde{\mathbf{P}}_{(m)} \equiv \mathbf{P}_{(m)}-\mathbf{P}_{(m-1)}$, $\widetilde{F}_{(m)} \equiv F_{(m)}-F_{(m-1)}$ for $m \geq 0$, we consider the following problem:

$$
\begin{aligned}
& \left\{\begin{array}{c}
\frac{\partial \widetilde{\mathcal{U}}_{(m+1)}}{\partial t}-\mathcal{L}_{\mathcal{U}_{(m)}, F_{(m)}} \tilde{\mathcal{U}}_{(m+1)}=\left[\mathcal{E}_{1}^{(m)}-\mathcal{E}_{1}^{(m-1)}\right]+\left[\mathcal{L}_{\mathcal{U}_{(m)}, F_{(m)}}-\mathcal{L}_{\mathcal{U}_{(m-1)}, F_{(m-1)}}\right] \mathcal{U}_{(m)} \quad \text { in } \Omega_{T_{61}}, \\
\mathcal{B}_{1}\left(\nabla_{F_{(m)}, 3} \mathbf{u}_{(m)}, \tilde{\varrho}_{(m)}, \nabla_{F_{(m)}, 3} \tilde{\varrho}_{(m)}\right) \tilde{\mathcal{U}}_{(m+1)} \\
=\left[\bar{\tau}_{1(m)}-\bar{\tau}_{1(m-1)}\right]+\left[\mathcal{B}_{1}\left(\nabla_{F_{(m)}, 3} \mathbf{u}_{(m)}, \tilde{\varrho}_{(m)}, \nabla_{F_{(m)}, 3} \tilde{\varrho}_{(m)}\right)\right. \\
\left.-\mathcal{B}_{1}\left(\nabla_{F_{(m-1)}, 3} \mathbf{u}_{(m-1)}, \tilde{\varrho}_{(m-1)}, \nabla_{F_{(m-1)}, 3} \tilde{\varrho}_{(m-1)}\right)\right] \mathcal{U}_{(m)} \quad \text { on } \Gamma_{T_{61}}, \\
\mathcal{B}_{2}\left(\nabla_{F_{(m)}, 3} \mathbf{u}_{(m)}, \tilde{\varrho}_{(m)}, \nabla_{F_{(m)}, 3} \tilde{\varrho}_{(m)}\right) \tilde{\mathcal{U}}_{(m+1)} \\
=\left[\bar{\tau}_{2(m)}-\bar{\tau}_{2(m-1)}\right]+\left[\mathcal{B}_{2}\left(\nabla_{F_{(m)}, 3} \mathbf{u}_{(m)}, \tilde{\varrho}_{(m)}, \nabla_{F_{(m)}, 3} \tilde{\varrho}_{(m)}\right)\right. \\
\left.-\mathcal{B}_{2}\left(\nabla_{F_{(m-1)}, 3} \mathbf{u}_{(m-1)}, \tilde{\varrho}_{(m-1)}, \nabla_{F_{(m-1)}, 3} \tilde{\varrho}_{(m-1)}\right)\right] \mathcal{U}_{(m)} \quad \text { on } \Gamma_{b T_{61}}, \\
\left.\tilde{\mathcal{U}}_{(m+1)}\right|_{t=0}=\mathbf{0} \quad \text { on } \Omega,
\end{array}\right. \\
& \widetilde{\mathbf{P}}_{(m+1)}=\mathcal{E}_{2}^{(m)}-\mathcal{E}_{2}^{(m-1)} \quad \text { in } \Omega_{T_{61}}, \\
& \left\{\begin{array}{l}
\frac{\partial \widetilde{F}_{(m+1)}}{\partial t}-\mathcal{L}_{4, \mathcal{U}_{(m-1)}, F_{(m-1)}} \widetilde{F}_{(m+1)}=\left[\mathcal{E}_{3}^{(m)}-\mathcal{E}_{3}^{(m-1)}\right]+\left[\mathcal{L}_{4, \mathcal{U}_{(m)}, F_{(m)}}-\mathcal{L}_{4, \mathcal{U}_{(m-1)}, F_{(m-1)}}\right] F_{(m)} \quad \text { in } \mathbf{R}_{T_{61}}^{2}, \\
\left.\widetilde{F}_{(m+1)}\right|_{t=0}=0 \quad \text { on } \mathbf{R}^{2} .
\end{array}\right.
\end{aligned}
$$


Lemma 6.3. Under the assumptions of Lemma 6.1, following estimates hold for $\epsilon>0$ and $t \in\left(0, T_{61}\right)$ :

$$
\begin{aligned}
& \left\|\mathcal{E}_{1}^{(m)}-\mathcal{E}_{1}^{(m-1)}\right\|_{\mathcal{W}_{1}(t)}+\sum_{i=1}^{2}\left\|\bar{\tau}_{i(m)}-\bar{\tau}_{i(m-1)}\right\|_{\mathcal{W}_{i+1}(t)} \\
& \leq\left(\epsilon+C_{\epsilon} t\right)\left[\phi_{610}\left(\sum_{i=m-1}^{m}\left(\left\|\mathcal{U}_{(i)}\right\|_{\mathcal{W}_{\mathcal{U}}(t)}+\left\|F_{(i)}\right\|_{W_{2}^{\frac{5}{2}+l, \frac{5}{4}+\frac{l}{2}}\left(\mathbf{R}_{t}^{2}\right)}\right)+\left\|\mathbf{P}_{(m-1)}\right\|_{W_{2}^{\frac{3}{2}+l, \frac{3}{4}+\frac{l}{2}}\left(\Omega_{t}\right)}\right)\right. \\
& \times\left(\left\|\widetilde{\mathcal{U}}_{(m)}\right\|_{\mathcal{W}_{\mathcal{U}}^{\prime}(t)}+\left\|\widetilde{\mathbf{P}}_{(m)}\right\|_{W_{2}^{\frac{3}{2}+l, \frac{3}{4}+\frac{l}{2}}\left(\Omega_{t}\right)}+\left\|\widetilde{F}_{(m)}\right\|_{W_{2}^{\frac{5}{2}+l, \frac{5}{4}+\frac{l}{2}}\left(\mathbf{R}_{t}^{2}\right)}\right) \\
& +\phi_{611}\left(\sum_{i=m-1}^{m+1}\left\|F_{(i)}\right\|_{W_{2}^{\frac{5}{2}+l, \frac{5}{4}+\frac{l}{2}}\left(\mathbf{R}_{t}^{2}\right)}\right)\left\|\widetilde{F}_{(m)}\right\|_{W_{2}^{\frac{5}{2}+l, \frac{5}{4}+\frac{l}{2}}\left(\mathbf{R}_{t}^{2}\right)} \\
& \left.+\phi_{612}\left(\left\|F_{(m-1)}\right\|_{W_{2}^{\frac{5}{2}+l, \frac{5}{4}+\frac{l}{2}}\left(\mathbf{R}_{t}^{2}\right)}\right)\left\|\widetilde{F}_{(m+1)}\right\|_{W_{2}^{\frac{5}{2}+l, \frac{5}{4}+\frac{l}{2}}\left(\mathbf{R}_{t}^{2}\right)}\right] \\
& \left\|\mathcal{E}_{2}^{(m)}-\mathcal{E}_{2}^{(m-1)}\right\|_{W_{2}^{\frac{3}{2}+l, \frac{3}{4}+\frac{l}{2}}\left(\Omega_{t}\right)} \leq \phi_{613}\left(\sum_{i=m-1}^{m}\left\|\mathcal{M}_{(i)}\right\|_{\mathcal{W}_{\mathcal{M}}(t)}+\left\|F_{(i)}\right\|_{W_{2}^{\frac{5}{2}+l, \frac{5}{4}+\frac{l}{2}}\left(\mathbf{R}_{t}^{2}\right)}\right) \\
& \times\left(\left\|\widetilde{\mathcal{M}}_{(m+1)}\right\|_{\mathcal{W}_{\mathcal{M}(t)}}+\left\|\widetilde{F}_{(m+1)}\right\|_{W_{2}^{\frac{5}{2}+l, \frac{5}{4}+\frac{l}{2}}\left(\mathbf{R}_{t}^{2}\right)}\right) \\
& +\left(\epsilon+C_{\epsilon} t\right) \phi_{614}\left(\sum_{i=m-1}^{m+1}\left\|\mathcal{M}_{(i)}\right\|_{\mathcal{W}_{\mathcal{M}}(t)}+\left\|F_{(i)}\right\|_{W_{2}^{\frac{5}{2}+l, \frac{5}{4}+\frac{l}{2}}\left(\mathbf{R}_{t}^{2}\right)}\right) \\
& \times\left(\left\|\widetilde{F}_{(m)}\right\|_{W_{2}^{\frac{5}{2}+l, \frac{5}{4}+\frac{l}{2}}\left(\mathbf{R}_{t}^{2}\right)}+\left\|\widetilde{\mathcal{M}}_{(m)}\right\|_{\mathcal{W}_{\mathcal{M}}(t)}\right), \\
& \left\|\mathcal{E}_{3}^{(m)}-\mathcal{E}_{3}^{(m-1)}\right\|_{W_{2}^{\frac{1}{2}+l, \frac{1}{4}+\frac{l}{2}}\left(\mathbf{R}_{t}^{2}\right)} \leq \phi_{615}\left(\left\|\mathcal{M}_{(m)}\right\|_{W_{2}^{3+l, \frac{3+l}{2}}\left(\Omega_{t}\right)}+\left\|F_{(m)}\right\|_{W_{2}^{\frac{5}{2}+l, \frac{5}{4}+\frac{l}{2}}\left(\mathbf{R}_{t}^{2}\right)}\right) \\
& \times\left\{\left\|\tilde{\mathbf{u}}_{(m+1)}^{\prime}\right\|_{W_{2}^{3+l, \frac{3+l}{2}}\left(\Omega_{t}\right)}+\left(\epsilon+C_{\epsilon} t\right)\left\|\widetilde{F}_{(m+1)}\right\|_{W_{2}^{\frac{5}{2}+l, \frac{5}{4}+\frac{l}{2}}\left(\mathbf{R}_{t}^{2}\right)}\right\} \\
& +\left(\epsilon+C_{\epsilon} t\right) \phi_{616}\left(\sum_{i=m-1}^{m}\left\|\mathcal{U}_{(i)}\right\|_{\mathcal{W}_{\mathcal{U}}(t)}+\left\|F_{(i)}\right\|_{W_{2}^{\frac{5}{2}+l, \frac{5}{4}+\frac{l}{2}}\left(\mathbf{R}_{t}^{2}\right)}\right) \\
& \times\left(\left\|\tilde{\mathbf{u}}_{(m)}^{\prime}\right\|_{W_{2}^{3+l, \frac{3+l}{2}}\left(\Omega_{t}\right)}+\left\|\widetilde{\mathcal{M}}_{(m)}\right\|_{W_{2}^{3+l, \frac{3+l}{2}}\left(\Omega_{t}\right)}+\left\|\tilde{F}_{(m)}\right\|_{W_{2}^{\frac{5}{2}+l, \frac{5}{4}+\frac{l}{2}}\left(\mathbf{R}_{t}^{2}\right)}\right),
\end{aligned}
$$

where $\widetilde{\mathcal{M}}_{(m)}=\mathcal{M}_{(m)}-\mathcal{M}_{(m-1)}, C_{\epsilon}$ is a positive constant depending on $\epsilon$, and $\phi_{6 i}(\cdot)(i=10,11, \ldots, 16)$ are increasing functions of their arguments.

Proof. This lemma is shown with the aid of Lemmas 4.1-4.3 and 4.6 and multiplicative inequalities, and we omit the detail here.

Let us define

$$
\mathcal{W}^{\prime}\left(T_{61}\right) \equiv \mathcal{W}_{\mathcal{U}}^{\prime}\left(T_{61}\right) \times W_{2}^{\frac{3}{2}+l, \frac{3}{4}+\frac{l}{2}}\left(\Omega_{T_{61}}\right) \times W_{2}^{\frac{5}{2}+l, \frac{5}{4}+\frac{l}{2}}\left(\mathbf{R}_{T_{61}}^{2}\right),
$$


and $\widetilde{E}_{(m)}(t) \equiv\left\|\left(\widetilde{\mathcal{U}}_{(m)}, \tilde{P}_{(m)}, \widetilde{F}_{(m)}\right)\right\|_{\mathcal{W}^{\prime}(t)}, \quad \widetilde{E}_{(m)}^{\prime}(t) \equiv\left\|\widetilde{\mathcal{U}}_{(m)}\right\|_{\mathcal{W}_{\mathcal{U}}^{\prime}(t)}$. From Lemma 6.3 , we have the following inequality for any $t \in\left(0, T_{61}\right)$ :

$$
\begin{aligned}
& \widetilde{E}_{(m+1)}^{\prime}(t) \leq\left(\epsilon+C_{\epsilon} t\right)\left[\phi_{617}(t)\left(E_{(m-1)}(t)+E_{(m)}(t)+E_{(m+1)}(t)\right) \widetilde{E}_{(m)}(t)\right. \\
& \left.+\phi_{612}\left(\left\|F_{(m-1)}\right\|_{W_{2}^{\frac{5}{2}+l, \frac{5}{4}+\frac{l}{2}}\left(\mathbf{R}_{t}^{2}\right)}\right)\left\|\widetilde{F}_{(m+1)}\right\|_{W_{2}^{\frac{5}{2}+l, \frac{5}{4}+\frac{l}{2}}\left(\mathbf{R}_{t}^{2}\right)}\right] \text {, }
\end{aligned}
$$

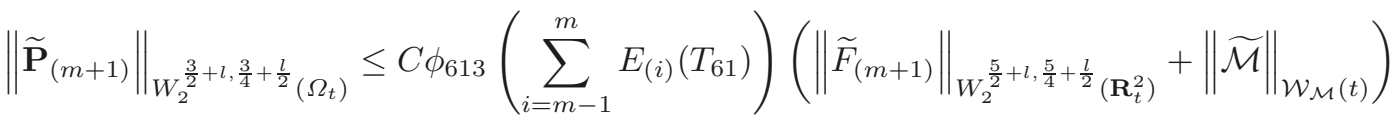

$$
\begin{aligned}
& +\left(\epsilon+C_{\epsilon} t\right) \phi_{614}\left(\sum_{m}^{i=m+1} E_{(i)}(t)\right) \widetilde{E}_{(m)}(t), \\
& \left\|\widetilde{F}_{(m+1)}\right\|_{W_{2}^{\frac{5}{2}+l, \frac{5}{4}+\frac{l}{2}}\left(\mathbf{R}_{t}^{2}\right)} \leq C \phi_{615}\left(E_{(m)}(t)\right)\left\|\tilde{\mathbf{u}}_{(m+1)}^{\prime}\right\|_{W_{2}^{3+l, \frac{3+l}{2}}\left(\Omega_{t}\right)} \\
& +\left(\epsilon+C_{\epsilon} t\right) \phi_{616}\left(E_{(m-1)}(t)+E_{(m)}(t)\right) \widetilde{E}_{(m)}(t) .
\end{aligned}
$$

By manipulating (6.11)-(6.13) as we have done for (6.7)-(6.9), we arrive at the inequality

$$
\widetilde{E}_{(m+1)}(t) \leq\left(\epsilon+C_{\epsilon} t\right) \phi_{618}\left(E_{(m-1)}\left(T_{61}\right)+E_{(m)}\left(T_{61}\right)+E_{(m+1)}\left(T_{61}\right)\right) \widetilde{E}_{(m)}(t)
$$

for any $t \in\left(0, T_{61}\right]$. Take $\epsilon$ small enough again so that $\epsilon \phi_{618}(3 M)<1$ holds, and then $T_{62} \in\left(0, T_{61}\right]$ so that $C_{\epsilon} T_{62} \phi_{618}(3 M)<1-\epsilon \phi_{618}(3 M)$ holds. For these $\epsilon$ and $T_{62}$, we obtain

$$
\widetilde{E}_{(m+1)}(t) \leq r \widetilde{E}_{(m)}(t), \quad r=\left(\epsilon+C_{\epsilon} T_{62}\right) \phi_{618}(3 M) \in(0,1) .
$$

Then we can verify that $\left\{\left(\mathcal{U}_{(m)}, \mathbf{P}_{(m)}, F_{(m)}\right)\right\}_{m=0}^{\infty}$ is a Cauchy sequence in $\mathcal{W}\left(T_{62}\right)$. Therefore the limit

$$
(\mathcal{U}, \mathbf{P}, F) \equiv \lim _{m \rightarrow \infty}\left(\mathcal{U}_{(m)}, \mathbf{P}_{(m)}, F_{(m)}\right)
$$

exists in $\mathcal{W}\left(T_{62}\right)$, which is our desired solution.

\subsection{Boundedness of temperature and salinity}

Finally we shall show that $0<\underline{T}_{0} / 2 \leq \tilde{T}(y, t)<\infty$ and $0<\underline{S}_{0} / 2 \leq \tilde{S}(y, t)<\infty$ hold by taking the time interval small enough again. Indeed, since $\tilde{T}^{\prime} \equiv \tilde{T}-T_{0} \in W_{2}^{2+l, \frac{2+l}{2}}\left(\Omega_{T_{62}}\right)$ and $\left.\tilde{T}^{\prime}\right|_{t=0}=0$, we have for $t \in\left(0, T_{62}\right]$

$$
\tilde{T}(y, t) \geq T_{0}(y)-\left|\tilde{T}(y, t)-T_{0}(y)\right| \geq \underline{T}_{0}-t^{\gamma} \sup _{y \in \Omega}\left|\tilde{T}^{\prime}(y, t)\right|_{t}^{(\gamma)}
$$

with exponent $0<\gamma<\frac{l}{2}-\frac{1}{4}$. Since Sobolev embedding theorem implies $\sup _{y \in \Omega}\left|\tilde{T}^{\prime}(y, t)\right|_{t}^{(\gamma)} \leq\left\|\tilde{T}^{\prime}\right\|_{W_{2}^{2+l,} \frac{2+l}{2}}{ }_{\left(\Omega_{T_{62}}\right)}$, with this $\gamma$, if we take

$$
T_{63} \equiv T_{62} \wedge\left(\frac{\underline{T}_{0}}{2\left\|\tilde{T}^{\prime}\right\|_{W_{2}^{2+l, \frac{2+l}{2}}\left(\Omega_{T_{62}}\right)}}\right)^{\frac{1}{\gamma}}
$$

then we have $\underline{T}_{0} / 2 \leq \tilde{T}(y, t)<\infty$ on $\left[0, T_{63}\right]$. A similar argument holds for $\tilde{S}$, and both $\underline{T}_{0} / 2 \leq \tilde{T}(y, t)<\infty$ and $\underline{S}_{0} / 2 \leq \tilde{S}(y, t)<\infty$ hold on the time interval $\left(0, T_{63}\right]$ (Take $T_{63}$ small enough again, if necessary). This provides the desired result. Uniqueness of the solution can be proved by virtue of an analogous inequality to (6.14). This completes the proof of the main theorem. 


\section{ApPEndix A. List of FUNCTION SPACES}

TABLE 1. Function spaces.

\begin{tabular}{|l|l|}
\hline $\mathcal{W}_{0}$ & $\bar{W}_{2, c}^{3+l}(\Omega) \times\left(\bar{W}_{2}^{2+l}(\Omega)\right)^{2}$ \\
\hline $\mathcal{W}\left(T^{*}\right)$ & $W_{2}^{3+l, \frac{3+l}{2}}\left(\Omega_{T_{1}}\right) \times\left(\bar{W}_{2}^{3+l, \frac{3+l}{2}}\left(\Omega_{T_{1}}\right)\right)^{2} \times W_{2}^{\frac{3}{2}+l, \frac{3}{4}+\frac{l}{2}}\left(\Omega_{T_{1}}\right) \times W_{2}^{\frac{5}{2}+l, \frac{5}{4}+\frac{l}{2}}\left(\mathbf{R}_{T_{1}}^{2}\right)$ \\
\hline $\mathcal{W}_{1}\left(T_{1}\right)$ & $\left(W_{2}^{1+l, \frac{1+l}{2}}\left(\Omega_{T_{1}}\right)\right)^{3}$ \\
\hline $\mathcal{W}_{2}\left(T_{1}\right)$ & $\left(W_{2}^{\frac{3}{2}+l, \frac{3}{4}+\frac{l}{2}}\left(\Gamma_{T_{1}}\right)\right)^{3}$ \\
\hline $\mathcal{W}_{3}\left(T_{1}\right)$ & $\left(W_{2}^{\frac{3}{2}+l, \frac{3}{4}+\frac{l}{2}}\left(\Gamma_{b T_{1}}\right)\right)^{3}$ \\
\hline $\mathcal{W}_{\mathcal{H}}\left(T_{1}\right)$ & $\prod_{i=1}^{3} \mathcal{W}_{i}\left(T_{1}\right)$ \\
\hline $\mathcal{W}_{\mathcal{U}}\left(T_{1}\right)$ & $W_{2}^{3+l, \frac{3+l}{2}}\left(\Omega_{T_{1}}\right) \times \bar{W}_{2}^{3+l, \frac{3+l}{2}}\left(\Omega_{T_{1}}\right) \times \bar{W}_{2}^{3+l, \frac{3+l}{2}}\left(\Omega_{T_{1}}\right)$ \\
\hline $\mathcal{W}_{\mathcal{U}}^{\prime}\left(T_{1}\right)$ & $\left(W_{2}^{3+l, \frac{3+l}{2}}\left(\Omega_{T_{1}}\right)\right)^{3}$ \\
\hline $\mathcal{W}^{\prime}\left(T_{1}\right)$ & $\mathcal{W}_{\mathcal{U}}^{\prime}\left(T_{1}\right) \times W_{2}^{\frac{3}{2}+l, \frac{3}{4}+\frac{l}{2}}\left(\Omega_{T_{1}}\right) \times W_{2}^{\frac{5}{2}+l, \frac{5}{4}+\frac{l}{2}}\left(\mathbf{R}_{T_{1}}^{2}\right)$ \\
\hline $\mathcal{W}_{\mathcal{M}\left(T_{1}\right)}\left(\bar{W}_{2}^{3+l, \frac{3+l}{2}}\left(\Omega_{T_{1}}\right)\right)^{2}$ \\
\hline $\mathcal{W}_{1(+)}\left(T_{1}\right)$ & $\left(W_{2}^{1+l, \frac{1+l}{2}}\left(\mathbf{R}_{(+) T_{1}}^{3}\right)\right)^{3}$ \\
\hline $\mathcal{W}_{2(+)}\left(T_{1}\right)$ & $\left(W_{2}^{\frac{3}{2}+l, \frac{3}{4}+\frac{l}{2}}\left(\mathbf{R}_{T_{1}}^{2}\right)\right)^{3}$ \\
\hline
\end{tabular}

\section{REFERENCES}

[1] P. Azerad and F. Guillén-González, Mathematical justification of the hydrostatic approximation in the primitive equations of geophysical fluid dynamics. SIAM J. Math. Anal. 33 (2001) 847-859.

[2] J.T. Beale, Large-time regularity of viscous surface waves. Arch. Rat. Mech. Anal. 84 (1984) 307-352.

[3] F.J. Beron-Vera, J. Ochoa and P. Ripa, A note on boundary conditions for salt and freshwater balances. Ocean Modelling 1 (1999) 111-118.

[4] V. Bjerknes, Das problem von der wettervorhersage, betrachtet vom standpunkt der mechanik und der physik. Meteor. Z. 21 (1904) 1-7.

[5] O. Besson and M.R. Laydi, Some estimates for the anisotropic Navier-Stokes equations and for the hydrostatic approximation. RAIRO: M2AN-Mod. Math. Anal. Numér. 26 (1992) 855-865.

[6] R. Bleck and D.B. Boudra, Wind-driven spin-up eddy resolving ocean models formulated in isopycnic and isobaric coordinates. J. Geophys. Res. 91 (1986) 7611-7621.

[7] A.F. Blumberg and G.L. Mellor, A description of a three-dimensional coastal ocean circulation model, in vol. 4 ThreeDimensional Coastal Ocean Models. Edited by N. Heaps. American Geophysical Union (1987) 1-16.

[8] D. Bresch, A. Kazhikhov and J. Lemoine, On the two-dimensional hydrostatic Navier-Stokes equations. SIAM. J. Math. Anal. 36 (2004) 796-814.

[9] K. Bryan, A numerical method for the study of the circulation of the world ocean. J. Comput. Phys. 135 (1969) 154-169.

[10] K. Bryan and M.D. Cox, An approximate equation of state for numerical models of ocean circulation. J. Phys. Oceanogr. 2 (1972) 510-514.

[11] C. Cao and E.S. Titi, Global well-posedness of the three-dimensional viscous primitive equations of large scale ocean and atmosphere dynamics. Ann. Math. 166 (2007) 245-267. 
[12] M.D. Cox, A primitive equation, three-dimensional model of the ocean. GFDL Ocean Group Technical Report 1 (1984).

[13] W.P. Crowley, A global numerical ocean model. J. Comput. Phys. 3 (1968) 111-147.

[14] W.P. Crowley, A numerical model for viscous, free-surface, barotropic wind driven ocean circulations. J. Comput. Phys. 5 (1970) 139-168.

[15] J.K. Dukowicz and R.D. Smith, Implicit free-surface method for the Bryan-Cox-Semtner ocean model. J. Geophys. Research 99 (1994) 7991-8014.

[16] S.M. Griffies et al., Developments in ocean climate modelling. Ocean Modelling 2 (2000) 123-192.

[17] S.M. Griffies, R.C. Pacanowski, M. Schmidt and V. Balaji, Tracer concentration with an explicit free surface method for $z$-coordinate ocean models. Mon. Wea. Rev. 5 (2001) 1081-1098.

[18] S.M. Griffies, Fundamentals of Ocean Climate Models. Princeton University Press, Princeton (2004).

[19] F. Guillén-González and M.A. Rodríguez-Bellido, On the strong solutions of the primitive equations in 2D domains. Nonlinear Anal. 50 (2002) 621-646.

[20] F. Guillén-González and M.A. Rodríguez-Bellido, A review on the improved regularity for the primitive equations, in Regularity and other aspects of the Navier-Stokes equations, edited by P. Mucha, P. Penel, M. Wiegner and W. Zajaczkowski. In vol. 70 of Banach Center (2005) 85-103.

[21] F. Guillén-González, N. Masmoudi and M.A. Rodríguez-Bellido, Anisotropic estimates and strong solutions of the Primitive Equations. Differ. Int. Eq. 14 (2001) 1381-1408.

[22] H. Hasumi, CCSR Ocean Component Model (COCO) Version 2.1, CCSR Report No. 13 (2000).

[23] H. Honda, Small-time Existence of a Strong Solution of Primitive Equations for the Ocean and the Atmosphere. Ph.D. thesis, Keio University, Japan (2011).

[24] H. Honda and A. Tani, Small-time existence of a strong solution of primitive equations of the coupled atmosphere and the ocean. Sūrikaisekikenkyūsho Kōkyūroku 1631 (2009) 12-33.

[25] H. Honda and A. Tani, Small-time existence of a strong solution of primitive equations for the atmosphere. Adv. Math. Sci. Appl. 20 (2010) 547-583.

[26] H. Honda and A. Tani, Small-time existence of a strong solution of primitive equations for the ocean. Tokyo J. Math. 35 (2012) 97-138.

[27] C. Hu, Asymptotic analysis of the primitive equations under the small depth assumption. Nonlin. Anal. 61 (2005) 425-460.

[28] C. Hu, R. Temam and M. Ziane, The primitive equations on the large scale ocean under small depth hypothesis. Discrete Contin. Dyn. Syst. 9 (2003) 97-131.

[29] J.H. Jones, Vertical mixing in the Equatorial Undercurrent. J. Phys. Oceanogr. 3 (1973) $286-296$.

[30] P.D. Killworth, D. Stainforth, D.J. Webb and S.M. Peterson, The Development of a Free-Surface Bryan-Cox-Semtner Ocean Model. J. Phys. Oceanogr. 21 (1991) 1333-1348.

[31] E.B. Kraus and J.S. Turner, A one-dimensional model of the seasonal thermocline. Tellus 19 (1967) 98-106.

[32] O.A. Ladyženskaja, V.A. Solonnikov and N.N. Ural'ceva, Linear and Quasi-linear Equations of Parabolic Type. In vol. 23 of Transl. Math. Monogr. American Mathematical Society (1968).

[33] J.L. Lions, R. Temam and S. Wang, New formulations of the primitive equations of atmosphere and applications. Nonlin. $\mathbf{5}$ (1992) 237-288.

[34] J.L. Lions, R. Temam and S. Wang, On the equations of the large-scale-ocean. Nonlin. 5 (1992) 1007-1053.

[35] J.L. Lions, R. Temam and S. Wang, Models for the coupled atmosphere and ocean. Comput. Mech. Adv. 1 (1993) 5-54.

[36] J.L. Lions, R. Temam and S. Wang, Numerical analysis of the coupled atmosphere and ocean models. Comput. Mech. Adv. 1 (1993) $55-120$.

[37] J.L. Lions, R. Temam and S. Wang, Problemes a frontiere libre pour les modeles couples de l'Ocean et de l'Atmosphere. C. R. Acad. Sci. Paris 318 (1994) 1165-1171.

[38] J.L. Lions, R. Temam and S. Wang, Mathematical theory for the coupled atmosphere-ocean models. J. Math. Pures Appl. 74 (1995) 105-163.

[39] J.L. Lions, R. Temam and S. Wang, On mathematical problems for the primitive equations of the ocean: the mesoscale midlatitude case. Nonlinear Anal. 40 (2000) 439-482.

[40] https://www.pik-potsdam.de/research/earth-system-analysis/models/climber/climber3/ocean.html

[41] http://www.gfdl.noaa.gov/ocean-model

[42] R.C. Pacanowski and S.H. Philander, Parameterization of vertical mixing in numerical models of tropical oceans. J. Phys. Oceanogr. 11 (1981) 1443-1451.

[43] L.F. Richardson, Weather Prediction by Numerical Process. Cambridge University Press (1922).

[44] A.J. Semtner, A general circulation model for the world ocean. UCLA Dept. Meteorol. Tech. Report 8 (1974) 99-120.

[45] V.A. Solonnikov, Solvability of a problem of evolution of a viscous incompressible fluid bounded by a free surface in a finite time interval. St. Peters. Math. J. 3 (1992) 189-220.

[46] V.A. Solonnikov and A. Tani, Free boundary problem for a compressible flow with a surface tension, in Constantin Carathéodory: International Tribute, edited by Th. M. Rassias. World Scientific Publ. Co. (1991) 1270-1309.

[47] N. Tanaka, Two-phase free boundary problem for viscous incompressible thermo-capillary convection. Japan J. Math. 21 (1995) 1-42.

[48] V.A. Solonnikov, On Boundary Value Problems to the Linear Parabolic Systems of Differential Equations of General Form (Russian), in Trudy. Mat. Inst. Steklov. 83 (1965) 3-163. English Transl. Proc. Steklov. Math. Inst. 83 (1965) 1-184. 
[49] N. Tanaka and A. Tani, Large-time existence of surface waves in incompressible viscous fluids with or without surface tension. Arch. Rat. Mech. Anal. 130 (1995) 303-314.

[50] N. Tanaka and A. Tani, Surface waves for a compressible viscous fluid. J. Math. Fluid Mech. 5 (2003) $303-363$.

[51] R. Temam and M. Ziane, Some mathematical problems in geophysical fluid dynamics, in Handb. Math. Fluid Dyn., vol. III. Edited by S.J. Friedlander, D. Serre. North-Holland (2004) 535-657.

[52] UNESCO, 1981: Tenth report of the Joint Panel on Oceanographic Tables and Standards. Unesco Technical papers in marine science, No. 36, 25pp. Sidney, B.C. (1980).

[53] W.M. Washington and C.L. Parkinson, An Introduction to Three-Dimensional Climate Modeling. Oxford University Press (1986).

[54] J. Wloka, Partielle Differentialgleichungen. B.G. Teubner (1982).

[55] M. Ziane, Regularity results for Stokes type systems. Appl. Anal. 58 (1995) 263-292.

[56] M. Ziane, Regularity results for the stationary primitive equations of atmosphere and the ocean. Nonlinear Anal. 28 (1997) $289-313$. 